\title{
ON GENERALIZED QUATERNIONS
}

\section{BY \\ GORDON PALL}

1. Introduction. In this article we shall present a basic investigation of the arithmetics of generalized quaternions, as they arise naturally out of the study of integral ternary quadratic forms. It was, indeed, out of the theory of quadratic forms, that (perhaps unnoticed) generalized quaternions first originated, in a form suitable for use in arithmetic. Thus, in 1854, Hermite [6] ( $\left.{ }^{1}\right)$ obtained a general expression for the automorphs of a ternary quadratic form. He found it simpler to express the automorphs, not of $f=\sum a_{\alpha \beta} x_{\alpha} x_{\beta}$ $\left(a_{\alpha \beta}=a_{\beta \alpha} ; \alpha, \beta=1,2,3\right)$, but of its adjoint adj $f=\sum A_{\alpha \beta} x_{\alpha} x_{\beta}$, where the $A_{\alpha \beta}$ are the cofactors of the $a_{\alpha \beta}$. (As the automorphs of $f$ are the transpose of those of $\operatorname{adj} f$, this involves no loss.) Hermite expressed the automorphs of adj $f$ (cf. \$2) by means of four parameters, say $t_{0}, t_{1}, t_{2}, t_{3}$, subject to the condition $t_{0}{ }^{2}+\sum A_{\alpha \beta} t_{\alpha} t_{\beta}=1$. Now a product of two automorphs is, from their very nature, again an automorph. He mite found that the product of the two automorphs corresponding to the parameters $u_{i}$ and $t_{i}$ is given by $v_{i}$, where

$$
\begin{gathered}
v_{0}=u_{0} t_{0}-\sum A_{\alpha \beta} u_{\alpha} t_{\beta}, \\
v_{\alpha}=u_{0} t_{\alpha}+u_{\alpha} t_{0}+\left(u_{2} t_{3}-u_{3} t_{2}\right) a_{1 \alpha}+\left(u_{3} t_{1}-u_{1} t_{3}\right) a_{2 \alpha}+\left(u_{1} t_{2}-u_{2} t_{1}\right) a_{3 \alpha} .
\end{gathered}
$$

Further, under (1), there holds the "composition identity"

$$
\left(u_{0}^{2}+\sum A_{\alpha \beta} u_{\alpha} u_{\beta}\right)\left(t_{0}^{2}+\sum A_{\alpha \beta} t_{\alpha} t_{\beta}\right)=\left(v_{0}^{2}+\sum A_{\alpha \beta} v_{\alpha} v_{\beta}\right) .
$$

This formula for multiplying the quaternion $\left(u_{0}, u_{1}, u_{2}, u_{3}\right)$ by $\left(t_{0}, t_{1}, t_{2}, t_{3}\right)$ to obtain the product quaternion $\left(v_{0}, v_{1}, v_{2}, v_{3}\right)$ is the essence of a quite general, linear, associative, quaternion algebra. U. V. Linnik [11] proposed to call the elements of this algebra hermitions. The author developed some factorization properties of these algebras in 1938 [12], in the special case where the matrix $\left(a_{\alpha \beta}\right)$ is integral. These results are perfected in the present article, and extended to the case where the form $f=\sum a_{\alpha \beta} x_{\alpha} x_{\beta}$ is integral, that is, the $a_{\alpha \alpha}$ and $2 a_{\alpha \beta}$ are integers.

With each form $f$, where the $a_{\alpha \beta}$ are rational numbers and $\left|a_{\alpha \beta}\right| \neq 0$, is associated the quaternion algebra defined by (1). The elements of this algebra can be written as $u=u_{0}+i_{1} u_{1}+i_{2} u_{2}+i_{3} u_{3}$, where the $u_{i}$ are rational numbers and the $i_{\alpha}$ satisfy the multiplication table of $\$ 2(1)$. If we apply the rational transformation $U=\left(u_{\alpha \beta}\right)$ to $\left(a_{\alpha \beta}\right)$, then by Theorem 1 , the basal elements $i_{\alpha}$ are replaced by $k_{\alpha}=U_{1 \alpha} i_{1}+U_{2 \alpha} i_{2}+U_{3 \alpha} i_{3}$, where the $U_{\alpha \beta}$ are the cofactors of

Presented to the Society, November 24, 1945; received by the editors July 26, 1945.

(') Numbers in brackets refer to the Bibliography at the end of the paper. 
the $u_{\alpha \beta}$. Thus the algebras associated with $\left(a_{\alpha \beta}\right)$ and $U^{\prime}\left(a_{\alpha \beta}\right) U$ are rationally equivalent. Indeed, the correspondence between elements $u_{0}+\sum u_{\alpha} i_{\alpha}$ and $v_{0}+\sum v_{\alpha} k_{\alpha}$ becomes actual equality under the transformation $v_{0}=u_{0}, u_{\alpha}=U_{\alpha 1} v_{1}$ $+U_{\alpha 2} v_{2}+U_{\alpha 3} v_{3}$.

The system of Hamiltonian quaternions (for which $i_{\alpha}{ }^{2}=-1, i_{2} i_{3}=i_{1}$ $=-i_{3} i_{2}$, and so on) is associated with $f_{1}=x_{1}{ }^{2}+x_{2}{ }^{2}+x_{3}{ }^{2}$, or with the identity matrix.

With every integral form $f$ is associated a system $\sum(f)$ of integral quaternions, consisting of the quantities $t=t_{0}^{\prime}+\sum i_{\alpha} t_{\alpha}$, where $t_{0}^{\prime}=t_{0}+2^{-1} \sum \epsilon_{\alpha} t_{\alpha}$, and $t_{0}, t_{1}, t_{2}, t_{3}$ are rational integers (\$3). Here the $\epsilon_{\alpha}$ have the values 0 or 1 in accordance with $\S 3(1)$. The sum, difference, and product of two elements of $\sum(f)$ are in $\sum(f)$. The trace $2 t_{0}^{\prime}$ and the norm $\left(t_{0}+2^{-1} \sum \epsilon_{\alpha} t_{\alpha}\right)^{2}+\sum A_{\alpha \beta} t_{\alpha} t_{\beta}$ are rational integers. The quantities 1 and $j_{\alpha}=i_{\alpha}+\epsilon_{\alpha} / 2(\alpha=1,2,3)$ form a basis of $\sum(f)$. If we apply a unimodular transformation $U$ to $f$ to obtain $g$, the systems $\sum(f)$ and $\sum(g)$ are isomorphic, and the trace and norm of each element are invariant. If $U$ is integral, but $|U|>1, \sum(g)$ is a subset of $\sum(f)$. The system $\sum(f)$ is maximal in the sense of Dickson, if and only if the form adj $f$ cannot be derived by an integral transformation from any form adj $g$, where $g$ is also integral (cf. \$3).

If $f=f_{1}$, then $\sum(f)$ consists of the Lipschitz integral quaternions

$$
t_{0}+i_{1} t_{1}+i_{2} t_{2}+i_{3} t_{3}
$$

the $t_{i}$ rational integers, the $i_{\alpha}$ the Hamiltonian units. This system is not maximal. For, if $g_{1}=x_{1}{ }^{2}+x_{2}{ }^{2}+x_{3}{ }^{2}+x_{2} x_{3}+x_{3} x_{1}+x_{1} x_{2}$, then adj $g_{1}=\left(3 x_{1}{ }^{2}+3 x_{2}{ }^{2}+3 x_{3}{ }^{2}\right.$ $\left.-2 x_{2} x_{3}-2 x_{3} x_{1}-2 x_{1} x_{2}\right) / 4$, and $\operatorname{adj} f_{1}=y_{1}{ }^{2}+y_{2}{ }^{2}+y_{3}{ }^{2}$ is obtained from adj $g_{1}$ by the transformation $x_{1}=y_{2}+y_{3}, x_{2}=y_{1}+y_{3}, x_{3}=y_{1}+y_{2}$ of determinant 2 .

Any system $\sum(f)$ can be put (cf. $\left.\$ 11\right)$ into other forms by applying rational transformations, and expressing the conditions on the coefficients of elements in the resulting algebras which correspond to the integrality of the elements of $\sum(f)$. For example, since the diagonal multiplication table for $f_{1}$ is simpler than that for $g_{1}$ (in the preceding paragraph), we may seek a set of elements in the quaternion algebra for $f_{1}$ arithmetically equivalent to the set $\sum\left(g_{1}\right)$. With an eye on the identity

$$
\operatorname{adj} g_{1}=\left(x_{2}+x_{3}-x_{1}\right)^{2} / 4+\left(x_{3}+x_{1}-x_{2}\right)^{2} / 4+\left(x_{1}+x_{2}-x_{3}\right)^{2} / 4
$$

and noting that $\epsilon_{1}=\epsilon_{2}=\epsilon_{3}=1$ for $g_{1}$, we set

$$
\begin{array}{ll}
y_{0}=2 x_{0}+x_{1}+x_{2}+x_{3}, & y_{1}=x_{2}+x_{3}-x_{1}, \\
y_{2}=x_{3}+x_{1}-x_{2}, & y_{3}=x_{1}+x_{2}-x_{3},
\end{array}
$$

whence the norm-form $\left(x_{0}+2^{-1} \sum \epsilon_{\alpha} x_{\alpha}\right)^{2}+\sum A_{\alpha \beta} x_{\alpha} x_{\beta}$ becomes $\left(y_{0}{ }^{2}+y_{1}{ }^{2}+y_{2}{ }^{2}\right.$ $\left.+y_{3}{ }^{2}\right) / 4$. On solving (5) for the $x_{i}$ we obtain $2 x_{1}=y_{2}+y_{3}, 2 x_{2}=y_{3}+y_{1}, 2 x_{3}=$ $y_{1}+y_{2}, 2 x_{0}=y_{0}-y_{1}-y_{2}-y_{3}$, whence the integrality condition that $x_{0}+\sum j_{\alpha} x_{\alpha}$ 
have integral coordinates is equivalent to the condition, that $y_{0}, y_{1}, y_{2}, y_{3}$ are integers satisfying

$$
y_{0} \equiv y_{1} \equiv y_{2} \equiv y_{3}(\bmod 2) \text {; }
$$

while the corresponding integral quaternions are given by

$$
\left(y_{0}+i_{1} y_{1}+i_{2} y_{2}+i_{3} y_{3}\right) / 2 \text {, }
$$

the $i_{\alpha}$ being the Hamiltonian units. Thus this system of integral quaternions, given by A. Hurwitz, is isomorphic with $\sum\left(g_{1}\right)$. The form $g_{1}$, we should observe, is the reduced, positive-definite, integral ternary of least determinant $(=1 / 2)$.

The much debated question of whether one should use the Hurwitz system rather than that of Lipschitz is thus seen from the point of view of quadratic form theory to amount to this, whether one should confine attention to fundamental forms (not derivable from integral forms of a smaller determinant) or not. The form $f_{1}=x_{1}{ }^{2}+x_{2}{ }^{2}+x_{3}{ }^{2}$ is, to the worker with quadratic forms, just as important as the form $g_{1}$, even though the form adj $f_{1}$ may not be fundamental. Similarly, from the standpoint of quadratic forms, nonmaximal systems of integral quaternions are just as important as maximal systems, even though they may not be as simple (for, if adj $g$ is carried into adj $f$ by an integral transformation of determinant $k$, the primes dividing $k$ will play an exceptional role in $\sum(f)$ ).

In this article we shall not confine attention to maximal systems. We shall make no restriction on $f$, for arithmetical applications, except that it be integral. However, we shall sometimes be compelled to restrict the norms of certain quaternions, and it will usually be seen that these restrictions are vacuous when the system is maximal.

Out of personal experience with Hurwitz or Lipschitz quaternions, the author may say that it makes no essential difference which one uses. Using Lipschitz quaternions one must sometimes restrict the norm to be odd, but this is counterbalanced by the simplifying fact that there are fewer units.

Interesting points to which we may call attention here are: the easily remembered multiplication table $(\$ 2(1))$, and its simple law of transformation (Theorem 1); the definition of a system of integral quaternions ( $\$ 3$ ); the theorem on the uniqueness of factorization of primitive quaternions in every system $\sum(f)(\S 5)$; the algorithm for finding factors of a given norm in $\S 6$; the exact formula (when $F$ is fundamental) for the integral automorphs of adj $f$ and the norm-form $F$, which points to the essential rightness of our definition of integral quaternion $(\$ 7)$; the determination of all systems with positivedefinite norm-forms in which factorization is always possible (Theorems 10 and 11). We may point also to Theorem 12, which states that there do not exist genera of more than one class of positive-definite norm-forms, which do not also contain classes of minimum greater than 1 ; there should surely be an easier way of proving this. 
Principal notations. $f$ denotes a ternary form of matrix $\left(a_{\alpha \beta}\right)$; the matrix of adj $f$ is $A=\left(A_{\alpha \beta}\right)$; the multiplication table of its algebra is given in $\$ 2,(1)$ or (2); the basal elements of the algebra are usually designated $1, i_{1}, i_{2}, i_{3}$; elements of the algebra, quaternions, are usually, denoted by the letters $t, u, \cdots, z$, and their coordinates indicated by subscripts. If $f$ is an integral form, a basis of the integral elements $\sum(f)$ is $1, j_{1}, j_{2}, j_{3}(\S 3) ; d=4\left|a_{\alpha \beta}\right|$; $F$ always denotes the norm-form $\left(x_{0}+\epsilon_{1} x_{1} / 2+\epsilon_{2} x_{2} / 2+\epsilon_{3} x_{3} / 2\right)^{2}+\sum A_{\alpha \beta} x_{\alpha} x_{\beta}$, where the $\epsilon_{\alpha}$ (=0 or 1 ) are chosen to make $F$ an integral form. Subscripts $\alpha$ and $\beta$ always range over $1,2,3$; subscripts $i$ and $j$ over $0,1,2,3$, unless otherwise indicated. $F, G$ denote quaternary forms; other italic capitals denote square matrices and their linear transformations; $T^{\prime}=$ transpose of matrix $T$; $\bar{t}=$ the conjugate of the quaternion $t(\$ 2)$.

We shall employ freely (without indications of proof) certain facts about the form-residues modulo $k$ to which a quadratic form can be reduced by integral transformations of determinant prime to $k$; certain standard properties of genera of quadratic forms; and the invariants $c_{p}$ of quadratic forms under rational, linear transformations. The latter invariants are due to Hasse [4], and in the ternary case to Hensel [5, p. 337]. An exposition of all these tools will be given shortly in a book by the author.

2. The Hermite quaternion algebra. The quaternion algebra pertaining to the form $f$, or symmetric matrix $\left(a_{\alpha \beta}\right)$, will now be defined. This algebra has four basal elements $1, i_{1}, i_{2}, i_{3}$ satisfying the multiplication table

$$
\begin{gathered}
i_{\alpha}{ }^{2}=-A_{\alpha \alpha} \quad(\alpha=1,2,3), \\
i_{2} i_{3}=-A_{23}+\sum a_{1 \alpha} i_{\alpha}, \quad i_{3} i_{2}=-A_{32}-\sum a_{1 \alpha} i_{\alpha},
\end{gathered}
$$

with $i_{3} i_{1}$, and so on, obtained by permuting subscripts cyclically. In matrix notations, the multiplication table can be written

(2) $\mathfrak{i i}^{\prime}=-A+K$, where $K=\left[\begin{array}{ccc}0 & \sum a_{3 \alpha} i_{\alpha} & -\sum a_{2 \alpha} i_{\alpha} \\ -\sum a_{3 \alpha} i_{\alpha} & 0 & \sum a_{1 \alpha} i_{\alpha} \\ \sum a_{2 \alpha} i_{\alpha} & -\sum a_{1 \alpha} i_{\alpha} & 0\end{array}\right]$,

where $i^{\prime}$ denotes the row vector $\left(i_{1}, i_{2}, i_{3}\right)$ and the prime indicates "transpose." The elements of the algebra have the form

$$
x=x_{0}+\sum i_{\alpha} x_{\alpha}=x_{0}+\mathfrak{i}^{\prime} \xi,
$$

where $\xi^{\prime}=\left(x_{1}, x_{2}, x_{3}\right)$; and the $x_{i}$ may range over some field containing the coefficients $a_{\alpha \beta}$.

It is sometimes more convenient to speak of the $i_{\alpha}$ as pertaining to the matrix $\left(A_{\alpha \beta}\right)$. Note that $\left(A_{\alpha \beta}\right)$ is the adjoint also of $\left(-a_{\alpha \beta}\right)$, and that the corresponding multiplication table is obtained by changing the $i_{\alpha}$ for $\left(a_{\alpha \beta}\right)$ to their negatives. 
We can easily verify that if $u=u_{0}+\sum i_{\alpha} u_{\alpha}$ and $t=t_{0}+\sum i_{\alpha} t_{\alpha}$, then $u t=v$ is given by Hermite's formulae (1) of $\$ 1$.

If $T$ is any nonsingular matrix of order 3 ,

$$
x_{0}+\mathfrak{i}^{\prime} \xi=x_{0}+\mathfrak{l}^{\prime} \eta, \quad \text { where } \xi=T \eta, \quad \mathfrak{l}^{\prime}=\mathfrak{i}^{\prime} T .
$$

By (2), $\mathfrak{l f}^{\prime}=T^{\prime} \mathfrak{i i} T=-T^{\prime} A T+T^{\prime} K T$. This suggests the following theorem.

THEOREM 1. Let $U$ denote a matrix of order 3 and nonzero determinant $\lambda$. If $\left(a_{\alpha \beta}\right)$ is replaced by $\left(b_{\alpha \beta}\right)=U^{\prime}\left(a_{\alpha \beta}\right) U$, whence $A$ is replaced by $B=T^{\prime} A T$ (where $\left.T^{\prime} U=U T^{\prime}=\lambda I\right)$, then the basal elements $k_{\alpha}$ pertaining to $B$ are related to the $i_{\alpha}$ of $A$ by the linear transformation

$$
\mathfrak{l}=T^{\prime} \mathbf{i} \text {. }
$$

We shall base our proof on the interesting identity:

$$
C^{\prime}\left[\mathfrak{p}^{*}\right] C=\left[q^{*}\right], \text { where } \mathfrak{q}=D \mathfrak{p} .
$$

Here $\mathfrak{p}$ denotes any column vector, $\mathfrak{p}^{\prime}=\left(p_{1}, p_{2}, p_{3}\right)$, and $\left[\mathfrak{p}^{*}\right]$ denotes the skewsymmetric matrix formed from $\mathfrak{p}$ as follows:

$$
\left[p^{*}\right]=\left[\begin{array}{ccc}
0 & p_{3} & -p_{2} \\
-p_{3} & 0 & p_{1} \\
p_{2} & -p_{1} & 0
\end{array}\right] ;
$$

$C$ is any matrix of order 3 , and $D^{\prime}$ is the matrix of its cofactors, that is, $C D=D C=\gamma I$, where $\gamma=|C|$. Obviously, the left side of (6) is skew-symmetric. We leave the verification of (6) to the reader.

To complete the proof of Theorem 1 we must show that $T^{\prime} K T=\left[q^{*}\right]$, where $\mathfrak{q}=\left(b_{\alpha \beta}\right) \mathfrak{l}$. We have immediately,

$$
\begin{aligned}
T^{\prime} K T & =T^{\prime}\left[\mathfrak{p}^{*}\right] T, \\
& =\left[\mathfrak{q}^{*}\right], \quad \text { where } \mathfrak{p}=\left(a_{\alpha \beta}\right) \mathfrak{i}, \\
& \text { where } \mathfrak{q}=\lambda U^{\prime}\left(a_{\alpha \beta}\right) \mathfrak{i}=U^{\prime}\left(a_{\alpha \beta}\right) U T^{\prime} \mathfrak{i}=\left(b_{\alpha \beta}\right) \mathfrak{l} .
\end{aligned}
$$

The form $\sum A_{\alpha \beta} x_{\alpha} x_{\beta}$ is derivable from $x_{1}{ }^{2}+x_{2}{ }^{2}+x_{3}{ }^{2}$ by means of a complex linear transformation $\xi=T r_{i}$, hence $A=T^{\prime} T$. It follows from Theorem 1 that the basal elements $i_{\alpha}$ pertaining to $A$ are related to the Hamiltonian units, say $h_{\alpha}$ (these being usually denoted by $i, j, k$ ), by the transformation $\mathfrak{i}=T^{\prime} \mathfrak{h}$.

We define the conjugate $\bar{x}$ of $x$ to be $\bar{x}=x_{0}-i_{1} x_{1}-i_{2} x_{2}-i_{3} x_{3}$. In the case of Hamiltonian quaternions, $\bar{x} x=\sum x_{i}{ }^{2}$. Hence, by the preceding paragraph, there holds in our generalized quaternion algebra,

$$
\bar{x} x=x \bar{x}=x_{0}^{2}+\xi^{\prime} A \xi=x_{0}^{2}+\sum A_{\alpha \beta} x_{\alpha} x_{\beta} .
$$

We call this the norm of $x$ and denote it by $N x$. It will be seen from (1) and the distributivity of multiplication that the conjugate of $\left(u_{0}+\sum i_{\alpha} u_{\alpha}\right)\left(t_{0}+\sum i_{\alpha} t_{\alpha}\right)$ is $\left(t_{0}-\sum i_{\alpha} t_{\alpha}\right)\left(u_{0}-\sum i_{\alpha} u_{\alpha}\right)$, that is

$$
\overline{u t}=\bar{t} \cdot \bar{u} .
$$


Hence if $v=u t$, then $\bar{v}=\bar{t} \bar{u}, v \bar{v}=u t \bar{t} \bar{u}=u \bar{u} \cdot t \bar{t}$, or $N v=N u \cdot N t$. This is of course Hermite's identity (2) of $\$ 1$.

The correspondence in (4) replaces each quaternion $x$ by an equal quaternion, expressed in terms of a new basis. This correspondence is preserved under addition and, by Theorem 1, under multiplication. The "real part" $x_{0}$ of $x$, and the norm, are invariant.

We call $x$ pure if $\bar{x}=-x$, whence $2 x_{0}=0$. If $x$ is pure and $t$ is any quaternion, then $y=\bar{t} x t$ is also pure, and $N y=N x(N t)^{2}$. Here $N x$ is given by the ternary form $\sum A_{\alpha \beta} x_{\alpha} x_{\beta}$. Thus if $N t=1$; the linear transformation expressing the $y_{\alpha}$ in terms of the $x_{\beta}$, obtained on expanding $y=\bar{t} x t$, is an automorph of the form $\sum A_{\alpha \beta} x_{\alpha} x_{\beta}$. It is of determinant +1 , and will be found to coincide with the general expression of Hermite, mentioned in the Introduction.

Indeed, we easily prove that $y=\bar{t} x t$ where $N t=1$ gives the most general automorph of determinant +1 of any form $A=\left(A_{\alpha \beta}\right)$, provided it is true for one particular form, say for the identity matrix $I$, or the matrix of $x_{1} x_{3}-x_{2}{ }^{2}$ (a relatively easy case [1, pp. 22-23]). For, if $E$ denotes the general automorph of determinant +1 of (say) $I$, and $T$ is a particular transformation of $I$ into $A$, then an obvious argument shows that $T^{-1} E T$ is the general automorph of determinant +1 of $A$. Let $t$ denote the $I$-quaternion for which $y=\bar{t} x t$ expands into $\eta=E \xi$. Consider the corresponding equation in $A$-quaternions $y=\bar{x} x t$. Then the $I$-coordinates of $y$ are those of the vector $T \eta$, and those of $x$ are those of $T \xi$; cf. (4). Hence the $A$-equation $y=\bar{t} x t$ is equivalent to the linear transformation $T \eta=E(T \xi)$, or $\eta=\left(T^{-1} E T\right) \xi$. It therefore yields every automorph of determinant +1 of $A$.

LEMMA 1. Let $\left(a_{\alpha \beta}\right)$ be a rational, symmetric matrix of order $3,\left|a_{\alpha \beta}\right| \neq 0$. Let $1, k_{1}, k_{2}, k_{3}$ be linearly independent quaternions in the algebra of $\left(a_{\alpha \beta}\right)$ satisfying the same multiplication table as the basal elements $1, i_{1}, i_{2}, i_{3}$. Then there exists a quaternion $q$ in the algebra and $a$ sign $\sigma= \pm 1$ such that

$$
\sigma k_{1}=q i_{1} q^{-1}, \quad \sigma k_{2}=q i_{2} q^{-1}, \quad \sigma k_{3}=q i_{3} q^{-1} .
$$

For since $k_{\alpha}{ }^{2}$ is real and $1, k_{1}, k_{2}, k_{3}$ are linearly independent, $k_{\alpha}$ is pure and we can find a nonsingular rational matrix $T$ such that $\mathfrak{l}=T^{\prime} \mathfrak{i}$ (cf. (5)). Hence the multiplication table of the $k_{\alpha}$ is that connected with the form $T^{\prime} A T$. By hypothesis, $T^{\prime} A T=A$, or $T$ is a rational automorph of $A$. Choose the sign of $T$ and adjust $\sigma$ so that $|T|=+1$. Then there exists a rational quaternion $t$ such that the equation $y=t x t^{-1}$ is equivalent to the matrix equation $\eta=T \xi$. If $x=i_{1}$, then $\xi^{\prime}=(1,0,0)$, and $\eta^{\prime}=\xi^{\prime} T^{\prime}$, whence $\eta^{\prime}$ is the first row of $T^{\prime}$; thus the coordinates of $\eta^{\prime}$ are the $i$-coordinates of $k_{1}$, that is $k_{1}=t i_{1} t^{-1}$. Similarly, $k_{2}=t i_{2} t^{-1}, k_{3}=t i_{3} t^{-1}$.

3. Quaternion arithmetics. Let $\left(a_{\alpha \beta}\right)$ be any nonsingular symmetric matrix of order 3 , which is semi-integral. That is, $a_{11}, a_{22}, a_{33}$, and $2 a_{23}, 2 a_{31}, 2 a_{12}$ are rational integers; and the ternary form $f=\sum a_{\alpha \beta} x_{\alpha} x_{\beta}$ is an integral form. The adjoint matrix $A=\left(A_{\iota \beta}\right)$ may have some of its coefficients with denomi- 
nator 4 . It will be convenient to introduce three numbers $\epsilon_{1}, \epsilon_{2}, \epsilon_{3}$ each equal to 0 or 1 according as (respectively) $2 a_{23}, 2 a_{31}, 2 a_{12}$ is even or odd. It is easily seen that

$$
4 A \equiv\left[\begin{array}{lll}
\epsilon_{1}{ }^{2} & \epsilon_{1} \epsilon_{2} & \epsilon_{1} \epsilon_{3} \\
\epsilon_{2} \epsilon_{1} & \epsilon_{2}{ }^{2} & \epsilon_{2} \epsilon_{3} \\
\epsilon_{3} \epsilon_{1} & \epsilon_{3} \epsilon_{2} & \epsilon_{3}{ }^{2}
\end{array}\right] \equiv \epsilon \epsilon^{\prime}(\bmod 2)
$$

where $\epsilon^{\prime}$ denotes the vector $\left(\epsilon_{1}, \epsilon_{2}, \epsilon_{3}\right)$.

The Hermite "norm-form" $x_{0}{ }^{2}+\sum A_{\alpha \beta} x_{\alpha} x_{\beta}$ is not always integral for integral forms $f$. However, it is found (cf. (1)) that the form

$$
\begin{aligned}
F= & \left(x_{0}+\epsilon_{1} x_{1} / 2+\epsilon_{2} x_{2} / 2+\epsilon_{3} x_{3} / 2\right)^{2}+\sum_{\alpha, \beta=1}^{3} A_{\alpha \beta} x_{\alpha} x_{\beta} \\
= & x_{0}^{2}+\sum \epsilon_{\alpha} x_{0} x_{\alpha}+\sum\left(A_{\alpha \alpha}+\epsilon_{\alpha}{ }^{2} / 4\right) x_{\alpha}{ }^{2}+\left(2 A_{23}+\epsilon_{2} \epsilon_{3} / 2\right) x_{2} x_{3} \\
& +\left(2 A_{31}+\epsilon_{3} \epsilon_{1} / 2\right) x_{3} x_{1}+\left(2 A_{12}+\epsilon_{1} \epsilon_{2} / 2\right) x_{1} x_{2}
\end{aligned}
$$

always has integral coefficients. By replacing $x_{0}$ by $x_{0}+\sum h_{\alpha} x_{\alpha}$, where the $h_{\alpha}$ are integers, we can evidently replace the $\epsilon_{\alpha}$ by arbitrary integers of the same respective parities. The form (2) then becomes identical with a form shown by Brandt to be the most general satisfying a certain type of composition identity. We shall refer to (2) as the Brandt norm-form or simply norm-form.

We now introduce new basal elements $j_{\alpha}$ by the equations

$$
i_{\alpha}=j_{\alpha}-\epsilon_{\alpha} / 2
$$$$
(\alpha=1,2,3),
$$

with the $\epsilon_{\alpha}$ as defined above. The elements of the algebra can be written as

$$
t=t_{0}+j_{1} t_{1}+j_{2} t_{2}+j_{3} t_{3},
$$

where the $t_{i}$ are (say) rational. We define $t$ to be an integral quaternion if the $j$-coordinates $t_{0}, t_{1}, t_{2}, t_{3}$ are rational integers. Clearly,

$$
t=t_{0}+2^{-1} \sum \epsilon_{\alpha} t_{\alpha}+\sum i_{\alpha} t_{\alpha}=t_{0}^{\prime}+\sum i_{\alpha} t_{\alpha}
$$

say, and the real part $t_{0}^{\prime}$ of an integral quaternion $t$ is in general only half an integer, while the other coordinates (always integers) are the same for both the $i_{\alpha}$ and $j_{\alpha}$.

The sum of two integral quaternions is evidently integral. Also,

$$
\begin{gathered}
j_{\alpha}{ }^{2}=\epsilon_{\alpha} j_{\alpha}-A_{\alpha \alpha}-\epsilon_{\alpha}{ }^{2} / 4 \\
j_{2} j_{3}=\left(i_{2}+\epsilon_{2} / 2\right)\left(i_{3}+\epsilon_{3} / 2\right)=-A_{23}-2^{-1} \sum a_{1 \alpha} \epsilon_{\alpha}-\epsilon_{2} \epsilon_{3} / 4 \\
+a_{11} j_{1}+\left(a_{12}+\epsilon_{3} / 2\right) j_{2}+\left(a_{13}+\epsilon_{2} / 2\right) j_{3} .
\end{gathered}
$$

Here $A_{23}+2^{-1} \sum a_{1 \alpha} \epsilon_{\alpha}+\epsilon_{2} \epsilon_{3} / 4=\left(a_{12}+\epsilon_{3} / 2\right)\left(a_{31}+\epsilon_{2} / 2\right)-a_{11}\left(a_{32}+\epsilon_{1} / 2\right)+a_{11} \epsilon_{1}$, and is always integral. Hence a product of integral quaternions is always integral. 
The norm of $t$ evidently coincides with the Brandt norm-form in the $j$-coordinates of $t$ as variables, and with the Hermite norm-form in the $i$-coordinates. If $t$ is integral, $N t$ is an integer. The same is true of $t+\bar{t}$, or $2 t_{0}^{\prime}$. Hence $t$ satisfies the algebraic equation $t^{2}-2 t_{0}^{\prime} t+N t=0$ in which the coefficients are rational integers.

A quaternion is called primitive if it is integral, and the g.c.d. of its $j$-coordinates $t_{0}, t_{1}, t_{2}, t_{3}$ is 1 ; primitive $\bmod \kappa$ if this g.c.d. is prime to $\kappa$; purelyintegral if it is pure and $t_{1}, t_{2}$, and $t_{3}$ are integers (pure meaning, even if $j$-coordinates are used, that $\left.t_{0}+2^{-1} \sum \epsilon_{\alpha} t_{\alpha}=0\right)$; purely-primitive if it is purelyintegral and the g.c.d. of $t_{1}, t_{2}, t_{3}$ is 1 . A purely-integral quaternion is therefore not integral unless $\sum \epsilon_{\alpha} t_{\alpha}$ is even.

Our set of integral elements has all the properties prescribed by L. E. Dickson [3(a), p. 141; (b), p. 154] for the integral elements of an algebra, except that in some cases our set is not maximal, and can be imbedded in a larger set of integral elements.

It can easily be shown directly, or by using some results of Brandt, or of C. G. Latimer [10], that the set $\sum(f)$ is maximal if and only if $\operatorname{adj} f$ is fundamental, in the sense that adj $f$ cannot be obtained by an integral linear transformation of determinant greater than 1 from the adjoint of an integral form. For, if $d=4\left|a_{\alpha \beta}\right|$, it is easy to prove that $\operatorname{adj} f$ is fundamental if and only if

$$
d \text { is squarefree, and } c_{p}=-1 \text { for each prime } p \text { in } d \text {. }
$$

(In view of Theorem 7 , the same result holds with $F$ in place of adj $f$.) An examination of Latimer's work will show that every maximal set of integral elements in a rational generalized quaternion algebra is associated with a ternary form satisfying (7.). It should be noted that he appears in his work to omit the condition that $c_{2}$ is -1 when $d$ is even (an essential condition if $f$ is indefinite); and that, after transforming his problem so that in effect $\epsilon_{1}=1$ and $\epsilon_{2}=\epsilon_{3}=0$, he uses as his key form the norm of $2 x_{1} i_{1}+x_{2} i_{2}+x_{3} i_{3}$ (integrality requiring that the coefficient of $i_{1}$ in a pure quaternion is even), whence his matrix $\Gamma$ is obtained from our $A$ by multiplying the first row and column by 2 .

Several writers have investigated canonical bases of (maximal) integral sets. Since our multiplication table is so easy to remember, handle, and transform, we prefer not to canonicize it any further, but rather, when we have the need, to use all the resources of quadratic form theory to obtain the most expedient form for a particular problem.

4. Integrality is preserved if $T$ is unimodular. We prove somewhat more. Suppose $T$ is an integral matrix such that $B=T^{\prime} A T$ is the adjoint of a semiintegral matrix. (This is always true if $|T|=1$.) To see that integral quaternions associated with $B$ are still integral when referred back to $A$, we need only show that

$$
\sum \epsilon_{\alpha} x_{\alpha} \equiv \sum \epsilon_{\alpha}^{*} y_{\alpha}(\bmod 2)
$$


where the $\epsilon_{\alpha}^{*}$ are the parameters 0 or 1 related to $B$; and where $\xi=T \eta$, $x_{0}+2^{-1} \sum \epsilon_{\alpha} x_{\alpha}=y_{0}+2^{-1} \sum \epsilon_{\alpha}^{*} y_{\alpha}$.

Now $4 A \equiv \epsilon \epsilon^{\prime} \bmod 2$ by $\S 3(1)$. Hence $4 B \equiv \epsilon^{*} \epsilon^{* \prime} \equiv T^{\prime} 4 A T \equiv T^{\prime} \epsilon \epsilon^{\prime} T \bmod 2$. Hence $\epsilon^{* \prime} \equiv \epsilon^{\prime} T$, and $\sum \epsilon_{\alpha} x_{\alpha}=\epsilon^{\prime} \xi=\epsilon^{\prime} T \eta \equiv \epsilon^{* \prime} \eta \equiv \sum \epsilon_{\alpha}^{*} y_{\alpha} \bmod 2$.

Note that the transformation replacing the norm-form $\left(x_{0}+2^{-1} \sum \epsilon_{\alpha} x_{\alpha}\right)^{2}$ $+\xi^{\prime} A \xi$ by the norm-form $\left(y_{0}+2^{-1} \sum \epsilon_{\alpha}^{*} y_{\alpha}\right)^{2}+\eta^{\prime} B \eta$ is the integral transformation $\xi=T \eta, x_{0}=y_{0}+\left(\epsilon^{* \prime}-\epsilon^{\prime} T\right) \eta / 2$ (see $\left.\S 8\right)$.

5. The factors of a given norm of a primitive quaternion are essentially unique. If $v=u t$ in integral quaternions, $t$ is a right-divisor, $u$ is a left-divisor, of $v$. Necessarily, $N t \mid N v$. We designate units, that is integral quaternions of norm 1 , by the letter $\theta$. The quaternions $\theta t$ are called left-associates of $t$; all, or none, are right-divisors of $v$. By definition of integral quaternion, $v$ is divisible by a rational integer $m$ if and only if each of the $j$-coordinates of $v$ is divisible by $m$. We now prove:

THEOREM 2. Let $x$ be primitive. If $N t=m$, and $t$ is a right-divisor of $x$, the only right-divisors of $x$ with norm $m$ are the left-associates $\theta t$, provided

$$
\left\{\begin{array}{l}
m \text { is not divisible by any prime } p \text { such that } p^{2} \mid d \\
\left(\text { where } d=4\left|a_{\alpha \beta}\right|\right) \text { or such that } p \| d \text { and } c_{p}=+1 .
\end{array}\right.
$$

It will be noted that the restriction (1) on $m$ is vacuous if the integral system is maximal (as we remarked in $\$ 1$ ). The proof depends on three lemmas, from which the theorem will follow, since $x+z m$ and $t$ have the same right-divisors of norm $m, t=u t_{1}$ with $N t=N t_{1}=m, N u=1, u=\theta$.

LeMma 2a. If $x \equiv y(\bmod m), x$ and $y$ have the same right-divisors of norm $m$.

Proof. If $y=x+z m$ and $x=u t$ where $N t=m$, then $y=(u+z \bar{t}) t$.

Lemma $2 \mathrm{~b}$. If $N w$ is prime to $m, x$ and $w x$ have the same right-divisors of norm $m$.

Proof. Choose $q$ so that $q N w \equiv 1(\bmod m)$. If $w x=u t, q \bar{w} w x=q \bar{w} u t \equiv x$ $(\bmod m)$.

Lemma 3. If $m$ satisfies (1), $x$ is primitive, $x=u t$, and $N t=m$, then we can choose an integral quaternion $z$ such that $N(x+z m) / m$ is prime to $m$.

Proof. Set $q=(N x) / m$. Then

$$
N(x+z m) / m=q+x \bar{z}+z \bar{x}+z \bar{z} m,
$$

and we set

$$
r=x \bar{z}+z \bar{x}=2\left(x_{0}{ }^{\prime} z_{0}^{\prime}-\sum A_{\alpha \beta} x_{\alpha} z_{\beta}\right) .
$$

We can apply to $f$ any unimodular transformation to obtain a more convenient residue, since divisibility will be invariant under such transformation. We 
interpolate here a lemma on form-residues embodying facts needed here and later.

LEMMA 4. Let $d=4\left|a_{\alpha \beta}\right|$. If $p$ is an odd prime not dividing $d$ or dividing $d$ at most once, we can assume

$$
f \equiv a_{1} x_{1}{ }^{2}+a_{2} x_{2}{ }^{2}+p^{\alpha_{3}} a_{3} x_{3}{ }^{2}\left(\bmod p^{r}\right),
$$

rarbitrary, where $a_{1}, a_{2}, a_{3}$ are prime to $p$, and $\alpha_{3}$ is 0 or 1 ; if $\alpha_{3}=1, c_{p}=\left(-a_{1} a_{2} \mid p\right)$. If $d$ is odd, $f$ is equivalent mod $2 r$ to the form

$$
f \equiv x_{1} x_{2}+a_{3} x_{3}{ }^{2},
$$

$a_{3}$ odd; and if $d \equiv 2(\bmod 4)$ and $c_{2}=-1, f$ is equivalent $\bmod 2^{r}$ to the form

$$
f \equiv x_{1}{ }^{2}+x_{1} x_{2}+x_{2}{ }^{2}+2 a_{3} x_{3}{ }^{2},
$$

$a_{3}$ odd. Form-residues for coprime moduli can be achieved simultaneously.

To continue with the proof of Lemma 3 , if $p \mid m$ and $p \nmid q$, we make $q+r$ prime to $m$ by taking $z \equiv 0\left(\bmod p^{2}\right)$. If $p|m, p| q$, and $p \nmid d$, then by (4) with $\alpha_{3}=0$ and (5),

$$
\begin{aligned}
r & \equiv 2\left(x_{0}{ }^{\prime} z_{0}^{\prime}-a_{2} a_{3} x_{1} z_{1}-a_{3} a_{1} x_{2} z_{2}-a_{1} a_{2} x_{3} z_{3}\right)\left(\bmod p^{2}\right), \text { if } p>2, \\
r & \equiv 2\left[\left(x_{0}+x_{3} / 2\right)\left(z_{0}+z_{3} / 2\right)+a_{3} x_{1} z_{2} / 2+a_{3} x_{2} z_{1} / 2+x_{3} z_{3} / 4\right] \\
& \equiv\left(2 x_{0}+x_{3}\right) z_{0}+a_{3} x_{2} z_{1}+a_{3} x_{1} z_{2}+\left(x_{0}+x_{3}\right) z_{3}(\bmod 4), \text { if } p=2 .
\end{aligned}
$$

Clearly, $x$ being primitive, $q+r$ is prime to $p$ by choice of $z \bmod p$. Finally, the case $p|m, p| q, p \| d, c_{p}=-1$, cannot hold with $x$ primitive:

Lemma 5. If $x$ is primitive and $m \mid N x$, then if $p$ is a prime dividing $d$ precisely once and satisfying $c_{p}=-1, m$ cannot be divisible by $p^{2}$.

For by (4) with $\alpha_{3}=1$ and (6),

$$
\begin{aligned}
& N x \equiv x_{0}{ }^{2}+p a_{3}\left(a_{2} x_{1}{ }^{2}+a_{1} x_{2}{ }^{2}\right)+a_{1} a_{2} x_{3}{ }^{2} \equiv 0\left(\bmod p^{2}\right), \\
& N x \equiv x_{0}{ }^{2}+x_{0} x_{3}+x_{3}{ }^{2}+2 a_{3}\left(x_{1}{ }^{2}-x_{1} x_{2}+x_{2}{ }^{2}\right) \equiv 0(\bmod 4),
\end{aligned}
$$

whence $x_{0}$ and $x_{3}$, then $x_{1}$ and $x_{2}$, are seen to be divisible by $p$.

In view of Lemma $5, m$ has the following form:

(7) $\quad m=2^{\mu} m_{1} m_{2}$, where $\mu$ is unrestricted $(\geqq 0)$ if $d$ is odd, $\mu$ is 1 or 0 if $d \equiv 2(\bmod 4)$ and $c_{2}=-1, \mu=0$ otherwise; $m_{1}$ is squarefree, and consists only of odd primes $p$ dividing $d$ once and such that $c_{p}=-1 ; m_{2}$ contains no primes dividing $2 d$.

6. Conditions for the existence of a right-divisor of norm $m$. A method of obtaining the right-divisors if any, of a given norm will now be given.

THEOREM 3. Let $m$ be a nonzero integer represented by some form in the genus 
of $F$, and assume $\$ 5(1), x$ primitive, $m \mid N x$. Then, by an algorithm explained below, every factorization

$$
x=u t,
$$

in which $N t=m$, is associated with a representation of the number 1 by a certain quaternary form in the genus of $F$. Hence, unless the genus of $F$ contains a class of forms which do not represent 1 , there exists a right-divisor t of $x$, of norm $m$ and necessarily (by Theorem 2) unique up to a left unit factor.

By (1), $x \bar{t} \equiv 0 \bmod m$; conversely, if $x \bar{t} \equiv 0$ and $N t=m$, then $x \bar{t}=u m$ with $u$ integral, $x \bar{t} t=u t m, x=u t$. We seek the general solution $t$ of the system of four congruences $x \bar{t} \equiv 0 \bmod m$, with the intention of substituting this general solution in the condition $N t=m$.

If $m$ is even we can use the residue

$$
f \equiv j x_{1}^{2}+x_{1} x_{2}+j x_{2}{ }^{2}+\lambda x_{3}^{2}\left(\bmod 2^{3 \mu}\right)
$$

where $j=0$ and $\lambda$ is odd, except that $j=1$ and $\lambda \equiv 2 \bmod 4$ if $d \equiv 2 \bmod 4$ and $c_{2}=-1$. Simultaneously we can assume that

$$
f \equiv a_{1} x_{1}{ }^{2}+a_{2} x_{2}{ }^{2}+a_{3} x_{3}{ }^{2}\left(\bmod m_{1} m_{2}\right),
$$

where $a_{1}, a_{2}$, and $a_{3} / m_{1}$ are integers prime to $m_{1} m_{2}$, and $c_{p}=\left(-a_{1} a_{2} \mid p\right)=-1$ for each prime $p$ in $m_{1}$.

The four coordinates of $x \bar{t}$ in terms of the $j_{\alpha}$ must be divisible by $m$. In particular, on expanding $\left(x_{0}+x_{3} / 2+\sum i_{\alpha} x_{\alpha}\right)\left(t_{0}+t_{3} / 2-\sum i_{\alpha} t_{\alpha}\right)$ with $\left(a_{\alpha \beta}\right)$ as in (2), and $\left(A_{\alpha \beta}\right)$ as given by

$$
\operatorname{adj} f \equiv \lambda\left(j x_{1}{ }^{2}-x_{1} x_{2}+j x_{2}{ }^{2}\right)+(j-1 / 4) x_{3}{ }^{2},
$$

and then using $i_{1}=j_{1}, i_{2}=j_{2}, i_{3}=j_{3}-1 / 2$, we get

$$
\begin{aligned}
x_{0} t_{0}+\lambda\left(j x_{1}-x_{2}\right) t_{1}+\lambda\left(j x_{2}\right) t_{2}+\left(x_{0}+j x_{3}\right) t_{3} & \equiv 0, \\
x_{1} t_{0}+\left(-x_{0}-x_{3}\right) t_{1}+j x_{3} t_{2}+\left(x_{1}-j x_{2}\right) t_{3} & \equiv 0, \\
x_{2} t_{0}+\left(-j x_{3}\right) t_{1}+\left(-x_{0}\right) t_{2}+\quad\left(j x_{1}\right) t_{3} & \equiv 0, \\
x_{3} t_{0}+\quad \lambda x_{2} t_{1}+\left(-\lambda x_{1}\right) t_{2}+\left(-x_{0}\right) t_{3} & \equiv 0, \bmod 2^{\mu} .
\end{aligned}
$$

For the odd modulus $m_{1} m_{2}$ we can use the $i$-coordinates, since $x_{0}{ }^{\prime}$ and $t_{0}{ }^{\prime}$ are integral $\bmod m_{1} m_{2}$, and have

$$
\begin{aligned}
x_{0}{ }^{\prime} t_{0}^{\prime}+a_{2} a_{3} x_{1} t_{1}+a_{3} a_{1} x_{2} t_{2}+a_{1} a_{2} x_{3} t_{3} & \equiv 0, \\
x_{1} t_{0}^{\prime}-x_{0} t_{1}+a_{1} x_{3} t_{2}-a_{1} x_{2} t_{3} & \equiv 0, \\
x_{2} t_{0}^{\prime}-a_{2} x_{3} t_{1}-x_{0} t_{2}+a_{2} x_{1} t_{3} & \equiv 0, \\
x_{3} t_{0}^{\prime}+a_{3} x_{2} t_{1}-a_{3} x_{1} t_{2}-x_{0} t_{3} & \equiv 0, \bmod p^{r},
\end{aligned}
$$

where $p^{r}$ ranges over each of the prime-powers in $m_{1} m_{2}$. 
For success the following process requires that the number of independent congruences in (4) or (5) be two.

First, let $p \mid m_{1}$, whence $p \mid a_{3}$. Since $N x \equiv x_{0}{ }^{2}+a_{2} a_{3} x_{1}{ }^{2}+a_{3} a_{1} x_{2}{ }^{2}+a_{1} a_{2} x_{3}{ }^{2} \equiv 0$, $x_{0}{ }^{\prime} \equiv x_{3} \equiv 0(\bmod p)$. Hence the matrix of (5) has the first and fourth rows

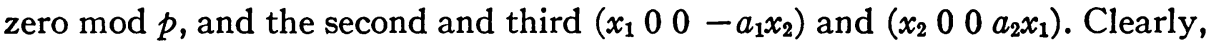
(5) can be solved for $t_{0}{ }^{\prime}$ and $t_{3}\left(\right.$ hence for $t_{0}$ and $\left.t_{3}\right)$ in terms of $t_{1}$ and $t_{2}(\bmod p)$.

Second, let $p=2, d \equiv 2(\bmod 4)$, whence $j=\mu=1$. Then $N x \equiv x_{0}{ }^{2}+x_{0} x_{3}+x_{3}{ }^{2}$ $\equiv 0(\bmod 2), x_{0} \equiv x_{3} \equiv 0(\bmod 2)$, and the matrix of $(4)$ reduces mod 2 like the preceding case; we can solve for $t_{0}$ and $t_{3}$ in terms of $t_{1}$ and $t_{2} \bmod 2$.

If $p \nmid d$, it may be remarked that (4) or (5) will usually involve three independent congruences (if $p^{r} \mid N x$ and $p \nmid x$ ). However, if $p^{r} \mid x_{0}^{\prime}$ or $2^{\mu} \mid 2 x_{0}+x_{3}$, we shall prove that the number of independent congruences is two. For example, if $p \nmid x_{3}$, multiplying $\left(5_{2}\right),\left(5_{3}\right)$, and $\left(5_{4}\right)$ by $a_{2} a_{3} x_{1}, a_{1} a_{3} x_{2}$, and $a_{1} a_{2} x_{3}$, and adding, we get $0 \bmod p^{r}$, whence $\left(5_{4}\right)$ is a consequence of $\left(5_{2}\right)$ and $\left(5_{3}\right)$; using $x_{3},-a_{3} x_{2}$, and $a_{3} x_{1}$ on $\left(5_{1}\right),\left(5_{2}\right)$, and $\left(5_{3}\right)$ shows the same for $\left(5_{1}\right)$. As $p \nmid x_{3}$ and $p \mid a_{2} a_{3} x_{1}{ }^{2}+a_{3} a_{1} x_{2}{ }^{2}+a_{1} a_{2} x_{3}{ }^{2}$ the determinant of the coefficients of $t_{0}{ }^{\prime}$ and $t_{3}$ in $\left(5_{2}\right)$ and $\left(5_{3}\right)$ is prime to $p$, an'd we can solve for $t_{0}{ }^{\prime}$ and $t_{3}$ in terms of $t_{1}$ and $t_{2} \bmod p^{r}$. Similarly in $(4)$ if $x_{3} \equiv-2 x_{0}\left(\bmod 2^{\mu}\right)$, then as $j=0$ and $2^{\mu} \mid-x_{0}^{2}-\lambda x_{1} x_{2},\left(4_{1}\right)$ and $\left(4_{2}\right)$ are proportional, and $\left(\lambda x_{2}\right)\left(4_{2}\right)+\left(\lambda x_{1}\right)\left(4_{3}\right)$ $-x_{0}\left(4_{4}\right) \equiv 0$.

To secure $p^{r} \mid x_{0}^{\prime}$ and $2^{\mu} \mid 2 x_{0}+x_{3}$, we use Lemma $2 \mathrm{~b}$, and the following lemma.

Lemma 6. If $p \nmid d$, and $x$ is primitive, we can find an integral quaternion residue $w \bmod p^{\circ}(s$ given not less than 0$)$, such that $N w$ is prime to $p$, and the real part $2(w x)_{0}$ of $2 w x$ is divisible by $p^{s}$.

We use (2) with $j=0$ and modulus $2^{s}$; (3) with $a_{3}$ prime to $p$ and modulus $p^{*}$. Hence if $p=2, N w \equiv w_{0}^{2}+w_{0} w_{3}-w_{1} w_{2}(\bmod 2)$, and

$$
2(w x)_{0} \equiv w_{0}\left(2 x_{0}+x_{3}\right)+w_{1}\left(\lambda x_{2}\right)+w_{2}\left(\lambda x_{1}\right)+w_{3}\left(x_{0}+x_{1}\right), \bmod 2^{s},
$$

and if $p>2, N w \equiv w_{0}^{\prime 2}+a_{2} a_{3} w_{1}^{2}+a_{3} a_{1} w_{2}^{2}+a_{1} a_{2} w_{3}^{2}(\bmod p)$, and

$$
(w x)_{0} \equiv w_{0}^{\prime} x_{0}^{\prime}-a_{2} a_{3} w_{1} x_{1}-a_{3} a_{1} w_{2} x_{2}-a_{1} a_{2} w_{3} x_{3}\left(\bmod p^{8}\right) .
$$

Since $x$ is primitive, (6) can be solved for one of the $w_{i}$, say $w_{1} \equiv b_{0} w_{0}+b_{2} w_{2}$ $+b_{3} w_{3}\left(\bmod 2^{s}\right)$. This implies mod 2 that $w_{1}=2 v_{1}+b_{0} v_{0}+b_{2} v_{2}+b_{3} v_{3}, w_{0}=v_{0}$, $w_{2}=v_{2}, w_{3}=v_{3}$. Substituting this in the expression for $N w$ gives a quaternary form of determinant $(d / 4)^{2} \cdot 2^{2}$, whence not every term in $v_{0}, v_{2}, v_{3}$ has an even coefficient. Hence $N w$ can be made odd by specifying $v_{0}, v_{2}$, and $v_{3} \bmod 2$, and then $2(w x)_{0} \equiv 0 \bmod 2^{*}$ by choice of $w_{1}$. A similar argument applies to (7).

To sum up, for each prime-power $p^{r}$ in $m$, the condition $x \bar{t} \equiv 0 \bmod p^{r}$ reduces to a pair of congruences such as $t_{0} \equiv \alpha t_{2}+\beta t_{3}, t_{1} \equiv \gamma t_{2}+\delta t_{3}\left(\bmod p^{r}\right)$, where $\alpha, \beta, \gamma, \delta$ are integers; or what is the same thing, to

$$
t_{0}=p^{r} s_{0}+\alpha s_{2}+\beta s_{3}, \quad t_{1}=p^{r} s_{1}+\gamma s_{2}+\delta s_{3}, \quad t_{2}=s_{2}, \quad t_{3}=s_{3},
$$


in which the $s_{i}$ are arbitrary integers, a substitution of determinant $p^{2 r}$. If these last expressions are substituted for the $t_{i}$ in the system of congruences corresponding to $x \bar{t} \equiv 0\left(\bmod p_{2}^{r^{2}}\right)$, where $p_{2}{ }^{r_{2}}$ is another prime-power in $m$, we obtain four congruences in $s_{0}, \cdots, s_{3}$ equivalent, since $p$ and $p_{2}$ are coprime, to $x \bar{t} \equiv 0\left(\bmod p_{2}{ }^{r 2}\right)$, and hence having a solution of determinant $p_{2}{ }^{2 r 2}$ expressing the $s_{i}$ in terms of four new integer parameters. Continuing in this way, and finally compounding the linear substitutions, we see that the general solution of $x \bar{t} \equiv 0(\bmod m)$ is given by a system of the type

$$
t_{i}=\sum_{j=0}^{3} p_{i j} z_{j}, \quad i=0, \cdots, 3, p_{i j} \text { integers, }\left|p_{i j}\right|=m^{2} .
$$

Furthermore, for arbitrary integers $z_{0}, \cdots, z_{3}$ the $t_{i}$ determined by (9) must satisfy

$$
\left(t_{0}+2^{-1} \sum \epsilon_{\alpha} t_{\alpha}\right)^{2}+\sum A_{\alpha \beta} t_{\alpha} t_{\beta} \equiv 0(\bmod m) .
$$

For they satisfy $x \bar{t} \equiv 0, x \bar{t} \equiv 0, x(N t) \equiv 0$, where $x$ is primitive and $N t$ is a rational integer.

On substituting the expressions (9) for $t_{i}$ in the equation

$$
\left(t_{0}+2^{-1} \sum \epsilon_{\alpha} t_{\alpha}\right)^{2}+\sum A_{\alpha \beta} t_{\alpha} t_{\beta}=m,
$$

we obtain an equation of the form

$$
\sum r_{i j} z_{i} z_{j}=m,
$$

where the form on the left has integral coefficients. Also, by the preceding paragraph this form has a value divisible by $m$ for all integers $\boldsymbol{z}_{\boldsymbol{i}}$ and $\boldsymbol{z}_{\boldsymbol{j}}$. It follows that $m$ divides every $r_{i j}$. Setting $r_{i j}=m s_{i j}$ we get

$$
\sum s_{i} z_{i} z_{j}=1 \text {. }
$$

Conversely, for any solution $z_{0}, \cdots, z_{3}$ of $(12)$, the integers $t_{i}$ determined by (9) satisfy $(10)$ along with $x \bar{t} \equiv 0(\bmod m)$, hence $(1)$.

Finally, to prove that $F$ and $G=\sum s_{i j} x_{i} x_{j}$ are in the same genus, we need only show that they have the same index, the same determinant $d^{2} / 16$, and the same form-residues modulo $d^{2}$. Let $F$ and $G$ stand for their own matrices. Since $G$ was obtained from $F$ by applying the transformation (9) of matrix $P=\left(p_{i j}\right)$, and cancelling $m$, we have

$$
P^{\prime} F P=m G .
$$

By (13), since $|P|=m^{2}$, the determinants of $F$ and $G$ are equal. Also, the indices are equal, since $P$ is real, $m$ is positive if $F$ is definite, and the indices of $F$ and $-F$ are the same if $F$ is indefinite.

We assumed that $m$ is representable by the genus of $F$. Hence $m$ $=F\left(v_{0}, v_{1}, v_{2}, v_{3}\right)$, where the $v_{i}$ are rational numbers of denominator prime to $2 d$. Thus $m=N v$, and $m F=N(v t)$ determines a linear transformation of 
determinant $m^{2}$ of $F$ into $m F$, with rational coefficients of denominators prime to $2 d$. By (13), $P^{\prime} m F P=m^{2} G$; hence $F$ can be carried into $G$ by a rational transformation with denominators divisible by $m$ but by no other prime factors of $2 d$. Hence, first, $F$ and $G$ have the same values for all their rational invariants $c_{p}$, and, second, it remains only to prove that $F$ and $G$ are equivalent $\bmod p^{r}$, where $p$ divides both $m$ and $2 d$. That the last holds true follows from the easily proved lemma:

LEMMA 7. Let $p \nmid d ;$ or $p \| d$ and $c_{p}=-1$. Then every integral quaternary form of determinant $d^{2} / 16$ is equivalent to a form with the following residue mod $p^{r}$ :

$$
\begin{gathered}
x_{0} x_{1}+d^{2} x_{2} x_{3}, \quad \text { if } p=2 \text { and } d \text { is odd; } \\
x_{0}^{2}+x_{0} x_{1}+x_{1}^{2}+\left(d^{2} / 18\right)\left(x_{2}^{2}+x_{2} x_{3}+x_{3}^{2}\right), \quad \text { if } p=2 \| d \text { and } c_{2}=-1 \\
x_{0}{ }^{2}+n x_{1}{ }^{2}+p\left(x_{2}^{2}+n x_{3}^{2}\right), \quad \text { if } p>2, p \| d \text { and } c_{p}=-1 .
\end{gathered}
$$

In (16), $-n$ denotes a certain quadratic non-residue mod $p$.

This completes the proof of Theorem 3 . The reader's attention should be drawn at this point to Theorems 11,12 , and 13 .

When $f$ is indefinite, then at least when adj $f$ is fundamental, it follows from a well known theorem of A. Meyer [3(c), p. 54] and $\operatorname{adj} f$ is in a genus of one class. But it does not necessarily follow that this is true of $F$. However, Latimer's theorem that, in the indefinite and fundamental case, every onesided ideal in an integral set is principal implies that if $F$ is indefinite and fundamental, $F$ is in a genus of one class. For, according to Brandt $[2$, p. 29], the ideal-classes correspond to the classes in the genus of $F$.

We shall in $\$ 15$ prove a result showing that Theorem 3 is in some measure best possible.

A word should be added here about the restriction on $m$ in Theorem 3 . The genus of $F$ represents all integers $m$ (having the necessary sign if $F$ is definite) for which $F\left(x_{0}, x_{1}, x_{2}, x_{3}\right) \equiv m(\bmod k)$ is solvable for every modulus $k$. It is easily seen that if $F$ is fundamental, and in certain other cases, this congruence is solvable for every $k$. Hence in this case there is no restriction on $m$, except for sign when $F$ is definite.

7. The automorphs of adj $f$ and $F$. The integral automorphs of any integral ternary quadratic form can be expressed most conveniently by means of our systems of integral quaternions. If $\operatorname{adj} f$ is fundamental the result will be surprisingly precise.

We first make some remarks by way of orientation. The automorphs of $g$ and $\kappa g$ are the same for any form $g$ and constant $\kappa$. The automorphs of $f$ are the transposes of those of adj $f$. For if $A=\operatorname{adj} a$, and $\Delta=|a|, S^{\prime} A S=A$ implies $S^{\prime} A S a=\Delta I, S^{\prime} A$ and $S A$ are permutable, hence $S a S^{\prime}=a$. If there is one automorph of determinant -1 , all such are obtained by multiplying it on one side by each positive automorph, where positive signifies determinant +1 . 
The automorph $-I$ can be so used if the number of variables is odd. The form $F$ has the automorph

$$
\left[\begin{array}{rr}
1 & \epsilon^{\prime} \\
0 & -I
\end{array}\right]
$$

$I$ of order 3 , of determinant -1 . Hence the number of integral automorphs of $F$ is double the number of its integral positive automorphs.

Our main result in this section is the following:

Theorem 4. Let $f$ be integral and adj $f$ be fundamental. Then every positive integral automorph $y_{\alpha}=\sum e_{\alpha \beta} x_{\beta}$ of adj $f$ is obtained by equating $i$-coordinates in

$$
y=(\bar{t} x t) / N t
$$$$
\left(x=\sum i_{\alpha} x_{\alpha}, y=\sum i_{\alpha} y_{\alpha}\right) \text {, }
$$

as $t$ ranges over the primitive quaternions such that $N t \mid d$. Every positive integral automorph $y_{i}=\sum h_{i j} x_{j}$ of $F=\left(x_{0}+2^{-1} \sum \epsilon_{\alpha} x_{\alpha}\right)^{2}+\sum A_{\alpha \beta} x_{\alpha} x_{\beta}$ is obtained by equating $j$-coordinates in

$$
y=(t x u) / N t \quad\left(x=x_{0}+\sum j_{\alpha} x_{\alpha}, y=y_{0}+\sum j_{\alpha} y_{\alpha}\right),
$$

as $t$ and $u$ range over the primitive quaternions such that $N t=N u$ and $N t \mid d$. All automorphs so obtained are integral, and each appears exactly twice, once for $t$ and once for $-t$ in (2), once for $(t, u)$ and once for $(-t,-u)$ in (3).

TheOREM 5. If $f$ is integral and adj $f$ is not fundamental, then all the positive integral automorphs of adj $f$, or $F$, are included among the automorphs obtained as in Theorem 4; but among those so obtained there may be some which are not integral.

We consider first the uniqueness property. If we apply to adj $f$ a nonsingular transformation $T$, as in Theorem 1, all quaternion equations will be transformed uniquely, and the automorphs will correspond as $E$ to $T^{-1} E T$. Hence we can suppose if we wish that

$$
f=a_{1} x_{1}{ }^{2}+a_{2} x_{2}{ }^{2}+a_{3} x_{3}{ }^{2}, \quad a_{1} a_{2} a_{3} \neq 0 .
$$

The expression (2) is homogeneous: it is unchanged if $t$ is replaced by $\lambda t$, $\lambda$ scalar. Conversely, we prove the following lemma.

LEMma 8. If the coefficient field has characteristic not 2 , then $\bar{t} x t / N t=\bar{u} x u / N u$ identically in $x$ implies that $t$ and $u$ differ only by a scalar factor, which may however lie in a larger field.

We can choose $\lambda$ to make $N t=\lambda^{2} N u$, and so can assume $N t=N u$. We shall verify that if the values of the nine elements of $\bar{t} x t$ are fixed, as well as the value of $N t$, then $t_{i} t_{j}$ are uniquely determined $(i, j=0,1,2,3)$, whence $\pm t$ is determined. The explicit expansion of (2) is $D / N t$, where $D$ is

(5) $\left[\begin{array}{ccc}t_{0}^{2}+a_{2} a_{3} t_{1}^{2}-a_{3} a_{1} t_{2}{ }^{2}-a_{1} a_{2} t_{3}^{2} & 2 a_{1}\left(t_{0} t_{3}+a_{3} t_{1} t_{2}\right) & 2 a_{1}\left(-t_{0} t_{2}+a_{2} t_{3} t_{1}\right) \\ 2 a_{2}\left(-t_{0} t_{3}+a_{3} t_{1} t_{2}\right) & t_{0}^{2}-a_{2} a_{3} t_{1}^{2}+a_{3} a_{1} t_{2}{ }^{2}-a_{1} a_{2} t_{3}{ }^{2} & 2 a_{2}\left(t_{0} t_{1}+a_{1} t_{2} t_{3}\right) \\ 2 a_{3}\left(t_{0} t_{2}+a_{2} t_{1} t_{3}\right) & 2 a_{3}\left(-t_{0} t_{1}+a_{1} t_{2} t_{3}\right) & t_{0}^{2}-a_{2} a_{3} t_{1}^{2}-a_{3} a_{1} t_{2}^{2}+a_{1} a_{2} t_{3}^{2}\end{array}\right]$. 
Clearly the value of $t_{0}{ }^{2}+a_{2} a_{3} t_{1}{ }^{2}+a_{3} a_{1} t_{2}{ }^{2}+a_{1} a_{2} t_{3}{ }^{2}(=N t)$ and the values of the three diagonal elements fix the values $4 t_{i}{ }^{2}$, and the nondiagonal elements determine $4 t_{i} t_{j}$.

Abbreviate $A_{\alpha \alpha}$ as $A_{\alpha}$. Equating $i$-coordinates in $y=t x u$ we get $y_{i}=\sum r_{i j} x_{j}$, where

$$
\begin{array}{ll}
r_{00}=t_{0} u_{0}-A_{1} t_{1} u_{1}-A_{2} t_{2} u_{2}-A_{3} t_{3} u_{3}, & r_{01}=-A_{1}\left(t_{0} u_{1}+t_{1} u_{0}-a_{1} t_{2} u_{3}+a_{1} t_{3} u_{2}\right), \\
r_{02}=-A_{2}\left(t_{0} u_{2}+t_{2} u_{0}+a_{2} t_{1} u_{3}-a_{2} t_{3} u_{1}\right), & r_{03}=-A_{3}\left(t_{0} u_{3}+t_{3} u_{0}-a_{3} t_{1} u_{2}+a_{3} t_{2} u_{1}\right), \\
r_{10}=t_{0} u_{1}+t_{1} u_{0}+a_{1} t_{2} u_{3}-a_{1} t_{3} u_{2}, & r_{11}=t_{0} u_{0}-A_{1} t_{1} u_{1}+A_{2} t_{2} u_{2}+A_{3} t_{3} u_{3}, \\
r_{12}=a_{1}\left(t_{0} u_{3}-t_{3} u_{0}-a_{3} t_{1} u_{2}-a_{3} t_{2} u_{1}\right), & r_{13}=-a_{1}\left(t_{0} u_{2}-t_{2} u_{0}+a_{2} t_{1} u_{3}+a_{2} t_{3} u_{1}\right), \\
r_{20}=t_{0} u_{2}+t_{2} u_{0}-a_{2} t_{1} u_{3}+a_{2} t_{3} u_{1}, & r_{21}=-a_{2}\left(t_{0} u_{3}-t_{3} u_{0}+a_{3} t_{1} u_{2}+a_{3} t_{2} u_{1}\right), \\
r_{22}=t_{0} u_{0}+A_{1} t_{1} u_{1}-A_{2} t_{2} u_{2}+A_{3} t_{3} u_{3}, & r_{23}=a_{2}\left(t_{0} u_{1}-t_{1} u_{0}-a_{1} t_{2} u_{3}-a_{1} t_{3} u_{2}\right), \\
r_{30}=t_{0} u_{3}+t_{3} u_{0}+a_{3} t_{1} u_{2}-a_{3} t_{2} u_{1}, & r_{31}=a_{3}\left(t_{0} u_{2}-t_{2} u_{0}-a_{2} t_{1} u_{3}-a_{2} t_{3} u_{1}\right), \\
r_{32}=-a_{3}\left(t_{0} u_{1}-t_{1} u_{0}+a_{1} t_{2} u_{3}+a_{1} t_{3} u_{2}\right), & r_{33}=t_{0} u_{0}+A_{1} t_{1} u_{1}+A_{2} t_{2} u_{2}-A_{3} t_{3} u_{3} .
\end{array}
$$

The terms occur in sets of four; for example, the $r_{i i}$ involve $t_{0} u_{0}$ and $A_{\alpha} t_{\alpha} u_{\alpha}$. The signs in different $r_{i j}$ of a set differ in only two terms, so that if the algebraic sum is taken to make a particular term add four times, the other three terms will cancel. Thus if the values $r_{i j}$ are fixed, the values $4 a_{1} a_{2} a_{3} t_{i} u_{j}$ are fixed for every $i$ and $j$. If $N t$ and $N u$ also have fixed nonzero values, either $(t, u)$ or $(-t,-u)$ are thus uniquely determined.

It will be noted that (6) reduces to (5) when $\bar{u}=t$, the first row and column becoming $N t, 0,0,0$.

We saw in $\$ 2$ that the positive rational automorphs of adj $f$ are given by (2), but did not determine the field restrictions on the $t_{i}$. They can indeed be restricted to be rational. For, $f$ can be carried by a rational transformation into a form of type (4) with rational nonzero $a_{\alpha}$. We can choose $\lambda$ so that $N t$ is rational. The discussion following (5) shows that $t_{i} t_{j}$ are rational $(i, j=0,1,2,3)$. Hence $t_{i}=u_{i} s^{1 / 2}$, where the $u_{i}$ are rational, and we can replace $t$ by $u$.

Before going further we prove a similar result for the rational automorphs of $F$.

LEMMA 9. Let $\left(a_{\alpha \beta}\right)$ be rational. Then as $t$ and $u$ range over the rational quaternions associated with $\left(a_{\alpha \beta}\right)$, such that $N u=1 / N t$, then $y=t x u$ ranges over all the positive rational automorphs of $F_{0}=x_{0}{ }^{2}+\sum A_{\alpha \beta} x_{\alpha} x_{\beta}$.

Our proof is similar to that of Hurwitz $[7$, p. 63] for Hamiltonian quaternions. First notice that if the lemma holds for $A=\left(A_{\alpha \beta}\right)$, it holds for $B=T^{\prime} A T$, where $T$ is rational and of positive determinant. For then

$$
S=\left[\begin{array}{ll}
1 & 0 \\
0 & T
\end{array}\right], \text { where } S^{-1}=\left[\begin{array}{ll}
1 & 0 \\
0 & T^{-1}
\end{array}\right],
$$


gives a rational transformation of $F_{0}$ into the new norm-form $G_{0}=y_{0}{ }^{2}$ $+\sum B_{\alpha \beta} y_{\alpha} y_{\beta}$, and all rational automorphs of $G_{0}$ are given by $S^{-1} H S$, where $H$ ranges over all rational automorphs of $F_{0}$. Let $y=t x u$ be the equation which, on equating $A$-coordinates, expands into the matrix equation $y=H x$, where $y^{\prime}=\left(y_{0}, y_{1}, y_{2}, y_{3}\right)$, and so on. Applying the change of basis $T$, consider the corresponding equation $y=t x u$ in $B$-quaternions. The $A$-coordinates of $y$ and $x$ are respectively $y_{0}$ and $T \eta, x_{0}$ and $T \xi$, that is, $S y$ and $S x$. Hence the result of equating $B$-coordinates in $y=t x u$ must be the system of linear equations $S y=H S x$, or $y=S^{-1} H S x$, which is any desired automorph of $G_{0}$.

If $y_{i}=\sum h_{i j} x_{j}$ is a rational automorph of $F_{0}$, then multiplying by $1, i_{1}, i_{2}, i_{3}$ and adding, we get $y=h_{0} x_{0}+h_{1} x_{1}+h_{2} x_{2}+h_{3} x_{3}$, where $h_{j}=h_{0 j}+h_{1 j} i_{1}+h_{2 j} i_{2}+h_{3 j} i_{3}$ are rational, linearly independent quaternions; and we see that the result of substituting for $y$ in $y \bar{y}$ or $y_{0}{ }^{2}+\sum A_{\alpha \beta} y_{\alpha} y_{\beta}$ must be $x_{0}{ }^{2}+\sum A_{\alpha \beta} x_{\alpha} x_{\beta}$. The problem of finding rational automorphs reduces to that of finding rational quaternions $h_{j}$ satisfying

$$
\left(h_{0} x_{0}+h_{1} x_{1}+h_{2} x_{2}+h_{3} x_{3}\right)\left(\bar{h}_{0} x_{0}+\bar{h}_{1} x_{1}+\bar{h}_{2} x_{2}+\bar{h}_{3} x_{3}\right)=x_{0}^{2}+\sum A_{\alpha \beta} x_{\alpha} x_{\beta},
$$

identically in $x_{0}, x_{1}, x_{2}, x_{3}$. Comparing coefficients of $x_{0}{ }^{2}$ we have $h_{0} \bar{h}_{0}=1$, and we can define rational quaternions $k_{\alpha}$ by the equations

$$
h_{1}=k_{1} h_{0}, \quad h_{2}=k_{2} h_{0}, \quad h_{3}=k_{3} h_{0} .
$$

Hence (8) reduces to

$$
\left(x_{0}+k_{1} x_{1}+k_{2} x_{2}+k_{3} x_{3}\right)\left(x_{0}+\bar{k}_{1} x_{1}+\bar{k}_{2} x_{2}+\bar{k}_{3} x_{3}\right)=x_{0}^{2}+\sum A_{\alpha \beta} x_{\alpha} x_{\beta} .
$$

On equating coefficients we see that

$$
k_{\alpha}+\bar{k}_{\alpha}=0, \quad k_{\alpha} \bar{k}_{\alpha}=A_{\alpha \alpha}, \quad k_{\alpha} \bar{k}_{\beta}+k_{\beta} \bar{k}_{\alpha}=2 A_{\alpha \beta} \quad(\alpha, \beta=1,2,3) .
$$

We now assume $A_{\alpha \beta}=0$ if $\alpha \neq \beta$, although this may not be strictly necessary. Then on eliminating the $\bar{k}_{\alpha}$, we get

$$
k_{\alpha}^{2}=-A_{\alpha \alpha}, \quad k_{2} k_{3}=-k_{3} k_{2}, \quad k_{3} k_{1}=-k_{1} k_{3}, \quad k_{1} k_{2}=-k_{2} k_{1} .
$$

Hence

$$
\overline{k_{1} k_{2} k_{3}}=\bar{k}_{3} \bar{k}_{2} \bar{k}_{1}=-k_{3} k_{2} k_{1}=k_{2} k_{3} k_{1}=-k_{2} k_{1} k_{3}=k_{1} k_{2} k_{3} .
$$

Hence the quaternion $k_{1} k_{2} k_{3}$ is equal to its conjugate, and must be real. Since $N\left(k_{1} k_{2} k_{3}\right)=A_{11} A_{22} A_{33}=\left(a_{11} a_{22} a_{33}\right)^{2}$, we can set

$$
k_{1} k_{2} k_{3}=\sigma a_{11} a_{22} a_{33},
$$

$$
\sigma= \pm 1
$$

From (13) follows $k_{2} k_{3}=-\sigma a_{11} k_{1}, k_{3} k_{1}=-\sigma a_{22} k_{2}, k_{1} k_{2}=-\sigma a_{33} k_{3}$. Hence the three quaternions $\sigma k_{1}, \sigma k_{2}, \sigma k_{3}$ satisfy the same multiplication table as the $i_{\alpha}$. Hence we can choose $q$ and $\sigma$ to satisfy (10) of $\$ 2$. Accordingly, in (8),

$$
y=q x q^{-1} h_{0}, \quad \text { or } \quad y=q \bar{x} q^{-1} h_{0},
$$


that is, $y=t x u$ or $y=t \bar{x} u$, where $t$ and $u$ are rational quaternions such that $N t \cdot N u=1$. Continuity considerations show that the determinant is +1 for the first, -1 for the second of these transformations. Lemma 9 follows.

If $t$ has rational coordinates we can choose a proportionality factor $\lambda$ to make $t$ integral and primitive. Hence every positive rational automorph of $\operatorname{adj} f$ is given by $y=\bar{t} x t / N t$ with $t$ primitive. We must see whether the various prime-powers in $N t$ can be cancelled to make the coefficients $\bar{t} i_{1} t / N t$, $\bar{t} i_{2} t / N t, \bar{t} i_{3} t / N t$ of the $x_{\alpha}$ have integer coordinates. Similarly, every positive rational automorph of $F$ is obtained by equating $j$-coordinates in

$$
y=t x u / m \text {, }
$$

where $t$ and $u$ are primitive quaternions, $m$ a nonzero integer, $N t \cdot N u=m^{2}$.

LeMma 10. Let $t, u$ denote primitive quaternions, $N(t u)=m^{2}$. If $t x u / m$ is integral for every integral quaternion $x$, then: (a) $N t=N u= \pm m$; (b) $u=\bar{t} \theta$, where $\theta$ is a unit; (c) $t x \bar{t} / m$ is integral for every integral $x$.

For, in particular, $t u \equiv 0(\bmod m)$. If $p^{s}$ is a prime-power in $m$, then $N t \cdot N u$ is divisible precisely by $p^{2 s}$. We prove that $N t$ and $N u$ are each divisible by $p^{s}$. For if not, let $N t$ be divisible only by $p^{n}, n<s$. Then $\bar{t} t u \equiv 0\left(\bmod p^{s}\right)$, $u \equiv 0\left(\bmod p^{s-n}\right)$, contradicting the primitivity of $u$. Hence $N t=N u= \pm m$. Again, $t u= \pm m \theta, \theta$ integral. Since $N(t u)=m^{2} N \theta, \theta$ is a unit. Thus $u=\tilde{t} \theta$. The integrality of $t x \bar{t} \theta / m$ implies that of $t x \bar{t} \theta \bar{\theta} / m$, that is, of $t x \bar{t} / m$, for every integral $x$.

LEMma 11. Let $t, u$ be primitive, $N t=N u= \pm m, m \mid d$, adj $f$ be fundamental. Then $t x u / m$ is integral for every integral quaternion $x$. Also, $t x \bar{t} / m$ is purelyintegral for every purely-integral $x$.

Let $p>2$. We can assume that (4) holds as a congruence mod $p^{r}, r$ large, and can use (5) and (6) to see whether the power of $p$ in $N t$ cancels. Since adj $f$ is fundamental, $p$ divides $d$ precisely once and $c_{p}=-1$; we can suppose $p \| a_{3}$ and $\left(-a_{1} a_{2} \mid p\right)=-1$. Then if $p \mid N t$ (necessarily only once) clearly $u_{0} \equiv u_{3} \equiv t_{0} \equiv t_{3} \equiv 0(\bmod p)$, and every element of (5) and (6) is obviously divisible by $p$.

Note in advance for Lemma 13, that if $p^{8}$ exceeds the power of $p$ in $d$ ( $\equiv 4 a_{1} a_{2} a_{3}$ ), then by arguments like those following (5) and (6), $p^{s}$ cannot divide every element of (5), or (6), without rendering $t$, or $t$ or $u$, imprimitive. This applies equally to the prime 2 in case (4) holds, mod $2^{r}$.

To facilitate the discussion of the prime 2 we need explicit expansions of (2) and (3), especially in the case $f=j x_{1}{ }^{2}+x_{1} x_{2}+j x_{2}{ }^{2}+\lambda x_{3}{ }^{2}, j=0$ or 1 . These can be used with the elements determined mod $2^{r}$, when the expression for $f$ is only a residue mod $2^{r}$. To derive these expansions we take $a_{1}=4 j-1, a_{2}=1$, $a_{3}=\lambda$, and so derive the adjoint form

$$
\lambda\left(j x_{1}{ }^{2}-x_{1} x_{2}+j x_{2}{ }^{2}\right)+(j-1 / 4) x_{3}{ }^{2}
$$


from $a_{2} a_{3} x_{1}^{2}+a_{3} a_{1} x_{2}^{2}+a_{1} a_{2} x_{3}^{2}$ by the substitution

$$
T=\frac{1}{2}\left[\begin{array}{ccc}
1+j & -1 & 0 \\
1-j & 1 & 0 \\
0 & 0 & 1
\end{array}\right], \quad T^{-1}=\left[\begin{array}{ccc}
1 & 1 & 0 \\
j-1 & 1+j & 0 \\
0 & 0 & 2
\end{array}\right],
$$

of determinant $1 / 4$. Then we form $T^{-1} E T$, using $E$ as in (5), and apply $T$ to $t$, that is, put $t_{0}=u_{0}, t_{1}=(1+j) u_{1} / 2-u_{2} / 2, t_{2}=(1-j) u_{1} / 2+u_{2} / 2, t_{3}=u_{3} / 2$. Finally, we replace $u_{0}$ by $u_{0}+u_{3} / 2$, so that the $u_{i}$ will be $j$-coordinates and integrality most easily discussed. If $j=0$, the result for the positive automorphs of $\operatorname{adj} f$ is

$$
\frac{1}{N u}\left[\begin{array}{ccc}
u_{0}^{2} & -\lambda u_{1}^{2} & u_{0} u_{1} \\
-\lambda u_{2}^{2} & \left(u_{0}+u_{3}\right)^{2} & -u_{2}\left(u_{0}+u_{3}\right) \\
2 \lambda u_{0} u_{2} & -2 \lambda u_{1}\left(u_{0}+u_{3}\right) & u_{0}^{2}+u_{0} u_{3}+\lambda u_{1} u_{2}
\end{array}\right],
$$

where $N u=u_{0}^{2}+u_{0} u_{3}-\lambda u_{1} u_{2}$ (the Brandt-norm); and if $j=1$ the result is the quotient by $N u\left(=u_{0}^{2}+u_{0} u_{3}+u_{3}^{2}+\lambda\left(u_{1}^{2}-u_{1} u_{2}+u_{2}^{2}\right)\right)$ of

$$
\left[\begin{array}{ccc}
u_{0}^{2}-u_{3}^{2}+\lambda\left(u_{1}^{2}-u_{2}^{2}\right) & 2 u_{0} u_{3}+u_{3}^{2}+\lambda\left(2 u_{1} u_{2}-u_{1}^{2}\right) & u_{0} u_{1}+2 u_{1} u_{3}-2 u_{0} u_{2}-u_{2} u_{3} \\
-2 u_{0} u_{3}-u_{3}^{2}+\lambda\left(2 u_{1} u_{2}-u_{2}^{2}\right) & 2 u_{0} u_{3}+u_{0}^{2}-\lambda\left(u_{1}^{2}-u_{2}^{2}\right) & 2 u_{0} u_{1}+u_{1} u_{3}-u_{0} u_{2}+u_{2} u_{8} \\
2 \lambda\left(u_{0} u_{2}+u_{1} u_{3}\right) & -2 \lambda\left(u_{0} u_{1}+u_{1} u_{3}-u_{2} u_{3}\right) & u_{0}^{2}+u_{0} u_{3}+u_{3}^{2}-\lambda\left(u_{1}^{2}-u_{1} u_{2}+u_{2}^{2}\right)
\end{array}\right] \text {. }
$$

Now (6) is obtained from $y=t x u$, for the $f$ in (4), by equating $i$-coordinates. To get the result of equating $j$-coordinates in $y_{0}+\sum j_{\alpha} y_{\alpha}=\left(t_{0}+\sum j_{\alpha} t_{\alpha}\right)$ - $\left(x_{0}+\sum j_{\alpha} x_{\alpha}\right)\left(u_{0}+\sum j_{\alpha} u_{\alpha}\right)$, we write it as $y_{0}+y_{3} / 2+\sum i_{\alpha} y_{\alpha}=\left(t_{0}+t_{3} / 2+\sum i_{\alpha} t_{\alpha}\right)$ - $\left(x_{0}+x_{3} / 2+\sum i_{\alpha} x_{\alpha}\right)\left(u_{0}+u_{3} / 2+\sum i_{\alpha} u_{\alpha}\right)$, and apply the transformation $T$ as above, using (6).

Using the abbreviations

$$
p_{1}=t_{2} u_{3}+t_{3} u_{2}, \quad q_{1}=t_{2} u_{3}-t_{3} u_{2}, \quad r_{1}=t_{0} u_{1}+t_{1} u_{0}, \quad s_{1}=t_{0} u_{1}-t_{1} u_{0} \text {. }
$$

where subscripts $1,2,3$ are to be permuted cyclically, and also $\rho=t_{1} u_{1}+t_{2} u_{2}$. and $\sigma=t_{1} u_{1}-t_{2} u_{2}$, we thus find for the automorphs of $F=x_{0}{ }^{2}+x_{0} x_{3}+j x_{3}{ }^{2}$ $+\lambda\left(j x_{1}{ }^{2}-x_{1} x_{2}+j x_{2}{ }^{2}\right)=N x$ the expression $\left(h_{i j} / N t\right)$, where

$$
\begin{array}{lll}
h_{00}=t_{0} u_{0}+\lambda t_{2} u_{1}-j\left(t_{3} u_{3}+\lambda \rho\right), & h_{01}=\lambda\left(t_{2} u_{0}-j r_{1}+j q_{1}\right), \\
h_{02}=\lambda\left(t_{0} u_{1}-j r_{2}+j q_{2}\right), & & h_{03}=\lambda t_{2} u_{1}-j\left(r_{3}+t_{3} u_{3}+\lambda \rho-\lambda q_{3}\right) ; \\
h_{10}=r_{1}+t_{3} u_{1}+j q_{1}, & & h_{11}=t_{0} u_{0}+t_{3} u_{0}+j\left(t_{3} u_{3}-\lambda \sigma\right), \\
h_{12}=\lambda t_{1} u_{1}+j\left(s_{3}-\lambda p_{3}\right), & & h_{13}=t_{0} u_{1}+t_{3} u_{1}-j\left(s_{2}+p_{2}-q_{1}\right) ; \\
(20) & h_{20}=r_{2}+t_{2} u_{3}+j q_{2}, & h_{21}=\lambda t_{2} u_{2}-j\left(s_{3}+\lambda p_{3}\right), \\
h_{22}=t_{0} u_{0}+t_{0} u_{3}+j\left(t_{3} u_{3}+\lambda \sigma\right), & h_{23}=t_{2} u_{0}+t_{2} u_{3}+j\left(s_{1}+q_{2}-p_{1}\right) ; \\
h_{30}=r_{3}+t_{3} u_{3}+\lambda q_{3}, & h_{31}=\lambda\left(s_{2}+t_{3} u_{2}-j p_{2}\right), \\
h_{32}=-\lambda\left(s_{1}-t_{1} u_{3}+j p_{1}\right), & \\
h_{33}=t_{0} u_{0}+r_{3}+t_{3} u_{3}-\lambda t_{2} u_{1}+j\left(\lambda \rho-t_{3} u_{3}\right) .
\end{array}
$$


These have been checked carefully. As a final check note that (20) can be reduced to (17) and (18) by setting $t=\bar{u}$, that is $t_{0}+t_{3} / 2=u_{0}+u_{3} / 2, t_{1}=-u_{1}$, $t_{2}=-u_{2}, t_{3}=-u_{3}$, hence $t_{0}=u_{0}+u_{3}$; the first element in the last three rows of $\left(h_{i j}\right)$ then becomes zero, and the other elements become those of (17), (18); also, adding half the last row to the first gives $y_{0}+y_{3} / 2=N u\left(x_{0}+x_{3} / 2\right)$.

In (18), and in (20) with $j=1,2 \mid N u$ implies that $u_{0}$ and $u_{3}$ are even, and since $\lambda$ is even when $j=1$, it is seen that all elements of (18) and (20) are even, so that $t x \bar{t} / 2$ is purely integral, and $t x u / 2$ is integral. This completes the proof of Lemma 11.

In anticipation of Lemma 13, note that if $2^{s}$ exceeds the power of 2 in $d$ $(=(4 j-1) \lambda)$, then in $(17), 2^{s}$ cannot divide the four leading elements without rendering every $u_{i}$ divisible by 2 ; and in (18), $2^{*}$ cannot divide both $N u$ and the last element, without 2 dividing both $u_{0}{ }^{2}+u_{0} u_{3}+u_{3}{ }^{2}$ and $u_{1}{ }^{2}-u_{1} u_{2}+u_{2}{ }^{2}$, whence $2 \mid u$.

Again, consider (20) with $j$ equal to zero. For any integer $\lambda$, it is easily verified that if $2^{s}$ exceeds the power of 2 in $\lambda$, and $2^{s}$ divides all 16 elements of (20), then every $t_{i} u_{j}$ is even, so that $t$ or $u$ is imprimitive. A good scheme is to construct a 4 -by-4 square, and check off each product $t_{i} u_{j}$ as even, as it appears.

The proof of Theorems 4 and 5 is now complete, at least in the fundamental case, when we observe (Lemma 5) that if $j=1,4$ cannot divide $N t$ if $t$ is primitive.

We now need more complete information on the possible form-residues:

LEMMa 12. Any integral ternary form $f$ can be carried by a unimodular transformation into a form with the following residue mod $2^{r}, r$ large: either (i) as in (4) with integers $a_{\alpha}$, or (ii) as $2^{\delta}\left(j x_{1}{ }^{2}+x_{1} x_{2}+j x_{2}{ }^{2}+\lambda x_{3}{ }^{2}\right)$, or (iii) as $2^{\beta+2}\left(j x_{1}{ }^{2}+x_{1} x_{2}+j x_{2}{ }^{2}\right)+a_{3} x_{3}{ }^{2}$. Here $j=0$ or $1 ; \beta$ and $\delta$ denole non-negative integers, $\lambda$ and $a_{3}$ integers. In (ii) if $j=1, \lambda$ is even. In (iii), the power of 2 in $a_{3}$ does not exceed $2^{\beta}$.

Forming determinants we see that the connection with $d$ is as follows: (i) $d \equiv 4 a_{1} a_{2} a_{3}\left(\bmod 2^{r}\right)$; (ii) $d \equiv(4 j-1) 2^{2 \delta} \lambda$; (iii) $d \equiv(4 j-1) 2^{2 \beta+4} a_{3}\left(\bmod 2^{r}\right)$.

In view of Lemma 10, we can complete the proof of Theorem 5, by proving the following lemma, thus avoiding further complicated fourth order matrices:

LemMa 13. Let $t$ be a primitive quaternion of norm $m$. If either (a) $\bar{t} x t / m$ is purely-integral for every purely-integral $x$, or if (b) $\bar{t} x t / m$ is integral for every integral pure $x$, then $m \mid d$.

The significance of (a) and (b) may be seen as follows. Set $y=\bar{t} x t$, where $x$ and $y$ are pure. Equating coordinates, we have, say,

$$
y_{\alpha}=t_{\alpha 1} x_{1}+t_{\alpha 2} x_{2}+t_{\alpha 3} x_{3} \quad(\alpha=1,2,3),
$$

where $t_{\alpha 1}, t_{\alpha 2}, t_{\alpha 3}$ are the elements of the $\alpha$ th row of the matrices in, for ex- 
ample, (5), (17), or (18). The column $t_{1 \beta}, t_{2 \beta}, t_{3 \beta}$ gives the coordinates of $\bar{t}_{\beta} t$. Now, (a) means that the $y_{\alpha}$ are integers divisible by $m$ for all choices of integers $x_{\alpha}$; that is, $m \mid t_{\alpha \beta}(\alpha, \beta=1,2,3)$. But, in case adj $f$ is given by (16), whence $\epsilon_{1}=\epsilon_{2}=0, \epsilon_{3}=1$, then (b) means that for any integers $x_{1}, x_{2}$, and even integer $x_{3}, y_{1} / m, y_{2} / m$ are integers and $y_{3} / m$ an even integer; that is, $m \mid t_{11}, t_{12}$, $t_{21}, t_{22}, 2 t_{13}, 2 t_{23}, t_{31} / 2, t_{32} / 2$, and $t_{33}$.

Only the power of 2 in $m$, in the nonfundamental cases, remains to be considered. Consider first (ii) with $j=0$ and $\delta$ positive. The form adj $f=2^{2 \delta}\left(-\lambda x_{1} x_{2}-x_{3}{ }^{2} / 4\right)$ is derived from $-\lambda x_{1} x_{2}-x_{3}{ }^{2} / 4$ by the substitution $x_{\alpha}=2^{8} y_{\alpha}$; note that in $T^{-1} E T, T$ is $2^{\delta} I, T^{-1}$ is $2^{-\delta} I$, and $T^{-1} E T=E$. Referring to (17), and replacing $u_{0}$ by $u_{0}-u_{3} / 2$ (cf. last step in getting (17)) before replacing $u_{\alpha}$ by $2^{\delta} u_{\alpha}$, we see that the automorph is now $E_{1} / N u$, where the first four elements of $E_{1}$ are $\left(u_{0}-2^{\delta-1} u_{3}\right)^{2},-\lambda 2^{2 \delta} u_{1}{ }^{2},-\lambda 2^{2 \delta} u_{2}{ }^{2},\left(u_{0}+2^{\delta-1} u_{3}\right)^{2}$; and $N u$ is $u_{0}^{2}-2^{2 \delta-2} u_{3}^{2}-2^{2 \delta} \lambda u_{1} u_{2}$. Clearly, if $2^{8}$ divides these five numbers, but not $d$; every $u_{i}$ is even.

Similarly in (ii) with $j$ equal to 1 , if $\delta>0,2^{s}$ cannot divide $N u=u_{0}^{2}$ $+3 \cdot 2^{2 \delta-2} u_{3}{ }^{2}+2^{2 \delta} \lambda\left(u_{1}{ }^{2}-u_{1} u_{2}+u_{2}{ }^{2}\right)$ and the last diagonal element $u_{0}{ }^{2}+3 \cdot 2^{2 \delta-2} u_{3}{ }^{2}$ $-2^{2 \delta} \lambda\left(u_{1}^{2}-u_{1} u_{2}+u_{2}^{2}\right)$, without rendering $u$ imprimitive.

Finally, in (iii), $\operatorname{adj} f=2^{2 \beta+4}(j-1 / 4) x_{3}{ }^{2}+2^{\beta+2} a_{3}\left(j x_{1}{ }^{2}-x_{1} x_{2}+j x_{2}{ }^{2}\right)$, and is derived from (16) by taking $\lambda=2^{\beta+2} a_{3}$ and replacing $x_{3}$ by $2^{\beta+2} x_{3}$. The third row of (17) or (18) is therefore to be multiplied by $2^{-\beta-2}$, and then the third column by $2^{\beta+2}, u_{0}-u_{3} / 2$ is to be substituted for $u_{0}$, and then $u_{3}$ is to be replaced by $2^{\beta+2} u_{3}$. The new $N u$ is $u_{0}^{2}+(4 j-1) 2^{2 \beta+2} u_{3}^{2}+2^{\beta+2} a_{3}\left(j u_{1}^{2}-u_{1} u_{2}+j u_{2}^{2}\right)$. Now $2^{s}$ exceeds the power of 2 in $2^{2 \beta+4} a_{3}$. Hence if $j=0,2^{s}$ cannot divide the first four elements $\left(u_{0}-2^{\beta+1} u_{3}\right)^{2},-2^{\beta+2} a_{3} u_{1}{ }^{2},-2^{\beta+2} a_{3} u_{1}{ }^{2}$, and $\left(u_{0}+2^{\beta+1} u_{3}\right)^{2}$, without 2 dividing $u$. Finally, if $j=1$, and $2^{s}$ divides $N u$ and the last diagonal element $u_{0}{ }^{2}+3 \cdot 2^{2 \beta+2} u_{3}{ }^{2}-2^{\beta+2} a_{3}\left(u_{1}{ }^{2}-u_{1} u_{2}+u_{2}{ }^{2}\right)$, then we see that $2^{\beta+2} \mid u_{1}{ }^{2}-u_{1} u_{2}+u_{2}{ }^{2}$ and that $u_{0}=2^{\beta+1} v_{0}, 2^{s-3-2 \beta} \mid v_{0}{ }^{2}+3 u_{3}{ }^{2}$. If $a_{3}$ is even, $\mathrm{s} \geqq 2 \beta+6$; and if $a_{3}$ and $\beta$ are odd, $2^{2 \beta+5} \mid 2^{\beta+2} a_{3}\left(u_{1}{ }^{2}-u_{1} u_{2}+u_{2}{ }^{2}\right)$; in these cases $8 \mid v_{0}{ }^{2}+3 u_{3}{ }^{2}$, whence $u_{3}$ is even. But if $a_{3}$ is odd and $\beta$ is even, and $s=2 \beta+5$, then putting $u_{1}=2^{\beta / 2+1} v_{1}, u_{2}=2^{\beta / 2+1} v_{2}, u_{0}=2^{\beta+1} v_{0}$, we find for the first two diagonal elements the expressions $2^{2 \beta+2}\left[\left(v_{0} \mp u_{3}\right)^{2}-4 u_{3}{ }^{2} \pm 4 a_{3}\left(v_{1}{ }^{2}-v_{2}{ }^{2}\right)\right]$. The bracketed expression in both cases must be divisible by 8 . If $u_{3}$ could be odd, then $v_{0}-u_{3}$ and $v_{0}+u_{3}$ would both be congruent to 2 , or both to $0, \bmod 4$. Neither case is possible, and $u_{3}$ must be even. This completes the proof of Theorem 5.

TheOREM 6. Let adj $f$ be fundamental, that is, let $d$ be squarefree and $c_{p}$ be -1 for each prime $p$ in $d$. Then: (i) if $m \mid d$, all solutions $t$ of $N t=m$ are obtained from any one solution by multiplying it on one side (whichever we please) by all the units; (ii) if $N t=m_{1}$ and $N u=m_{2}$, where $m_{1} \mid d$ and $m_{2} \mid d$, then $t u$ is divisible by the g.c.d. $m_{3}$ of $m_{1}$ and $m_{2}$, and $N\left(t u / m_{3}\right)=m_{1} m_{2} / m_{3}{ }^{2}$; (iii) the number of divisors $m$ of $d$, for which there exist quaternions of norm $m$, is a power of 2 , 
say $2^{h}$; (iv) if the number $w$ of units is finite, then adj $f$ has $2^{h-1} w$ positive, integral automorphs, and $F$ has $2^{h-1} w^{2}$ positive, integral automorphs.

Proof. (i) By Lemma 10, $u=\bar{t} \theta$, where $u$ and $t$ are any two quaternions of norm $m$. (ii) We can suppose $f \equiv a_{1} x_{1}{ }^{2}+a_{2} x_{2}{ }^{2}+p a_{3} x_{3}{ }^{2} \bmod p^{2}$, for any odd $p$ in $d$, where $\left(-a_{1} a_{2} \mid p\right)=-1$ and $p \nmid a_{3}$; and that if $d \equiv 2 \bmod 4, f \equiv x_{1}{ }^{2}+x_{1} x_{2}$ $+x_{2}{ }^{2}+2 \mu x_{3}{ }^{2} \bmod 8, \mu$ odd. Hence if $N t \equiv 0 \bmod p, t_{0} \equiv t_{3} \equiv 0 \bmod p$. Suppose $N t \equiv N u \equiv 0(\bmod p)$. If $p>0$, then $t u \equiv\left(i_{1} t_{1}+i_{2} t_{2}\right)\left(i_{1} u_{1}+i_{2} u_{2}\right) \equiv 0(\bmod p)$, since $i_{1}{ }^{2} \equiv i_{2}{ }^{2} \equiv i_{1} i_{2} \equiv i_{2} i_{1} \equiv 0 \bmod p$. If $p=2$, the same result is easily verified; for example, by (1) of $\S 1$,

$$
\begin{aligned}
(t u)_{0}+(t u)_{3} / 2= & t_{0}^{\prime} u_{0}^{\prime}-2 \mu t_{1} u_{1}-\mu\left(t_{1} u_{2}+t_{2} u_{1}\right)-2 \mu t_{2} u_{2}-3 t_{3} u_{3} / 4 \\
& +\left(t_{0}^{\prime} u_{3}+t_{3} u_{0}+2 \mu\left(t_{1} u_{2}-t_{2} u_{1}\right)\right) / 2 \\
= & t_{0} u_{0}+t_{0} u_{3}+t_{3} u_{0}-2 \mu\left(t_{1} u_{1}+t_{2} u_{2}\right) \equiv 0(\bmod 2), \\
(t u)_{1}= & t_{0}^{\prime} u_{1}+t_{1}^{\prime} u_{0}+1\left(t_{2} u_{3}-t_{3} u_{2}\right)+\left(t_{3} u_{1}-t_{1} u_{3}\right) / 2 \\
= & t_{0} u_{1}+t_{1} u_{0}+t_{3} u_{1}+t_{2} u_{3}-t_{3} u_{2} \equiv 0(\bmod 2),
\end{aligned}
$$

and so on. It follows that if $N t=m_{1}$, and $N u=m_{2}$, then $t u$ has $m_{3}$ as divisor. Since $d$ is squarefree, $t u / m_{3}$ can have no further rational integer divisor, and (ii) follows. (iii) is an easy consequence of (ii), and (iv) now follows from Theorem 4.

At this point we draw attention to Theorem 8 , which can also be verified (though less simply) by the preceding methods. Indeed, one finds, whether adj $f$ is fundamental or not, that $t x u / m$ is integral for all integral $x$ if and only if $t x \bar{t} / m$ is purely-integral for all purely-integral $x$.

8. The connection between $f$ and $F$. We now proceed more easily:

Theorem 7. If $F=\left(x_{0}+2^{-1} \sum \epsilon_{\alpha} x_{\alpha}\right)^{2}+\operatorname{adj} f$ is carried into $G$ $=\left(x_{0}+2^{-1} \sum \eta_{\alpha} x_{\alpha}\right)^{2}+\operatorname{adj} g$ by an integral transformation, then adj $f$ is carried into adj $g$ by an integral transformation. In particular, if $F \sim G$, then $\operatorname{adj} f \sim \operatorname{adj} g$, whence $f \sim \pm g$.

For, if $A$ and $B$ denote the matrices of $\operatorname{adj} f$ and $\operatorname{adj} g$, then $A_{1}$ and $B_{1}$ are integral matrices, where $A_{1}=A+\epsilon \epsilon^{\prime} / 4, B_{1}=B+\eta \eta^{\prime} / 4$. Any integral transformation of $F$ into $G$ is expressible in the form

$$
\left[\begin{array}{cc}
t_{0} & \tau^{\prime} \\
\sigma & T^{\prime}
\end{array}\right]\left[\begin{array}{ll}
1 & \epsilon^{\prime} / 2 \\
\epsilon / 2 & A_{1}
\end{array}\right]\left[\begin{array}{ll}
t_{0} & \sigma^{\prime} \\
\tau & T
\end{array}\right]=\left[\begin{array}{ll}
1 & \eta^{\prime} / 2 \\
\eta / 2 & B_{1}
\end{array}\right],
$$

where $\sigma^{\prime}=\left(s_{1}, s_{2}, s_{3}\right), \tau^{\prime}=\left(t_{1}, t_{2}, t_{3}\right)$, and $T$ are integral matrices. The expansion of (1) gives the following three equations:

$$
\begin{aligned}
\left(t_{0}+\tau^{\prime} \epsilon / 2\right)^{2}+\tau^{\prime} A \tau & =1, \\
\left(t_{0}+\tau^{\prime} \epsilon / 2\right)\left(\sigma^{\prime}+\epsilon^{\prime} T / 2\right)+\tau^{\prime} A T & =\eta^{\prime} / 2, \\
\left(\sigma+T^{\prime} \epsilon / 2\right)\left(\sigma^{\prime}+\epsilon^{\prime} T / 2\right)+T^{\prime} A T & =B+\eta \eta^{\prime} / 4,
\end{aligned}
$$


the transpose of (3) being $\left(\sigma+T^{\prime} \epsilon / 2\right)\left(t_{0}+\epsilon^{\prime} \tau / 2\right)+T^{\prime} A \tau=\eta / 2$.

Hence if $t=t_{0}+\sum j_{\alpha} t_{\alpha}, N t=1$ (in the set of quaternions related to $F$ ) and the coordinates of $t$ form the first column of the transformation replacing $F$ by $G$. Now $y=t x$ (where $y=y_{0}+\sum j_{\alpha} y_{\alpha}, x=x_{0}+\sum j_{\alpha} x_{\alpha}$ ) is evidently an automorphic transformation of $F$, since $N t=1$ and $N y=N x$; and the first column, giving the coefficients of $x_{0}$, also consists of the coordinates of $t$. The inverse of this transformation multiplied by the transformation applied to $F$ in (1) produces another transformation replacing $F$ by $G$, which has $t_{0}=1$, and $t_{1}=t_{2}=t_{3}=0$. Supposing this to be the transformation employed at the start, we find that (3) and (4) imply $\sigma^{\prime}+\epsilon^{\prime} T / 2=\eta^{\prime} / 2, \sigma+T^{\prime} \epsilon / 2=\eta / 2$,

$$
T^{\prime} A T=B \text {. }
$$

Thus $T$ is a transformation replacing adj $f$ by adj $g$, and the theorem follows.

Suppose now that $F=G$, so that (1) defines an automorph of $F$. The preceding process associates with every integral automorph of $F$ a uniquely determined integral automorph of $\operatorname{adj} f$, satisfying, that is,

$$
T^{\prime} A T=A,
$$

and a uniquely determined quaternion $t$ of norm 1 . Conversely, if $T$ is any integral solution of (6), the matrix

$$
\left[\begin{array}{cc}
1 & \epsilon^{\prime}(I-T) / 2 \\
0 & T
\end{array}\right]
$$

is an integral automorph of $F$. For if we put $t_{0}=1, \tau=0$, we see that equations (2)-(4) are satisfied with $\sigma=\left(I-T^{\prime}\right) \epsilon / 2$. Also, $\sigma$ is integral, since by (1) of $\$ 3,4 A \equiv \epsilon \epsilon^{\prime} \bmod 2, T^{\prime}(4 A) T=4 A, T^{\prime} \epsilon \epsilon^{\prime} T \equiv \epsilon \epsilon^{\prime}$; hence if $T^{\prime} \epsilon=\zeta$, $\zeta_{\alpha}{ }^{2} \equiv \epsilon_{\alpha}{ }^{2}(\alpha=1,2,3)$, or $\epsilon \equiv T^{\prime} \epsilon \bmod 2$. Finally, if $t$ is any unit quaternion, the automorph $y=t x$ multiplied by that in (7) produces any desired integral automorph of $F$. We have thus proved the following theorem.

THEOREM 8. The number of integral automorphs of $F$ is equal to the number. of integral automorphs of $f$ multiplied by the number of units.

In particular, if the only units are \pm 1 , every integral automorph of $F$ is given by. (7) or its negative, where $T$ ranges over the integral automorphs of $\operatorname{adj} f$. This case occurs when $f$ is definite and the minimum of adj $f$ exceeds 1 .

The definite forms $f$ for which adj $f$ has minimum $3 / 4$ or 1 will be determined in the next section. At the same time, for later use, we shall isolate also the forms for which adj $f$ has minimum $7 / 4$ or 2 .

THEOREM 9. Every form $G$ of minimum 1 in the genus of a norm-form $F$ is equivalent to a norm-form belonging to a ternary $g$ in the genus of $\pm f$.

For $G$ is equivalent to a form $\left(x_{0}+2^{-1} \sum \eta_{\alpha} x_{\alpha}\right)^{2}+\sum B_{\alpha \beta} x_{\alpha} x_{\beta}$, where the $\eta_{\alpha}$ 
are 0 or 1 , and $B+\eta \eta^{\prime} / 4$ is an integral matrix. Comparison of determinants gives $|B|=|A|$. Since $F$ and $G$ are in the same genus, (1) holds with $t_{0}, \sigma, \tau$, and $T$ having rational elements with denominators prime to $2 d$. Now $y=t x$ determines a rational transformation of denominators prime to $2 d$, with $t$ in the first column. Proceeding as before, we get (5) with $T$ rational and of denominator prime to $2 d$. Hence $4 A$ and $4 B$ are in the same genus, and the theorem follows.

9. The positive, integral forms $f$ such that adj $f$ has minimum not greater than 2.

(i) First suppose that $\xi^{\prime} A \xi$ represents $3 / 4$. By a unimodular transformation we can take $A_{33}=3 / 4$. Hence $a_{11} x_{1}{ }^{2}+2 a_{12} x_{1} x_{2}+a_{22} x_{2}{ }^{2}$ becomes an integral positive binary form of determinant $3 / 4$. All such forms are equivalent to $x_{1}{ }^{2}+x_{1} x_{2}+x_{2}{ }^{2}$, and we can therefore assume $a_{11}=2 a_{12}=a_{22}=1$. Next by a translation on $x_{3}$ in adj $f$, we can obtain $\left|2 A_{13}\right| \leqq 3 / 4,\left|2 A_{23}\right| \leqq 3 / 4$. Hence $A_{13}=\alpha_{1} / 4, A_{23}=\alpha_{2} / 4$, where $\alpha_{1}=0$ or $\pm 1, \alpha_{2}=0$ or \pm 1 . The matrices of $f$ and $\operatorname{adj} f$ now have the following appearance:

$$
\left[\begin{array}{ccc}
1 & 1 / 2 & \cdot \\
1 / 2 & 1 & \cdot \\
\cdot & \cdot & \cdot
\end{array}\right], \quad \frac{1}{4}\left[\begin{array}{ccc}
4 A_{11} & 4 A_{12} & \alpha_{1} \\
4 A_{21} & 4 A_{22} & \alpha_{2} \\
\alpha_{1} & \alpha_{2} & 3
\end{array}\right] .
$$

Let $d=4\left|a_{\alpha \beta}\right|$. Then adj adj $f=d f / 4$, and in particular,

$$
4 d=12 A_{11}-\alpha_{1}{ }^{2}, \quad 2 d=\alpha_{1} \alpha_{2}-12 A_{12}, \quad 4 d=12 A_{22}-\alpha_{2}{ }^{2} .
$$

Hence $\alpha_{1}{ }^{2} \equiv \alpha_{2}{ }^{2} \equiv-2 \alpha_{1} \alpha_{2} \bmod 3$, and we can suppose either $\alpha_{1}=\alpha_{2}=0$, or $\alpha_{1}=\alpha_{2}=-1$ (the signs of both $\alpha_{1}$ and $\alpha_{2}$ can evidently be changed without affecting $\left(1_{1}\right)$ ). In these two cases, $d=3 k-1$ or $3 k$, where $k$ is a positive integer, and $\operatorname{adj} f$ has the respective matrices

$$
\frac{1}{4}\left[\begin{array}{ccc}
4 k-1 & 1-2 k & -1 \\
1-2 k & 4 k-1 & -1 \\
-1 & -1 & 3
\end{array}\right], \quad \frac{1}{4}\left[\begin{array}{ccc}
4 k & -2 k & 0 \\
-2 k & 4 k & 0 \\
0 & 0 & 3
\end{array}\right]
$$

the corresponding forms $f$ are as follows:

$$
x_{1}{ }^{2}+x_{2}{ }^{2}+k x_{3}{ }^{2}+x_{1} x_{2}+x_{1} x_{3}+x_{2} x_{3}, \quad x_{1}{ }^{2}+x_{1} x_{2}+x_{2}{ }^{2}+k x_{3}{ }^{2} .
$$

(ii) Suppose that adj $f$ has minimum 1. We can take $A_{38}=1$. Hence $a_{11} x_{1}^{2}+2 c_{12} x_{1} x_{2}+a_{22} x_{2}{ }^{2}$ can be taken to be $x_{1}{ }^{2}+x_{2}{ }^{2}$. By a translation we get $\left|2 A_{13}\right| \leqq 1,\left|2 A_{23}\right| \leqq 1$. Hence $A_{13}=\alpha_{1} / 4, A_{23}=\alpha_{2} / 4$, where $\alpha_{1}, \alpha_{2}=0, \pm 1$, or \pm 2 . Since the coefficient of $x_{1} x_{2}$ in $f$ is 0 , the negative $\alpha_{i}$ can be dropped. We have (1) with 0 in place of each $1 / 2$ in the upper left, and 4 in place of the 3 on the lower right. Hence

$$
4 d=16 A_{11}-\alpha_{1}{ }^{2}, \quad 4 d=16 A_{22}-\alpha_{2}{ }^{2}, \quad 0=\alpha_{1} \alpha_{2}-16 A_{12} ;
$$


$\alpha_{1}{ }^{2} \equiv \alpha_{2}{ }^{2} \equiv \alpha_{1} \alpha_{2} \equiv 0(\bmod 4)$. We can set $\alpha_{i}=2 \beta_{i}$, where $\beta_{i}=0$ or 1 . Then $A_{11}=\left(d+\beta_{1}{ }^{2}\right) / 4, A_{22}=\left(d+\beta_{2}{ }^{2}\right) / 4, A_{12}=\beta_{1} \beta_{2} / 4$, and

$$
\begin{gathered}
F=\left(x_{0}+2^{-1} \sum \epsilon_{\alpha} x_{\alpha}\right)^{2}+\left(d+\beta_{1}{ }^{2}\right) x_{1}{ }^{2} / 4+\left(d+\beta_{2}{ }^{2}\right) x_{2}{ }^{2} / 4 \\
+x_{3}{ }^{2}+\beta_{1} x_{1} x_{3}+\beta_{2} x_{2} x_{3}+\beta_{1} \beta_{2} x_{1} x_{2} / 2 .
\end{gathered}
$$

Since $F$ must have integral coefficients, $\epsilon_{3}=0, \epsilon_{1} \epsilon_{2}+\beta_{1} \beta_{2}$ is even and $\epsilon_{1}{ }^{2}+\beta_{1}{ }^{2}$ $\equiv \epsilon_{2}{ }^{2}+{\beta_{2}}^{2} \bmod 4$. This implies that $\epsilon_{1}=\beta_{2}, \epsilon_{2}=\beta_{1}$, and $d \equiv-\epsilon_{1}{ }^{2}-\epsilon_{2}{ }^{2} \bmod 4$. The forms $f$ obtained from $\left(\epsilon_{1}, \epsilon_{2}\right)=(0,1)$ and $(1,0)$ are equivalent. There remain three forms $f$ :

(6) $x_{1}{ }^{2}+x_{2}{ }^{2}+k x_{3}{ }^{2}-x_{2} x_{3}-x_{3} x_{1}, x_{1}{ }^{2}+x_{2}{ }^{2}-x_{1} x_{3}+k x_{3}{ }^{2}, x_{1}{ }^{2}+x_{2}{ }^{2}+k x_{3}{ }^{2}$,

and $d$ is respectively $4 k-2,4 k-1$, and $4 k$. We can suppose $k \geqq 1$ in connection with $\left(6_{3}\right), k \geqq 2$ in $\left(6_{1}\right)$ and $\left(6_{2}\right)$. For, if $k=1$ in $\left(6_{1}\right)$ or $\left(6_{2}\right)$, adj $f$ represents $3 / 4$, and it is seen that $f$ is equivalent respectively to the case $k=1$ of $\left(4_{1}\right)$ and $\left(4_{2}\right)$.

(iii) Let adj $f$ have minimum $7 / 4$. As before we readily obtain:

$$
\left[\begin{array}{ccc}
1 & 1 / 2 & \cdot \\
1 / 2 & 2 & \cdot \\
\cdot & \cdot & \cdot
\end{array}\right], \quad \frac{1}{4}\left[\begin{array}{ccc}
4 A_{11} & 4 A_{12} & \alpha_{1} \\
4 A_{21} & 4 A_{22} & \alpha_{2} \\
\alpha_{1} & \alpha_{2} & 7
\end{array}\right] ;
$$

where $\alpha_{1}, \alpha_{2}=0, \pm 1, \pm 2, \pm 3 ; 4 A_{11}=\left(\alpha_{1}{ }^{2}+8 d\right) / 7, \quad 4 A_{22}=\left(\alpha_{2}{ }^{2}+4 d\right) / 7$, $4 A_{12}=\left(\alpha_{1} \alpha_{2}-2 d\right) / 7 ;-4 \alpha_{1} \alpha_{2} \equiv \alpha_{1}{ }^{2} \equiv 2 \alpha_{2}{ }^{2}(\bmod 7) ;\left(\alpha_{1}, \alpha_{2}\right)=(0,0)$ and $d=7 k$, $\left(\alpha_{1}, \alpha_{2}\right)=(1,-2)$ and $d=7 k-1,\left(\alpha_{1}, \alpha_{2}\right)=(-2,-3)$ and $d=7 k-4$, or $\left(\alpha_{1}, \alpha_{2}\right)=(-3,-1)$ and $d=7 k-2$. Hence we have four forms, with $d$ respectively $7 k-4,7 k-2,7 k-1$, and $7 k$, and each demanding $k \geqq 2$ :

$$
\begin{gathered}
x_{1}{ }^{2}+2 x_{2}{ }^{2}+k x_{3}{ }^{2}+x_{1} x_{2}+x_{1} x_{3}+2 x_{2} x_{3}, \\
x_{1}{ }^{2}+2 x_{2}{ }^{2}+k x_{3}{ }^{2}+x_{2} x_{3}+x_{3} x_{1}+x_{1} x_{2}, \\
x_{1}{ }^{2}+2 x_{2}{ }^{2}+k x_{3}{ }^{2}+x_{1} x_{2}+x_{2} x_{3}, \quad x_{1}{ }^{2}+2 x_{2}{ }^{2}+k x_{3}{ }^{2}+x_{1} x_{2} .
\end{gathered}
$$

(iv) Let adj $f$ have the minimum 2. Then we have

$$
\left[\begin{array}{ccc}
1 & 0 & \cdot \\
0 & 2 & \cdot \\
\cdot & \cdot & \cdot
\end{array}\right], \quad \frac{1}{4}\left[\begin{array}{ccc}
4 A_{11} & 4 A_{12} & \alpha_{1} \\
4 A_{21} & 4 A_{22} & \alpha_{2} \\
\alpha_{1} & \alpha_{2} & 8
\end{array}\right] ;
$$

$\alpha_{1}, \alpha_{2}=0,1,2,3$, or $4 ; 4 A_{11}=\left(\alpha_{1}{ }^{2}+8 d\right) / 8,4 A_{22}=\left(\alpha_{2}{ }^{2}+4 d\right) / 8, \alpha_{1} \alpha_{2}=32 A_{12}$; $\alpha_{1}=4 \beta_{1}, \alpha_{2}=2 \beta_{2}, \beta_{1}=0$ or $1, \beta_{2}=0,1$, or $2 ; \epsilon_{3}=0$,

$$
\left(x_{0}+\epsilon_{1} x_{1} / 2+\epsilon_{2} x_{2} / 2\right)^{2}+\left(2 \beta_{1}{ }^{2}+d\right) x_{1}{ }^{2} / 4+\left({\beta_{2}}^{2}+d\right) x_{2}{ }^{2} / 8
$$

$$
+2 x_{3}^{2}+\beta_{1} \beta_{2} x_{1} x_{2} / 2+2 \beta_{1} x_{1} x_{3}+\beta_{2} x_{2} x_{3}
$$

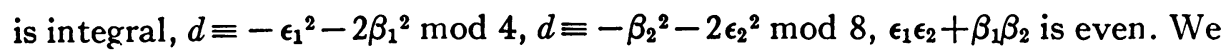


thus have six cases: (a) $d=8 k-6, \epsilon_{1}=0, \epsilon_{2}=1, \beta_{1}=1, \beta_{2}=2$; (b) $d=8 k-4$, $\epsilon_{1}=\epsilon_{2}=\beta_{1}=0, \beta_{2}=2$; (c) $d=8 k-3, \epsilon_{1}=\epsilon_{2}=\beta_{1}=\beta_{2}=1$; (d) $d=8 k-2, \epsilon_{1}=0$, $\epsilon_{2}=1=\beta_{1}, \beta_{2}=0$; (e) $d=8 k-1, \epsilon_{1}=1, \epsilon_{2}=0=\beta_{1}, \beta_{2}=1$; (f) $d=8 k, \epsilon_{1}=\epsilon_{2}=\beta_{1}$ $=\beta_{2}=0$. The corresponding forms $f$ are respectively:

$$
\begin{array}{ll}
x_{1}^{2}+2 x_{2}{ }^{2}+k x_{3}{ }^{2}-x_{1} x_{3}-2 x_{2} x_{3}(k \geqq 3), & x_{1}{ }^{2}+2 x_{2}{ }^{2}+k x_{3}{ }^{2}-2 x_{2} x_{3}(k \geqq 2), \\
x_{1}{ }^{2}+2 x_{2}{ }^{2}+k x_{3}{ }^{2}-x_{1} x_{3}-x_{2} x_{3}(k \geqq 3), & x_{1}{ }^{2}+2 x_{2}{ }^{2}+k x_{3}{ }^{2}-x_{1} x_{3}(k \geqq 3), \\
x_{1}{ }^{2}+2 x_{2}{ }^{2}+k x_{3}{ }^{2}-x_{2} x_{3}(k \geqq 2), & x_{1}{ }^{2}+2 x_{2}{ }^{2}+k x_{3}{ }^{2}(k \geqq 2) .
\end{array}
$$

10. Problem; to find all the fundamental definite norm-forms $F$ for which factorization, as of Theorem 3, is always possible. The genus of a fundamental norm-form represents all positive in tegers. Hence $F$ must represent 2 , and so must belong to one of the forms in (4), (6), (7), and (9) of $\$ 9$. We number these in order, $1^{\circ}$ to $15^{\circ}$. Note also that $d$ must be squarefree (excluding $5^{\circ}, 11^{\circ}$, and $\left.15^{\circ}\right)$, and $c_{p}$ is -1 for each $p$ in $d$. Since $f$ is definite this implies that $d$ contains an odd number of primes.

$$
\begin{aligned}
& 1^{\circ} \text {. Then } F=\left(x_{0}+x_{1} / 2+x_{2} / 2+x_{3} / 2\right)^{2}+\phi / 4, \text { where } \\
& \phi=3 x_{3}{ }^{2}+(4 k-1) x_{2}{ }^{2}+(4 k-1) x_{1}{ }^{2}-(4 k-2) x_{1} x_{2}-2 x_{1} x_{3}-2 x_{2} x_{3} .
\end{aligned}
$$

Since $\phi$ is Eisenstein-reduced if $k \geqq 1$, the least number primitively represented by $\phi$ with $\left(x_{1}, x_{2}\right) \neq(0,0)$ is $4 k-1$. Since $F\left(x_{0}, 0,0, x_{3}\right)=x_{0}{ }^{2}+x_{0} x_{3}+x_{3}{ }^{2}$ $\neq 2, F$ does not represent 2 if $k \geqq 3$.

There remain the cases $k=1$ and 2 , that is, $d=2$ and 5 , when $F$ is indeed fundamental and in a genus of one class (Theorem 10).

$2^{\circ}$. $F=x_{0}{ }^{2}+x_{0} x_{3}+x_{3}{ }^{2}+k\left(x_{1}{ }^{2}+x_{1} x_{2}+x_{2}{ }^{2}\right)$, and $F \neq 2$ if $k \geqq 3$. If $k=2, d=6$ and contains two primes. There remains $k=1$, or $d=3$ (Theorem 10).

$3^{\circ} . F=\left(x_{0}+x_{1} / 2+x_{2} / 2\right)^{2}+\phi / 4, \quad \phi=4 x_{3}{ }^{2}+(4 k-1) x_{2}{ }^{2}+(4 k-1) x_{1}{ }^{2}+2 x_{1} x_{2}$ $+4 x_{1} x_{3}+4 x_{2} x_{3}$. Hence if $k \geqq 4, F \neq 3$. Since $c_{p}=(2-4 k,-1)_{p}, c_{2}=(-1)^{k}$; and if $k=3, c_{5}=1$. Hence no fundamental $F$ remains, as $k \geqq 2$.

$4^{\circ}$. $F=x_{0}{ }^{2}+x_{0} x_{2}+k x_{2}{ }^{2}+x_{3}{ }^{2}+x_{3} x_{1}+k x_{1}{ }^{2}$. If $k \geqq 4, F \neq 3$. If $k=3$, we might point to the fact that $f=(1,1,3,0,-1 / 2,0)$ is not alone in its genus, being accompanied by $g=(1,1,4,1 / 2,1 / 2,1 / 2)$. (For further information on $f$, see $\left[8\right.$, p. 173].) Hence $G=\left(x_{0}+2^{-1} \sum x_{\alpha}\right)^{2}+\left(15 x_{1}{ }^{2}+15 x_{2}{ }^{2}+3 x_{3}{ }^{2}-14 x_{1} x_{2}\right.$ $\left.-2 x_{1} x_{3}-2 x_{2} x_{3}\right) / 4$ is in the same genus as $F$; and since $G \neq 2, G$ and $F$ are inequivalent. However, although this proves that $F$ is not in a genus of one class, it does not prove (since $G$ represents 1 ) that factorization, as of Theorem 3, may fail. For this reason, we point to the following third form in the genus, of minimum 2 :

$$
\begin{aligned}
2 x_{0}{ }^{2}+2 x_{1}{ }^{2}+2 x_{2}{ }^{2}+ & 2 x_{3}{ }^{2}+x_{0} x_{1}-x_{0} x_{2}+2 x_{0} x_{3}-2 x_{1} x_{2}-x_{2} x_{3} \\
=2( & \left.x_{0}+x_{1} / 4-x_{2} / 4+x_{3} / 2\right)^{2} \\
& +\left(12 x_{3}{ }^{2}+15 x_{2}{ }^{2}-15 x_{1}{ }^{2}-14 x_{1} x_{2}-4 x_{1} x_{3}-4 x_{2} x_{3}\right) / 8,
\end{aligned}
$$

whence indeed factorization must sometimes fail. 
There remains the case $k=2, d=7$ (Theorem 10 ).

$6^{\circ} . F=\left(x_{0}+x_{2} / 2+x_{3} / 2\right)^{2}+\phi / 4, \phi=7 x_{3}{ }^{2}+(4 k-1) x_{2}{ }^{2}+(8 k-4) x_{1}{ }^{2}$ $-(4 k-4) x_{1} x_{2}-4 x_{1} x_{3}-6 x_{2} x_{3}$. Hence as $F\left(x_{0}, 0,0, x_{3}\right)=x_{0}^{2}+x_{0} x_{3}+2 x_{3}^{2} \neq 3, F$ does not represent 3 if $(4 k-1) / 4>3$, or $k \geqq 4$. If $k=2, d=10$. Finally, if $k=3$, whence $d=17$, then besides another norm-form in the genus corresponding to $g=(1,1,6,1 / 2,1 / 2,1 / 2)$, there is the following form of minimum 2 in the genus of $F$ :

$$
\begin{array}{r}
2\left(x_{0}+x_{1} / 4-x_{2} / 4+x_{3} / 2\right)^{2}+\left(23 x_{1}{ }^{2}+23 x_{2}{ }^{2}+12 x_{3}{ }^{2}-4 x_{2} x_{3}-4 x_{1} x_{3}-22 x_{1} x_{2}\right) / 8 \\
=2 x_{0}^{2}+3 x_{1}{ }^{2}+3 x_{2}{ }^{2}+2 x_{3}{ }^{2}+x_{0} x_{1}-x_{0} x_{2}+2 x_{0} x_{3}-3 x_{1} x_{2}-x_{2} x_{3} .
\end{array}
$$

70. $F=\left(x_{0}+x_{1} / 2+x_{2} / 2+x_{3} / 2\right)^{2}+\phi / 4, \quad \phi=(8 k-1) x_{1}{ }^{2}+(4 k-1) x_{2}{ }^{2}+7 x_{3}{ }^{2}$ $-(2 k-1) x_{1} x_{2}-3 x_{1} x_{3}-x_{2} x_{3}$. If $k=2, d=12$. If $k \geqq 4, F \neq 3$. If $k=3$, the genus of $F$ contains the following form of minimum 2:

$$
\begin{array}{r}
2\left(x_{0}+x_{1} / 4-x_{2} / 2\right)^{2}+\left(23 x_{1}{ }^{2}+20 x_{2}{ }^{2}+16 x_{3}{ }^{2}+4 x_{1} x_{2}+16 x_{1} x_{3}+8 x_{2} x_{3}\right) / 8 \\
=2 x_{0}^{2}+3 x_{1}^{2}+2 x_{2}{ }^{2}+2 x_{3}{ }^{2}+x_{0} x_{1}-2 x_{0} x_{2}+2 x_{1} x_{3}+x_{2} x_{3} .
\end{array}
$$

$8^{\circ} . F=\left(x_{0}+x_{1} / 2+x_{3} / 2\right)^{2}+\phi / 4, \phi=(8 k-1) x_{1}^{2}+4 k x_{2}{ }^{2}+7 x_{3}{ }^{2}+4 k x_{1} x_{2}+2 x_{1} x_{3}$ $+4 x_{2} x_{3}$. Hence if $k \geqq 4, F \neq 3$. If $k=3, d=20$.

If $k=2, d=13$, and $F$ is in a genus of one class (Theorem 10).

$9^{\circ}$. $F=x_{0}{ }^{2}+x_{0} x_{3}+2 x_{3}{ }^{2}+k\left(2 x_{1}{ }^{2}+x_{1} x_{2}+x_{2}{ }^{2}\right)$. Hence if $k \geqq 4, F \neq 3$. If $k=2$ or $3, d=14$ or 21 , and $F$ is not fundamental.

$10^{\circ} . F=\left(x_{0}+x_{2} / 2\right)^{2}+\phi / 4, \phi=(8 k-4) x_{1}{ }^{2}+(4 k-1) x_{2}{ }^{2}+8 x_{3}{ }^{2}+4 x_{1} x_{2}+8 x_{1} x_{3}$ $+8 x_{2} x_{3}$. Since $d=8 k-6, c_{2}=1$ if $k$ is even. If $k=3$ or $5, d=18$ or 34 . If $k \geqq 7$, $F\left(x_{0}, 0,0, x_{3}\right)=x_{0}^{2}+2 x_{3}^{2} \neq 5$, and $(4 k-1) / 4>5 ; F \neq 5$.

12。. $F=\left(x_{0}+x_{1} / 2+x_{2} / 2\right)^{2}+\phi / 4, \phi=(8 k-1) x_{1}{ }^{2}+(4 k-1) x_{2}{ }^{2}+8 x_{3}{ }^{2}+2 x_{1} x_{2}$ $+8 x_{1} x_{3}+4 x_{2} x_{3}$. If $k \geqq 6, F \neq 5$. If $k=3, d=21$. But if $k=4$ or $5, d=29$ or 37 , we have again to construct forms of minimum 2 in the genus of $F$ :

$$
\begin{array}{r}
2\left(x_{0}+x_{2} / 4-x_{3} / 4\right)^{2}+\left(32 x_{1}{ }^{2}+31 x_{2}{ }^{2}+15 x_{3}{ }^{2}+16 x_{1} x_{2}+8 x_{1} x_{3}+2 x_{2} x_{3}\right) / 8 \\
=2 x_{0}{ }^{2}+4 x_{1}{ }^{2}+4 x_{2}{ }^{2}+2 x_{3}{ }^{2}+x_{0} x_{2}-x_{0} x_{3}+2 x_{1} x_{2}+x_{1} x_{3} ; \\
2\left(x_{0}+x_{1} / 4+x_{2} / 4-x_{3} / 4\right)^{2}+\left(39 x_{1}{ }^{2}+31 x_{2}{ }^{2}+23 x_{3}{ }^{2}-10 x_{1} x_{2}-6 x_{1} x_{3}-22 x_{2} x_{3}\right) / 8 \\
=2 x_{0}{ }^{2}+5 x_{1}{ }^{2}+4 x_{2}{ }^{2}+3 x_{3}{ }^{2}+x_{0} x_{1}+x_{0} x_{2}-x_{0} x_{3}-x_{1} x_{2}-x_{1} x_{3}-3 x_{2} x_{3} .
\end{array}
$$

13 $3^{\circ} F=x_{0}{ }^{2}+x_{0} x_{2}+k x_{2}{ }^{2}+2\left(x_{3}{ }^{2}+x_{3} x_{1}+k x_{1}{ }^{2}\right)$. Here $c_{2}$ is 1 unless $k$ is odd. If $k=3$ or $5, d=22$ or 38 . If $k \geqq 7, F \neq 5$.

$14^{\circ}$. $F=x_{0}{ }^{2}+x_{0} x_{1}+2 k x_{1}{ }^{2}+k x_{2}{ }^{2}+x_{2} x_{3}+2 x_{3}{ }^{2}$. If $k=2$ or $5, c_{3}=1$. If $k \geqq 6$, $F \neq 5$. But if $k=3$ or $4, d=23$ or 31 , we need the following forms of minimum 2 in the genus of $F$ :

$$
2 x_{0}{ }^{2}+x_{0} x_{1}+k x_{1}{ }^{2}+2 x_{2}{ }^{2}+x_{2} x_{3}+k x_{3}{ }^{2} .
$$

Summing up, we can state the following theorem:

TheOREM 10. The only fundamental definite norm-forms in whose (maximal) 
quaternion arithmetics factorization is always possible are the following:

$$
\begin{array}{lrl}
F_{2}=x_{0}{ }^{2}+x_{1}{ }^{2}+x_{2}{ }^{2}+x_{3}{ }^{2}+x_{0} x_{1}+x_{0} x_{2}+x_{0} x_{3}, & \text { where } d=2 ; \\
F_{3}=x_{0}{ }^{2}+x_{0} x_{3}+x_{3}{ }^{2}+x_{1}{ }^{2}+x_{1} x_{2}+x_{2}{ }^{2}, & d=3 ; \\
F_{5}=x_{0}{ }^{2}+x_{1}{ }^{2}+2 x_{2}{ }^{2}+2 x_{3}{ }^{2}+x_{0} x_{1}+x_{0} x_{2}+x_{0} x_{3}-x_{1} x_{2}, & d=5 ; \\
F_{7}=x_{0}{ }^{2}+x_{0} x_{2}+2 x_{2}{ }^{2}+x_{3}{ }^{2}+x_{3} x_{1}+2 x_{1}{ }^{2}, & d=7 ; \\
F_{13}=x_{0}{ }^{2}+4 x_{1}{ }^{2}+2 x_{2}{ }^{2}+2 x_{3}{ }^{2}+x_{0} x_{1}+x_{0} x_{3}+2 x_{1} x_{2}+x_{1} x_{3}+x_{2} x_{3}, & d=13 .
\end{array}
$$

To complete the proof we must show that each of these five forms is in a genus of one class. This can be done by means of formulae for the weight of a genus, making use of the number of automorphs. (See references to Smith in $\$ \S 13$ and 14.) This is possible since, the determinant being a square, the weight of the quaternary genus can be expressed in a finite form. And indeed this method was used by the author in 1941, but not published, to obtain 37 norm-forms in genera of one class, two of the forms in Theorem 11 being overlooked. This was not as good a result as we have now, since it left open the possibility of a genus containing several classes, which all have minimum 1.

A result of Korkine and Zolotareff [9] shows that $a^{4} \leqq 4 \delta$, where $a$ is the minimum and $\delta$ is the determinant of a definite quaternary form $G$. In the present case $\delta=d^{2} / 16$. Hence $a=1$ for the genera of $F_{2}, F_{3}, F_{5}$, and $F_{7}$; but $a$ is 1 or 2 , for the genus of $F_{13}$. Now the ternaries $f$ corresponding to the five $F_{d}$ are easily seen to be in genera of one class. It rema ns only to shcw that there is no form of minimum 2 in the genus of $F_{13}$.

If the minimum is 2 we can take $G=2 x_{0}^{2}+x_{0}\left(a_{1} x_{1}+a_{2} x_{2}+a_{3} x_{3}\right)+\cdots$. If all the $a_{\alpha}$ could be even, the determinant of $G$ would be either half or quarter of an integer; but the determinant is $169 / 16$. Hence we can suppose, that the g.c.d. of the $a_{\alpha}$ is 1 , and can replace (through the inverse of a unimodular transformation) $\sum a_{\alpha} x_{\alpha}$ by $x_{1}$. We thus obtain

$$
G=2 x_{0}^{2}+x_{0} x_{1}+\cdots=2\left(x_{0}+x_{1} / 4\right)^{2}+\phi\left(x_{1}, x_{2}, x_{3}\right) / 8,
$$

whence $\phi \equiv 7 x_{1}{ }^{2}(\bmod 8)$. Indeed, $\phi$ is equivalent to a form congruent $\bmod 2^{r}$ to $7 y_{1}^{2}+8 y_{2} y_{3}$, since $1 \equiv \operatorname{det}(2 G) \equiv(-1)(-1) \bmod 8$. Also, the form-residue $\bmod 13^{r}$ of $F$ shows that $\phi \sim \nu z_{1}{ }^{2}+13\left(z_{2}{ }^{2}+\nu z_{3}{ }^{2}\right) \bmod 13^{r}$, where $\nu$ is a quadratic non-residue $\bmod 13$. Since $\operatorname{det} \phi=52^{2}$, we have adj $\phi=52 \psi$, where $\psi$ is an improperly primitive form of determinant 52 , and adj $\psi=\phi$. The minimum $a$ of $\psi$ satisfies $a^{3} \leqq 104, a=2$ or 4 . If $a=2$, then $3 a^{2} / 4 \leqq C \leqq(416 / 3)^{1 / 2}, 3 \leqq C \leqq 11$; since $C$ must be represented by $\phi, C$ may be 7 or 8 (which agree with the form-residues of $\phi$ above). But if $\phi$ represents 7 (necessarily with $x_{1}$ odd), $G=2\left(x_{0}+x_{1} / 4\right)^{2}+\phi / 8$ evidently represents 1 ; if $\phi$ represents $8,4 \mid x_{1}$, and $G$ again represents 1 . If $a=4,12 \leqq C \leqq(832 / 3)^{1 / 2}<17$. The only $C$ consistent with the form-residues of $\phi$ is $C=15$. But $\phi\left(0, x_{2}, x_{3}\right)=B x_{2}{ }^{2}+2 R x_{2} x_{3}+C x_{3}{ }^{2}$, 
where $B C-R^{2}=4.52$; and this is impossible since $(-208 \mid 3)=-1$. Hence there is no form in the genus of minimum 2 . The reader will discern here the essentials of the method used to get forms of minimum 2 in $\$ 10$.

11. Diagonal forms for the arithmetics of $F_{2}, \cdots, F_{13}$. We saw in $\S 1$ that the system of integral quaternions related to $F_{2}$ can be carried by the transformation (5) of $\$ 1$ into the system $\Sigma_{2}$ :

$$
\left(y_{0}+i_{1} y_{1}+i_{2} y_{2}+i_{3} y_{3}\right) / 2, \quad y_{0} \equiv y_{1} \equiv y_{2} \equiv y_{3}(\bmod 2),
$$

the $y_{i}$ integers, the $i_{\alpha}$ pertaining to $(1,1,1)$.

Clearly, any system of integral quaternions can be transformed into infinitely many such diagonal systems. It is only necessary to transform the norm-form into a form $Y_{0}^{2}+a_{2} a_{3} Y_{1}{ }^{2}+a_{3} a_{1} Y_{2}{ }^{2}+a_{1} a_{2} Y_{3}{ }^{2}$, where the $Y_{i}$ are linear expressions with rational coefficients in the $x_{i}$, and to work out the conditions of integrality.

For example, consider $F_{5}$. Since adj $f$ has $c_{5}=-1$, and $c_{p}$ is +1 for all other $p$, we seek a form $\left(a_{2} a_{3}, a_{3} a_{1}, a_{1} a_{2}\right)$ of a small determinant, with the same property, so that it shall be rationally equivalent to adj $f$. We easily find $(5,10,2)$. Now 4 adj $f=7 x_{1}{ }^{2}+7 x_{2}{ }^{2}+3 x_{3}{ }^{2}-6 x_{1} x_{2}-2 x_{1} x_{3}-2 x_{2} x_{3}$, and has determinant 100, and cannot be expressed as 5()$^{2}+10()^{2}+2()^{2}$, where the indicated linear forms are to have integer coefficients. We therefore take the next best, $16 \operatorname{adj} f$, and find the expression

$$
5\left(-2 x_{2}\right)^{2}+10\left(x_{1}-x_{3}\right)^{2}+2\left(3 x_{1}-2 x_{2}+x_{3}\right)^{2} .
$$

We therefore obtain a norm-form $\left(y_{0} / 2\right)^{2}+5\left(y_{1} / 2\right)^{2}+10\left(y_{2} / 4\right)^{2}+2\left(y_{3} / 4\right)^{2}$, by (2) $y_{0}=2 x_{0}+x_{1}+x_{2}+x_{3}, y_{1}=-x_{2}, y_{2}=x_{1}-x_{3}, y_{3}=3 x_{1}-2 x_{2}+x_{3}$.

The $y_{i}$ are then integers with the $x_{i}$, but the integrity of the $x_{i}$ requires $y_{2}+y_{3} \equiv 2 y_{1}(\bmod 4), y_{0}+y_{1} \equiv y_{2}(\bmod 2)$. Hence the system of integral quaternions associated with $F_{5}$ becomes transformed into $\Sigma_{5}$ :

$$
\begin{aligned}
& \left(2 y_{0}+2 y_{1} i_{1}+y_{2} i_{2}+y_{3} i_{3}\right) / 4, \\
& y_{0}+y_{1} \equiv y_{2}(\bmod 2), \quad y_{2}+y_{3} \equiv 2 y_{1}(\bmod 4),
\end{aligned}
$$

the $y_{i}$ integers, the $i_{\alpha}$ pertaining to $f=(2,1,5)$. That is, for the last, $i_{1}{ }^{2}=-5$, $i_{2}{ }^{2}=-10, i_{3}{ }^{2}=-2, i_{1} i_{2}=-i_{2} i_{1}=5 i_{3}$, and so on.

In a similar way, using the respective transformations

(4) $y_{0}=2 x_{0}+x_{1}, y_{1}=x_{1}, y_{2}=x_{2}, y_{3}=x_{2}+2 x_{3}$, for $F_{3}$ and $F_{7}$,

(5) $y_{0}=2 x_{0}+x_{1}+x_{3}, y_{1}=+x_{1}, y_{2}=-x_{3}, y_{3}=2 x_{1}+4 x_{2}+x_{3}$, for $F_{13}$, we find for $F_{3}, F_{7}$, and $F_{13}$ the arithmetics $\Sigma_{3}, \Sigma_{7}$, and $\Sigma_{13}$ :

$$
\left(y_{0}+i_{1} y_{1}+i_{2} y_{2}+i_{3} y_{3}\right) / 2, \quad y_{0} \equiv y_{1}, y_{2} \equiv y_{3}(\bmod 2),
$$

the $y_{i}$ integers, the $i_{\alpha}$ pertaining to $f=(1,1,3)$ for $\Sigma_{3}$, to $(1,1,7)$ for $\Sigma_{7}$; 


$$
\begin{aligned}
&\left(2 y_{0}+2 y_{1} i_{1}+y_{2} i_{2}+y_{3} i_{3}\right) / 4 \\
& y_{0}+y_{1} \equiv y_{2}(\bmod 2), y_{2}+y_{3} \equiv 2 y_{1}(\bmod 4),
\end{aligned}
$$

the $i_{\alpha}$ pertaining to $f=(2,1,13)$ for $\Sigma_{13}$.

The author wishes here to acknowledge the valuable assistance of Miss C. S. Williams, who checked most of the computations in this and the following sections.

The preceding diagonal forms will simplify the work of deriving the nonmaximal systems in which, subject to (1) of $\$ 5$, factorization is always possible.

12. Integral transformations, especially of norm-forms into norm-forms. Two integral matrices $T_{1}, T_{2}$ are called right-equivalent if there exists a unimodular matrix $U$ such that $T_{1}=T_{2} U$. H. J.S. Smith [13, vol. I, p. 389] has shown that any integral matrix of order $r$ and determinant $n(>0)$ is rightequivalent to a unique matrix

$$
\left[\begin{array}{cccc}
n_{1} & n_{12} & \cdots & n_{1 r} \\
0 & n_{2} & \cdots & n_{2 r} \\
\cdot & \cdot & \cdot & \cdot \\
0 & 0 & \cdots & n_{r}
\end{array}\right],
$$$$
n=n_{1} \cdots n_{r}, 0 \leqq n_{i j}<n_{i}
$$

Hermite was the first to give a general enunciation of this [6(b), p. 192]. Hence, if $n$ is a prime $p$, an integral matrix of order 3 is right-equivalent to one and only one of the $p^{2}+p+1$ prime matrices

$$
\left[\begin{array}{lll}
p & \alpha & \beta \\
0 & 1 & 0 \\
0 & 0 & 1
\end{array}\right],\left[\begin{array}{lll}
1 & 0 & 0 \\
0 & p & \alpha \\
0 & 0 & 1
\end{array}\right],\left[\begin{array}{lll}
1 & 0 & 0 \\
0 & 1 & 0 \\
0 & 0 & p
\end{array}\right], \quad \alpha, \beta=0,1, \cdots, p-1 .
$$

We shall now prove the following lemma.

LEMma 14. Let $T$ be an integral matrix of determinant $n$. Then for every expression $n=p_{1} p_{2} \cdots p_{2}$ as a product of primes in some order, we can find prime matrices $P_{1}, \cdots, P_{s}$ such as in (2) and of respective determinants $p_{1}, \cdots, p_{0}$, and a unimodular matrix $U$, such that

$$
T=P_{1} P_{2} \cdots P_{8} U \text {. }
$$

It is easily seen that $T$ can be expressed in this form with the primes $p_{i}$ in some particular order. For example,

$$
\left[\begin{array}{lll}
1 & 0 & 0 \\
0 & p n & \alpha \\
0 & 0 & m
\end{array}\right]=\left[\begin{array}{lll}
1 & 0 & 0 \\
0 & n & \alpha \\
0 & 0 & m
\end{array}\right]\left[\begin{array}{lll}
1 & 0 & 0 \\
0 & p & 0 \\
0 & 0 & 1
\end{array}\right]
$$


and unit factors can eventually be moved to the extreme right, by Smith's result. It remains only to show that if $P$ and $Q$ are of determinants $p$ and $q$, where $p$ and $q$ are distinct primes, then $P Q=Q_{1} P_{1} U_{1}$. We can suppose $P$ and $Q$ to be of the types in (2); hence $P Q$ is of one of the forms

$$
\left[\begin{array}{lll}
p q & \alpha & \beta \\
0 & 1 & 0 \\
0 & 0 & 1
\end{array}\right], \quad\left[\begin{array}{lll}
p & \alpha & \beta \\
0 & q & \gamma \\
0 & 0 & 1
\end{array}\right], \quad\left[\begin{array}{lll}
p & \alpha & \beta \\
0 & 1 & 0 \\
0 & 0 & q
\end{array}\right], \quad\left[\begin{array}{lll}
q & \alpha & \beta \\
0 & p & \gamma \\
0 & 0 & 1
\end{array}\right],
$$

and so on. In the first three cases the diagonal matrix $\{p, 1,1\}$ can obviously be factored out to the right. In the fourth case,

$$
\left[\begin{array}{ccc}
q & \alpha & \beta \\
0 & p & \gamma \\
0 & 0 & 1
\end{array}\right]=\left[\begin{array}{lll}
1 & n_{2} & 0 \\
0 & 1 & 0 \\
0 & 0 & 1
\end{array}\right] \cdot\left[\begin{array}{ccc}
q & 0 & \beta-n_{2} \gamma \\
0 & p & \gamma \\
0 & 0 & 1
\end{array}\right] \cdot\left[\begin{array}{ccc}
1 & n_{1} & 0 \\
0 & 1 & 0 \\
0 & 0 & 1
\end{array}\right],
$$

where $n_{1}$ and $n_{2}$ are integers such that $\alpha=n_{2} p+n_{1} q$. Clearly, $\{1, p, 1\}$ can be factored out at the right of the middle matrix.

We consider now the conditions under which a norm-form $G$ is derivable from a norm-form $F$ by applying an integral transformation $T$ of determinant $n$ to the variables $x_{\alpha}$, that is, to $\operatorname{adj} f$, and then a translation on $x_{0}$ (to make the coefficients of $x_{0} x_{\alpha}$ be 0 or 1 . If $A=\left(A_{\alpha \beta}\right)=\operatorname{adj}\left(a_{\alpha \beta}\right)$, where $\left(a_{\alpha \beta}\right)$ is semiintegral, the matrix $T^{\prime} A T$ will not, in general (if $n>1$ ), be the adjoint of a semi-integral matrix $\left(b_{\alpha \beta}\right)$. If $T^{\prime} A T$ is the adjoint of some semi-integral matrix, we shall call $T$ a suitable transformation for $A$.

If $T$ is suitable, and $T^{\prime} A T=\operatorname{adj}\left(b_{\alpha \beta}\right)$, then comparing determinants we get $n^{2} \Delta^{2}=\left|b_{\alpha \beta}\right|^{2}$, and we can choose the sign of $\left(b_{\alpha \beta}\right)$ to get $\left|b_{\alpha \beta}\right|=n \Delta$. Hence if $U$ is the matrix of cofactors of $T$, whence $U^{\prime} T=T U^{\prime}=U T^{\prime}=n I$, then

$$
U^{\prime}\left(a_{\alpha \beta}\right) U=n\left(b_{\alpha \beta}\right) \text {. }
$$

Conversely, (4) implies $T^{\prime} A T=\operatorname{adj}\left(b_{\alpha \beta}\right)$. Hence: $a$ necessary and sufficient condition that $T$ be suitable for $A$ is that $U^{\prime}\left(a_{\alpha \beta}\right) U$ be "divisible by $n$," the quotient to be semi-integral.

If $n=p^{2} m$, where $p$ is a prime not dividing $m$, and if $T$ is suitable, then $T_{1}$ must be suitable, if we factor $T$ as $T_{1} T_{2},\left|T_{1}\right|=p^{*},\left|T_{2}\right|=m, T_{1}$ and $T_{2}$ integral. For if (in obvious notations)

$$
U_{2}^{\prime} U_{1}^{\prime}\left(a_{\alpha \beta}\right) U_{1} U_{2}=p^{8} m\left(b_{\alpha \beta}\right),
$$

where $\left(b_{\alpha \beta}\right)$ is semi-integral, then multiplying by $T_{2}$ and $T_{2}{ }^{\prime}$, we get

$$
U_{1}^{\prime}\left(a_{\alpha \beta}\right) U_{1}=p^{\prime}\left(T_{2}\left(b_{\alpha \beta}\right) T_{2}^{\prime} / m\right) .
$$

Since the leftside is semi-integral, the right side must be likewise, and since $m$ is prime to $p, T_{2}\left(b_{\alpha \beta}\right) T_{2}{ }^{\prime}$ must be "divisible by $m . "$ 
Lemma 15. If $|T|=p^{s}(s \geqq 1)$, and if $T$ is suitable for $A$, then $T$ has $a$ left-divisor of determinant $p$ which is suitable for $A$, except that (i) if $d$ is prime to $p$, and (ii) if $f \sim a_{1} x_{1}{ }^{2}+a_{2} x_{2}{ }^{2}+a_{3} x_{3}{ }^{2}\left(\bmod p^{r}\right)$, where $p>2, p \mid a_{3}$, and $\left(-a_{1} a_{2} \mid p\right)=-1$, or if $f \sim x_{1}{ }^{2}+x_{1} x_{2}+x_{2}{ }^{2}+\kappa x_{3}{ }^{2}\left(\bmod 2^{r}\right)$, where $p=2$ and $\kappa$ is even, then $T$ may not have a suitable left-divisor of determinant $p$, but if so will have one of determinant $p^{2}$.

Using Hermite's result we can suppose that

$$
T=\left[\begin{array}{lll}
p^{\rho} & \lambda & \mu \\
0 & p^{\sigma} & \nu \\
0 & 0 & p^{\tau}
\end{array}\right], \quad \begin{aligned}
& 0 \leqq \lambda, \mu<p^{p}, 0 \leqq \nu<p^{\sigma}, \\
& \rho+\sigma+\tau=s, \rho, \sigma, \tau \geqq 0 .
\end{aligned}
$$

We shall actually prove a little more in case (ii), namely that if we take the $\sim$ as being $\equiv$ (as we can, by a unimodular transformation), then any matrix which has no suitable left-divisor of determinant $p$ will be right-equivalent to a matrix (5) where $\rho>0, \sigma>0, \tau=0, \lambda \equiv 0 \bmod p$, and then

$$
P_{\mu \nu}^{\prime}=\left[\begin{array}{lll}
p & 0 & \mu \\
0 & p & \nu \\
0 & 0 & 1
\end{array}\right]
$$

is a left-divisor of $T$, and is suitable for $A$.

If $f$ has the form $a_{1} x_{1}{ }^{2}+a_{2} x_{2}{ }^{2}+a_{3} x_{3}{ }^{2}$ mod $p^{r}$, then the three diagonal elements of $U^{\prime}\left(a_{\alpha \beta}\right) U$, and the doubles of the three non-diagonal elements, are:

$$
\begin{aligned}
& p^{2 \rho+2 \sigma} a_{3}, p^{2 \rho+2 \tau} a_{2}+p^{2 \rho} \nu^{2} a_{3}, p^{2 \sigma+2 \tau} a_{1}+p^{2 \tau} \lambda^{2} a_{2}+\left(\lambda \nu-p^{\sigma} \mu\right)^{2} a_{3} ; \\
& -2 p^{2 \rho+\sigma} \nu a_{3}, 2 p^{\rho+\sigma}\left(\lambda \nu-p^{\sigma} \mu\right) a_{3},-2 \dot{p}^{\rho+2 \tau} \lambda a_{2}-2 p^{\rho} \nu\left(\lambda \nu-p^{\sigma} \mu\right) a_{3} .
\end{aligned}
$$

The condition for $T$ to be suitable is that $p^{e}$ divide these six numbers.

If $p$ divides every $a_{\alpha}$, evidently every matrix of determinant $p$ is suitable. If $p$ divide $a_{2}$ and $a_{3}$ but not $a_{1}$, then if $T$ is suitable, $\sigma+\tau>0$; if now $\tau>0$, the diagonal matrix $P_{p}=\{1,1, p\}$ is a suitable left-divisor of $T$; and if $\tau=0$ but $\sigma>0$, the matrix

$$
P_{\nu}=\left[\begin{array}{lll}
1 & 0 & 0 \\
0 & p & \nu \\
0 & 0 & 1
\end{array}\right]
$$

is a suitable left-divisor of $T$. Hence assume $p \nmid a_{1} a_{2}$. Also, let $p \mid a_{3}$. The matrices $P_{p}$ and $P_{\mu \nu}{ }^{\prime}$ are suitable. If we left-multiply $T$ in (5) by $P_{p}{ }^{-1}$ we get an integral product if $\tau \geqq 1$. Hence let $\tau=0$. Then the suitability of $T$ implies by $\left(7_{2}\right)$ that $\rho>0$.

Now assume $p>2$. Then either $\sigma>0$ and $p \mid \lambda$ (by $\left(8_{3}\right)$ ), or $\sigma=0$ and $p \mid a_{1}+a_{2} \lambda^{2}$ (by $\left.\left(7_{3}\right)\right)$. In the last case, $\nu=0$ and 


$$
P_{\lambda \mu}=\left[\begin{array}{lll}
p & \lambda & \mu \\
0 & 1 & 0 \\
0 & 0 & 1
\end{array}\right]
$$

is a suitable left-divisor of $T$. But if $\rho>0, \sigma>0$, and $p \mid \lambda$, then $P_{\mu \nu}{ }^{\prime}$ is a suitable left-divisor of $T$. Further, $P_{\mu \nu}{ }^{\prime}$ has no suitable left-divisor of determinant $p$ if $\left(-a_{1} a_{2} \mid p\right)=-1$, and has $P_{\lambda \mu}$ as a left-divisor if $p \mid a_{1}+a_{2} \lambda^{2}$.

Similarly, let $p=2$. Then by $\left(7_{3}\right)$, either $\sigma=0$ and $\lambda$ is odd, or $\sigma>0$ and $\lambda$ is even. In the first case, $P_{\lambda_{\mu}}$ is a suitable left-divisor of $T$. In the second case $P_{\mu \nu}{ }^{\prime}$ is a suitable left-divisor of $T$, and has $P_{1, \mu \rightarrow \nu}$ as a suitable left-divisor.

Next assume that $p\left\{a_{1} a_{2} a_{3}, p>2\right.$. It can be verified that the matrix $P_{\lambda \mu}$ is suitable if $p \mid a_{1}+a_{2} \lambda^{2}+a_{3} \mu^{2}, P_{\nu}$ is suitable if $p \mid a_{2}+a_{3} \nu^{2}$; and that the three matrices of determinant $p^{2}$,

$$
P_{p}^{\prime}=\left[\begin{array}{lll}
1 & 0 & 0 \\
0 & p & 0 \\
0 & 0 & p
\end{array}\right], \quad P_{\lambda}^{\prime}=\left[\begin{array}{lll}
p & \lambda & 0 \\
0 & 1 & 0 \\
0 & 0 & p
\end{array}\right], \quad P_{\mu_{111}{ }^{\prime}},
$$

are suitable. In connection with (11) we make the respective assumptions

$$
\left(-a_{2} a_{3} \mid p\right)=-1, \quad p \nmid a_{1}+a_{2} \lambda^{2}, \quad p \nmid a_{1} a_{2}+a_{2} a_{3} \mu_{1}^{2}+a_{3} a_{1} \nu_{1}^{2} .
$$

For, if $\left(12_{1}\right)$ does not hold, $P_{p}^{\prime}$ is left-divisible by $P_{\nu}$; if $\left(12_{2}\right)$ does not hold, $P_{\lambda}{ }^{\prime}$ is left-divisible by $P_{\lambda 0}$. Lastly, $P_{\nu}$ will be a left-divisor of $P_{\mu_{1 \nu_{1}}}{ }^{\prime}$ if $p \mid a_{2}+a_{3} \nu^{2}$, excluded by $\left(12_{3}\right)$ if $p \mid \mu_{1}$. Again, $P_{\lambda \mu}$ will be a left-divisor of $P_{\mu_{11}}{ }^{\prime}$ if and only if $\lambda$ and $\mu$ satisfy

$$
a_{1}+a_{2} \lambda^{2}+a_{3} \mu^{2} \equiv 0, \quad \mu_{1}-\lambda \nu_{1}-\mu \equiv 0(\bmod p) .
$$

Eliminating $\mu$, and assuming that $p \mid a_{1} a_{2}+a_{2} a_{3} \mu_{1}{ }^{2}+a_{3} a_{1} \nu_{1}{ }^{2}$, we readily obtain $\left(a_{1} \nu_{1}+a_{2} \mu_{1} \lambda\right)^{2} \equiv 0(\bmod p)$, which is solvable for $\lambda$ if $p \nmid \mu_{1}$.

Now $T$ has $P_{p}^{\prime}$ as a left-divisor if $\sigma \geqq 1, \tau \geqq 1$, and $p \mid \nu$. (i) Suppose that $\sigma=0$, whence $\nu=0$. Since $p^{\rho+\tau} \mid\left(7_{1}\right),\left(8_{2}\right)$, and $\left(7_{3}\right)$, we have $\tau \leqq \rho, \mu=p^{\tau} \mu_{1}$, $p^{\rho-\tau} \mid a_{1}+a_{2} \lambda^{2}+a_{3} \mu_{1}^{2}$. If here $\rho=\tau$, then as $0 \leqq \mu<p^{\rho}, \mu=0$ and $P_{\lambda \mu}$ is a leftdivisor of $T$. But if $\rho>\tau$, then $P_{\lambda_{\mu 1}}$ is a left-divisor. (ii) Suppose $\sigma \geqq 1, \tau=0$. If $p \mid a_{2}+a_{3} \nu^{2}, P_{\nu}$ is a left-divisor of $T$. Assume $p \nmid a_{2}+a_{3} \nu^{2}$. Then $\sigma \leqq \rho$ by $\left(7_{2}\right)$, $\lambda=p^{0} \lambda_{1}$ by $\left(8_{3}\right), p^{\rho-\sigma} \mid a_{1}+a_{2} \lambda_{1}{ }^{2}+a_{3}\left(\lambda_{1} \nu-\mu\right)^{2}$ by $\left(7_{3}\right)$. If now $\rho=\sigma$, whence $\lambda=0$, then $P_{\mu \nu}{ }^{\prime}$ is a left-divisor of $T$. But if $\rho>\sigma$, then $P_{\lambda_{1}, \mu-\lambda_{1} \nu}$ is a leftdivisor. (iii) Finally suppose $\sigma \geqq 1, \tau \geqq 1, p \nmid \nu$. By $\left(8_{1}\right), \tau \leqq \rho$. If now $\sigma \leqq \tau$, then by $\left(8_{3}\right), p^{\sigma+r} \mid \lambda \nu-p^{\sigma} \mu, \lambda=p^{\sigma} \lambda_{1}, \lambda_{1}$ an integer, and $p \mid \lambda_{1} \nu-\mu$. And if $\sigma>\tau$, then by $\left(8_{2}\right), \lambda=p^{\tau} \lambda_{2}$; by $\left(8_{3}\right), p^{\sigma}\left|\lambda_{2}\left(a_{2} p^{2 \tau}+a_{3} \nu^{2}\right)-p^{\sigma-\tau} a_{3} \mu \nu, p^{\sigma-\tau}\right| \lambda_{2}$, ' $\lambda=p^{\sigma} \lambda_{1}, p^{\tau}\left|a_{3} \nu\left(\lambda_{1} \nu-\mu\right), p\right| \lambda_{1} \nu-\mu$. In either case, $P_{\lambda_{1}}$ ' is a left-divisor of $T$.

Now assume $p \nmid a_{1} a_{2} a_{3}, p=2$. The suitability of $T$ implies (i) $\rho+\sigma>0$; (ii) $\rho>0$, or $\rho=0$ and $2 \mid 2^{\tau}+\nu$; (iii) $\tau>0$ and $2 \mid \lambda \nu-2 \sigma \mu$, or $\tau=0$ and $2 \mid 2^{\sigma}+\lambda+\lambda \nu-2^{\sigma} \mu$. Hence, if $\rho=0$, then $\sigma>0, \lambda=\mu=0$, and $P$, is a suitable 
left-divisor of $T$. If $\rho>0$, and $\sigma=\tau=0$, then $\nu=0$ and $\lambda+\mu$ is odd; hence $P_{\lambda \mu}$ is a suitable left-divisor. If $\rho>0$ and $\tau=0<\sigma$, then $2 \mid \lambda(1+\nu)$; then $P_{\lambda_{1} \mu_{1}}$ is a left-divisor if $\lambda$ is even, and $\mu-\lambda_{1} \nu-\mu_{1}$ even, and is suitable if $\lambda_{1}+\mu_{1}$ is odd: this can be achieved when $\lambda$ is even unless $\nu$ is odd. Also, if $\nu$ is odd, $P_{\nu}$ is a suitable left-divisor. Lastly, if $\rho>0$ and $\tau>0$, then $2 \mid \lambda \nu-2{ }^{\sigma} \mu$, and $P_{2}$ succeeds.

We assume next that $f \equiv j x_{1}{ }^{2}+x_{1} x_{2}+j x_{2}{ }^{2}+(j+1) \gamma x_{3}{ }^{2}, j=0$ or $1, \gamma$ an integer. Then $2^{s}$ must divide the six numbers

$$
\begin{array}{cc}
(j+1) 2^{2 \rho+2 \sigma} \gamma, j 2^{2 \rho+2 \tau}+(j+1) 2^{2 \rho} \gamma \nu^{2}, 2^{2 \sigma+2 \tau} j-2^{\sigma+2 \tau} \lambda+2^{2 \tau} \lambda^{2} j \\
& +(j+1) \gamma\left(\lambda \nu-2^{\sigma} \mu\right)^{2} ; \\
(j+1) 2^{2 \rho+\sigma+1} \gamma \nu,(j+1) 2^{\rho+\sigma+1} \gamma\left(\lambda \nu-2^{\sigma} \mu\right), & 2^{\rho+\sigma+2 \tau}-j \lambda 2^{\rho+2 \tau+1} \\
& -(j+1) 2^{\rho+1} \gamma \nu\left(\lambda \nu-2^{\sigma} \mu\right) .
\end{array}
$$

(Note also that if $f$ is replaced by $2^{\delta} f, \delta>0$, these six numbers are multiplied by $2^{\delta}$, and every matrix of determinant 2 is suitable.)

Let $\gamma$ be odd. The suitable matrices of determinant 2 or 4 , omitting some of determinant 4 which have suitable left-divisors of determinant 2 , are $P_{2}$ and $P_{\mu_{111}}{ }^{\prime}$ if $j=1$, and the following if $j=0$ :

$$
P_{2}{ }^{*}=\left[\begin{array}{ccc}
1 & 0 & 0 \\
0 & 2 & 0 \\
0 & 0 & 1
\end{array}\right], \quad P_{\lambda_{1} \lambda_{1}}=\left[\begin{array}{ccc}
2 & \lambda_{1} & \lambda_{1} \\
0 & 1 & 0 \\
0 & 0 & 1
\end{array}\right], \quad P_{1,1}{ }^{\prime}=\left[\begin{array}{lll}
2 & 0 & 1 \\
0 & 2 & 1 \\
0 & 0 & 1
\end{array}\right] \text {. }
$$

Now let $j=0$. Evidently $P_{2}{ }^{*}$ is a left-divisor of $T$ if $\sigma \geqq 1$ and $\nu$ is even; $P_{\lambda_{1} \lambda_{1}}$ is a left-divisor (for $\lambda_{1}$ zero or one) if $\rho>0, \sigma=0, \mu \equiv 2^{2 \tau} \lambda \bmod 2$; or if

$$
\rho>0, \sigma>0, \lambda \text { is even, } \nu \text { is odd, } 2^{\tau} \mu \text { is even; }
$$

likewise for $P_{1,1}{ }^{\prime}$ if

$$
\rho>0, \sigma>0, \lambda \text { is even, } \nu \text { is odd, } 2^{\tau} \mu \text { is odd. }
$$

Further, if $\sigma=0$ (whence $\nu=0$ ) and $T$ is suitable, then by (13), $2^{\rho+\tau} \mid-2^{2 \tau} \lambda$ $+\gamma \mu^{2}, \mu \equiv 2^{2 \tau} \lambda \bmod 2$. Hence let $\sigma>0$ and $\nu$ be odd. Then by $\left(13_{2}\right), \rho \geqq \sigma+\tau>0$, and by $\left(13_{3}\right), \lambda$ is even; and either (16) or (15) holds.

Now let $j=1$. Then $P_{2}$ is a left-divisor if and only if $\tau \geqq 1$; and if $\tau=0$, $P_{\mu_{1 \nu_{1}}}$ is a left-divisor of $T$ (for some choice of $\mu_{1}, \nu_{1}=0$ or 1 ) if and only if $\rho \geqq 1, \lambda$ is even, $\mu$ is even if $\tau>0, \sigma \geqq 1, \nu$ is even if $\tau>0$. But if $T$ is suitable and $\tau=0$, then $\left(13_{3}\right)$ shows that $\sigma \geqq 1$ and $\lambda$ is even, $\left(13_{2}\right)$ that $\rho \geqq \sigma$. Thus in all cases $P_{2}$ or $P_{\mu 1 \nu 1}{ }^{\prime}$ is a divisor.

Let $\gamma$ be even. If $\tau>0, P_{2}$ is a suitable divisor. If $\tau=0, \sigma>0$, and $j=0$, then $P_{\nu}$ is effective. If $j=1$ and $\tau=0$, then if $T$ is suitable, $\rho>0$ by $\left(13_{2}\right)$, $\sigma>0$ by $\left(13_{3}\right), \lambda$ is even by $\left(13_{3}\right)$, and $P_{\mu \nu}{ }^{\prime}$ is a left-divisor of $T$ and has no 
suitable left-divisors of determinant 2. If $j=0, \tau=0=\sigma$, then $\rho>0$, and $\lambda$ must be even by $\left(13_{3}\right)$, and $P_{\lambda \mu}$ succeeds.

Finally, let $f \equiv 2^{\alpha} a_{1} x_{1}{ }^{2}+2^{\beta+2}\left(j x_{2}{ }^{2}+x_{2} x_{3}+j x_{3}{ }^{2}\right)$, where $\ddot{j}=0$ or $1, a_{1}$ is odd, and $\alpha \leqq \beta$. If $\alpha>0$ every matrix of determinant 2 is suitable. Let $\alpha=0$. If $T$ is suitable, $\sigma+\tau>0$. If $\tau>0$, use $P_{2}$; if $\tau=0$ and $\sigma>0$, use $P_{\text {. }}$.

It follows from these lemmas that if a norm-form $G$ arises from a normform $F$ by an integral transformation of determinant $n$, then a form equivalent to $G$ can be arrived at by a succession of transformations of type (5). And further we can use the prime factors of $n$ in any desired order, starting with a transformation of determinant $p$ or $p^{2}$, in most cases continuing with transformations of determinant $p$, and obtaining norm-forms at each step.

Suppose that at some step a norm-form $F_{1}$ is obtained for which a particular factorization with the properties in Theorem 3 is not possible. Then the genus of $F_{1}$ must contain a form $G_{1}$ of minimum greater than 1 . (Note that if an indefinite norm-form represents -1 , then by composition with itself it represents +1.) If we now apply a further integral transformation to $F_{1}$ to obtain $G$, then the same transformation replaces $G_{1}$ by a form $G_{2}$ in the genus of $G$. Since $G_{1}$ represents all the numbers represented by $G_{2}$, the minimum of $G_{2}$ is also greater than 1 . Hence we need seek no further for genera containing norm-forms only. All this will be illustrated and applied in $\S \S 13-15$.

13. Norm-forms derived from $F_{d}$ by transformations of determinant 2 . We shall now investigate the genera which: (a) contain norm-forms $G$, (b) are derived from $F_{2}$ by integral transformations of determinant a power of 2 , and (c) contain no classes of minimum greater than 1 . We shall find that there are exactly ten such genera, including that of $F_{2}$, and that each contains only one class.

We have first a lemma, which follows easily from Lemma 12, giving a form-residue $\bmod 2^{r}$ of any norm-form:

LEMMA 16. If $G$ is a norm-form corresponding to an integral ternary form $g$, then $G$ is equivalent to a form with one of the following residues mod $2^{r}, r$ large:

$$
\begin{gathered}
x_{0}{ }^{2}+a_{2} a_{3} x_{1}{ }^{2}+a_{3} a_{1} x_{2}{ }^{2}+a_{1} a_{2} x_{3}{ }^{2} ; \\
x_{0}{ }^{2}+x_{0} x_{3}+j x_{3}{ }^{2}+\lambda\left(j x_{1}{ }^{2}+x_{1} x_{2}+j x_{2}{ }^{2}\right) ; \\
x_{0}{ }^{2}+(4 j-1) 2^{2 \beta} x_{3}{ }^{2}+\lambda\left(j x_{1}{ }^{2}+x_{1} x_{2}+j x_{2}{ }^{2}\right) .
\end{gathered}
$$

Here $j=0$ or $1, \lambda$ and the $a_{\alpha}$ are integers, $\beta$ is a non-negative integer; if $j=1$ then $\lambda$ is even. Also, in (3), the power of 2 in $\lambda$ exceeds $2^{\beta}$ and must not equal $2^{2 \beta+1}$. In (3) the cases $j=0$ and 1 are equivalent mod $2^{r}$ if the power of 2 in $\lambda$ is $2^{2 \beta}$ or $2^{2 \beta+2}$. The values of $c_{2}$ are given by $c_{2}=\left(-a_{1} a_{2},-a_{1} a_{3}\right)_{2}$ in (1); and in (2) and (3) by

$$
\begin{aligned}
c_{2} & =(-1)^{j} \text { if } \lambda \text { contains an odd power of } 2 ; \\
& =+1 \quad \text { if } \lambda \text { contains an even power of } 2 .
\end{aligned}
$$


Since $G$ is now to be derived from $F_{2}$, we must have $c_{2}=-1$. Hence in both (2) and (3), $j=1$ and $\lambda$ contains an odd power of 2. Also, in (1), $\left(-a_{1} a_{2},-a_{1} a_{3}\right)_{2}$ must be -1 . Further we can replace $g$ by $m g, m$ odd, since this will not affect the form-residue of $G \bmod 2^{r}$; and so can suppose that the odd part of det $g$ is congruent to $1 \mathrm{mod} 8$. Hence an examination of the unique form-residues attainable in (1) for $g$ will show that if $\operatorname{det} g$ is $1,2,4$, or 8 , then $\left(a_{1}, a_{2}, a_{3}\right)$ can be taken to be one of the triples

$$
\begin{gathered}
(1,1,1) ;(1,1,2) ;(1,2,2),(1,1,4) ; \\
(2,2,2),(1,2,4),(1,1,8),(1,3,24),(-1,3,-24), \quad \bmod 2^{r} .
\end{gathered}
$$

I. Forms $G$ of determinant 1 . The only residue in (1), (2), and (3) consistent with $\operatorname{det} G=1$, and with $\lambda$ limited as above, is $x_{0}{ }^{2}+x_{1}{ }^{2}+x_{2}{ }^{2}+x_{3}{ }^{2}$ mod $2^{r}$. That is, there is only one genus of determinant 1 to be considered. Evidently this genus contains the form

$$
F_{4}=y_{0}{ }^{2}+y_{1}{ }^{2}+y_{2}{ }^{2}+y_{3}{ }^{2},
$$

and (as is well known) this is in a genus of one class. Note that $F_{4}$ is derivable from $F_{2}$ by the transformation $x_{1}=y_{2}+y_{3}, x_{2}=y_{3}+y_{1}, x_{3}=y_{1}+y_{2}, x_{0}=y_{0}-y_{1}$ $-y_{2}-y_{3}$, of determinant 2 .

II. Forms $G$ of determinant 4 . The possible genera are determined by the form-residues $x_{0}{ }^{2}+2 x_{1}{ }^{2}+2 x_{2}{ }^{2}+x_{3}{ }^{2}$ and $x_{0}{ }^{2}+x_{0} x_{3}+x_{3}{ }^{2}+8\left(x_{1}{ }^{2}+x_{1} x_{2}+x_{2}{ }^{2}\right)$, $\bmod$ $2 r$. These genera contain the forms

$$
\begin{aligned}
F_{8} & =y_{0}{ }^{2}+2 y_{1}{ }^{2}+2 y_{2}{ }^{2}+y_{3}{ }^{2}, \\
F_{8}^{\prime} & =y_{0}{ }^{2}+y_{1}{ }^{2}+3 y_{2}{ }^{2}+3 y_{3}{ }^{2}+y_{0} y_{1}+y_{0} y_{2}+y_{0} y_{3}-2 y_{2} y_{3},
\end{aligned}
$$

and each of these is easily seen to belong to a genus of one class (cf. determinant 64 below). Clearly $F_{8}$ is derived from $F_{4}$ by a transformation of determinant 2 , and $F_{8}{ }^{\prime}$ from $F_{2}$ by a transformation of determinant 4 .

III. Forms $G$ of determinant 16. There are three possible genera, corresponding to the form-residues

$$
\begin{gathered}
x_{0}{ }^{2}+2 x_{1}{ }^{2}+2 x_{2}{ }^{2}+4 x_{3}{ }^{2}, \quad x_{0}{ }^{2}+x_{1}{ }^{2}+4 x_{2}{ }^{2}+4 x_{3}{ }^{2}, \\
x_{0}{ }^{2}+3 x_{3}{ }^{2}+8\left(x_{1}{ }^{2}+x_{1} x_{2}+x_{2}{ }^{2}\right),
\end{gathered}
$$

$\bmod 2^{r}$.

Each genus contains only one class (cf. next case). Representatives of these classes are

$$
\begin{gathered}
F_{16}=y_{0}{ }^{2}+2 y_{1}{ }^{2}+2 y_{2}{ }^{2}+4 y_{3}{ }^{2}, \quad F_{16}{ }^{\prime}=y_{0}{ }^{2}+4 y_{1}{ }^{2}+4 y_{2}{ }^{2}+y_{3}{ }^{2}, \\
F_{16}{ }^{\prime \prime}=y_{0}{ }^{2}+3 y_{1}{ }^{2}+3 y_{2}{ }^{2}+3 y_{3}{ }^{2}-2 y_{1} y_{2}-2 y_{1} y_{3}-2 y_{2} y_{3} .
\end{gathered}
$$

IV. Forms $G$ of determinant 64 . There are six possible genera, corresponding to the form-residues

$$
\begin{gathered}
(1,4,4,4),(1,2,4,8),(1,1,8,8),(1,3,8,24), \\
(1,-3,-8,24), x_{0}{ }^{2}+x_{0} x_{3}+x_{3}{ }^{2}+32\left(x_{1}{ }^{2}+x_{1} x_{2}+x_{2}{ }^{2}\right), \bmod 2 r .
\end{gathered}
$$


The second, third, fourth, and sixth contain the following forms of minimum greater than 1 :

$2 y_{0}{ }^{2}+3 y_{1}{ }^{2}+2 y_{1} y_{2}+3 y_{2}{ }^{2}+4 y_{3}{ }^{2}$,

$2 y_{0}^{2}+5 y_{1}^{2}+5 y_{2}^{2}+2 y_{3}^{2}+2 y_{0} y_{1}-2 y_{0} y_{2}+2 y_{1} y_{3}+2 y_{2} y_{3}$,

$3\left(y_{0}+y_{1} / 3+y_{2} / 3-y_{3} / 3\right)^{2}+\left(11 y_{1}{ }^{2}+11 y_{2}{ }^{2}+8 y_{3}{ }^{2}+10 y_{1} y_{2}+8 y_{1} y_{3}+8 y_{2} y_{3}\right) / 3$,

$3\left(y_{0}+y_{1} / 3-y_{2} / 3+y_{3} / 2\right)^{2}+\left(44 y_{1}{ }^{2}+44 y_{2}{ }^{2}+27 y_{3}{ }^{2}-40 y_{1} y_{2}-12 y_{1} y_{3}-12 y_{2} y_{3}\right) / 12$.

However, the forms corresponding to $\left(11_{1}{ }^{\prime}\right)$ and $\left(11_{5}{ }^{\prime}\right)$,

$$
\begin{aligned}
& F_{32}=y_{0}{ }^{2}+4 y_{1}{ }^{2}+4 y_{2}{ }^{2}+4 y_{3}{ }^{2}, \\
& F_{32}{ }^{\prime}=y_{0}^{2}+5 y_{1}{ }^{2}+5 y_{2}{ }^{2}+4 y_{3}{ }^{2}+2 y_{1} y_{2}+4 y_{1} y_{3}+4 y_{2} y_{3},
\end{aligned}
$$

are in genera of one class. This may be seen, in the case of $F_{32}$, by the fact that the reciprocal $(1,1,1,4)$ of $F_{32}$, which will be found in various tables of quaternaries (the best, with determinant up to 25 , is Townes [14]); or by our methods of construction which when applied exhaustively lead to representatives of every class in a given genus; or, most simply, by H. J. S. Smith's explicit formula [13, vol. II, p. 666] for the weight of a genus. Thus in the case of $F_{32}{ }^{\prime}$, which has 16 positive automorphs, we have (in Smith's notations [13, vol. II, pp. 666-668]) $I_{1}=1, I_{2}=8, I_{3}=1, W=(1 / 12) \zeta(1 / 2)^{0}(1 / 2)^{0} 8^{2}\left(1 / \pi^{2}\right)\left(\pi^{2} / 8\right)$ $=(2 / 3) \zeta$, where $\zeta=(3 / 128)(3-1)(3-1)=3 / 32, W=1 / 16$; hence $F_{32}{ }^{\prime}$ is in a genus of one class.

V. Forms $G$ of determinant 256. Neither (11) nor (12), nor any of the residues mod $2^{r}$ in case III, are of the special forms in (i) or (ii) of Lemma 15. Hence we have only to consider the genera derived from (11) and (12) by suitable transformations of determinant 2. Every transformation (on the $y_{\alpha}$ ) of determinant 2 is suitable for (11), and we get three derived genera, represented by

$$
(1,4,4,16),(1,4,8,8), x_{0}{ }^{2}+48 \dot{x}_{3}{ }^{2}+8\left(x_{1}{ }^{2}+x_{1} x_{2}+x_{2}{ }^{2}\right), \bmod 2^{r}
$$

from $(1,24,-8,-3)$, whence $a_{1}=1, a_{2}=-3, a_{3}=-8$, we find by the suitable transformations $P_{2}, P_{1, \mu}$ the two form-residues

$$
(1,24,-8,-12), \quad(1,-48,16,-3),
$$

$\bmod 2 r$

The genus of $\left(13_{2}{ }^{\prime}\right)$ is derived also from $\left(11_{2}{ }^{\prime}\right)$ and so contains a form of minimum greater than 1 ; we list such forms also for the genera with the residues $\left(13_{1}{ }^{\prime}\right),\left(13_{1}{ }^{\prime \prime}\right)$, and $\left(13_{2}{ }^{\prime \prime}\right)$ :

$$
\begin{gathered}
4 x_{0}{ }^{2}+4 x_{0} x_{1}+5 x_{1}{ }^{2}+4 x_{2}{ }^{2}+4 x_{3}{ }^{2}, \\
5\left(x_{0}+2 x_{1} / 5+x_{2} / 5\right)^{2}+4\left(4 x_{1}{ }^{2}+4 x_{1} x_{2}+6 x_{2}{ }^{2}+5 x_{3}{ }^{2}\right) / 5 \\
5\left(x_{0}+x_{1} / 5-2 x_{3} / 5\right)^{2}+\left(29 x_{1}{ }^{2}+20 x_{2}{ }^{2}+16 x_{3}{ }^{2}-20 x_{1} x_{2}-16 x_{1} x_{3}\right) / 5
\end{gathered}
$$


In the genus determined by $\left(13_{3}{ }^{\prime}\right) \bmod 2^{r}$ we find the form

$$
F_{04}=x_{0}^{2}+8\left(x_{1}^{2}+x_{2}^{2}+x_{3}^{2}+x_{1} x_{2}+x_{1} x_{3}+x_{2} x_{3}\right) \text {. }
$$

Curiously enough, this case is missing in Smith [13, vol. II, pp. 669-670] (here $I_{1}=4, I_{2}=1, I_{3}=4$, and $\theta_{1}, \theta_{2}, \theta_{3} \equiv 1,0,1, \bmod 2$, so that our case would have come under Smith's $E(b))$. However, if $a$ is the minimum of a form in the genus of $F_{64}, a^{4} \leqq 1024, a \leqq 5$. But $a=2,3$, and 5 are not represented by forms in this genus. Further, 4 is not represented primitively. For if $F_{64} \equiv 4$ $(\bmod 2 r), x_{0} \equiv 2(\bmod 4), x_{0}{ }^{2} \equiv 4(\bmod 32), x_{1}{ }^{2}+x_{2}{ }^{2}+x_{3}{ }^{2}+x_{1} x_{2}+x_{1} x_{3}+x_{2} x_{3} \equiv 0$ ( $\bmod 4)$, every $x_{\alpha}$ is even. Hence all forms in the genus represent 1 , and by Theorem 9, all such forms are norm-forms. But the genus of the ternary form $f=3 x_{1}^{2}+3 x_{2}^{2}+3 x_{3}^{2}-2 x_{1} x_{2}-2 x_{1} x_{3}-2 x_{2} x_{3}$ contains only one class. The same follows for $F_{64}$.

VI. Forms $G$ of determinant 1024 . We have only to apply transformations of determinant 2 to $\left(13_{3}{ }^{\prime}\right)$. The resulting form-residues are

$$
x_{0}{ }^{2}+192 x_{3}{ }^{2}+8\left(x_{1}{ }^{2}+x_{1} x_{2}+x_{2}{ }^{2}\right), \quad x_{0}{ }^{2}+48 x_{3}{ }^{2}+8 x_{1}{ }^{2}+24 x_{2}{ }^{2}, \quad \bmod 2^{r} \text {. }
$$

The latter form is derivable from $(1,3,8,24)$ in $\left(11^{\prime}\right)$, and so its genus contains a class of minimum greater than 1. To get a form of minimum $a$ in the genus of the first form, note that $a^{4} \leqq 4 \cdot 1024, a=1$, 4 , or 8 . Trying $a=4$ we consider $4 x_{0}^{2}+4 x_{0} x_{3}+k x_{3}{ }^{2}+\cdots$, and try to satisfy $4 k-4=192$. Hence we take $k=49$, and consider $4\left(x_{0}+x_{3} / 2\right)^{2}+\phi\left(x_{1}, x_{2}, x_{3}\right), \phi \equiv 48 x_{3}{ }^{2}+\cdots$ $\sim 48 x_{3}{ }^{2}+8\left(x_{1}{ }^{2}+x_{1} x_{2}+x_{2}{ }^{2}\right) \bmod 2^{r}$. Here det $\phi$ must be 256 , and adj $\phi=16 \psi$, where $\psi \sim 3 x_{3}{ }^{2}+24\left(x_{1}{ }^{2}-x_{1} x_{2}+x_{2}{ }^{2}\right) \bmod 2^{r}$, det $\psi=16$, and adj $\psi=\phi$. A possible form $\psi$ is easily found to be $3 x_{1}^{2}+3 x_{2}^{2}+3 x_{3}{ }^{2}-2 x_{1} x_{2}-2 x_{1} x_{3}-2 x_{2} x_{3}$, and we construct

$$
\begin{aligned}
4\left(x_{0}+x_{1} / 2+x_{2} / 2+\right. & \left.x_{3} / 2\right)^{2}+8 x_{1}{ }^{2}+8 x_{2}{ }^{2}+8 x_{3}{ }^{2}+8 x_{1} x_{2}+8 x_{1} x_{3}+8 x_{2} x_{3} \\
= & 4 x_{0}{ }^{2}+9 x_{1}{ }^{2}+9 x_{2}{ }^{2}+9 x_{3}{ }^{2}+4 x_{0} x_{1}+4 x_{0} x_{2}+4 x_{0} x_{3} \\
& +10 x_{1} x_{2}+10 x_{1} x_{3}+10 x_{2} x_{3},
\end{aligned}
$$

which is in the desired genus, since

$$
\begin{aligned}
9\left(x_{1}+5 x_{2} / 9+2 x_{0} / 9+5 x_{3} / 9\right)^{2}+8\left(7 x_{2}{ }^{2}+\right. & \left.5 x_{2} x_{3}+7 x_{3}{ }^{2}+4 x_{0}{ }^{2}+2 x_{0} x_{2}+2 x_{0} x_{3}\right) / 9 \\
& \sim x_{1}{ }^{2}+8\left(x_{2}{ }^{2}+x_{2} x_{3}+x_{3}{ }^{2}+\kappa x_{0}{ }^{2}\right), \bmod 2 \text { r. }
\end{aligned}
$$

where by the determinant, $\kappa$ can only be 24 .

All further genera derived from these must contain a class of minimum greater than 1.

Consider now the genera containing norm-form classes only, which can be derived from $F_{3}=x_{0}{ }^{2}+x_{0} x_{3}+x_{3}{ }^{2}+x_{1}{ }^{2}+x_{1} x_{2}+x_{2}{ }^{2}$ by transformations of determinant 2 . Now $c_{3}=-1, c_{2}=1$. Hence in (2) and (3), either $j=1$ and $\lambda$ contains an even power of 2 , or $j=0$. The residue $F_{3} \sim x_{0}{ }^{2}+3 x_{3}{ }^{2}+x_{1}{ }^{2}+3 x_{2}{ }^{2} \bmod 3^{r}$ is not changed by transformations of determinant prime to 3 . 
I. Forms of determinant $9 / 4$. The preceding conditions allow only one genus, that with the form-residue $x_{0}^{2}+x_{0} x_{3}+2 x_{1} x_{2} \bmod 2^{r}$. This genus has only one class, represented by

$F_{0}=\left(x_{0}+x_{1} / 2+x_{2} / 2\right)^{2}+\left(x_{3}+x_{1} / 2+x_{2} / 2\right)^{2}+3\left(x_{1}{ }^{2}+x_{2}{ }^{2}\right) / 2$

II. Forms of determinant 9 . There are three genera, each of one class, containing the respective forms

$$
\begin{aligned}
F_{12}= & x_{0}{ }^{2}+3 x_{1}{ }^{2}+3 x_{2}{ }^{2}+x_{3}{ }^{2}, \\
F_{12}{ }^{\prime}= & x_{0}{ }^{2}+x_{0} x_{3}+x_{3}{ }^{2}+4\left(x_{1}{ }^{2}+x_{1} x_{2}+x_{2}{ }^{2}\right), \\
F_{12}{ }^{\prime \prime}= & \left(x_{0}+x_{1} / 2+x_{2} / 2+x_{3} / 2\right)^{2} \\
& \quad+\left(15 x_{1}{ }^{2}+7 x_{2}{ }^{2}+7 x_{3}{ }^{2}-6 x_{1} x_{2}-6 x_{1} x_{3}-2 x_{2} x_{3}\right) / 4 .
\end{aligned}
$$

Here $F_{12}{ }^{\prime \prime}$ has the form-residue $x_{0}{ }^{2}+x_{0} x_{3}+4 x_{1} x_{2} \bmod 2^{r}$, and is derived from $F_{6} ; F_{12}$ is derived by the transformation $x_{0}=y_{0}-y_{2}, x_{1}=y_{1}+y_{2}, x_{2}=-y_{2}+y_{3}$, $x_{3}=-y_{2}+y_{3}$, of determinant 2 , from $F_{6}$. But $F_{12}{ }^{\prime}$ is derived by a transformation of determinant 4 from $F_{3}$.

III. Forms of determinant 36. The form

$$
F_{24}=x_{0}{ }^{2}+3 x_{3}{ }^{2}+4\left(x_{1}^{2}+x_{1} x_{2}+x_{2}{ }^{2}\right)
$$

is in a genus of one class, and can be derived from any of $F_{12}, F_{12}{ }^{\prime}, F_{12}{ }^{\prime \prime}$. The genera with the form-residues $x_{0}{ }^{2}+6 x_{1}{ }^{2}+6 x_{2}{ }^{2}+x_{3}{ }^{2}$ and $x_{0}{ }^{2}+x_{0} x_{3}+8 x_{1} x_{2}$, mod $2^{r}$, contain the following forms of minimum 2 :

$$
\begin{aligned}
2 x_{0}{ }^{2}+2 x_{1}{ }^{2}+ & 3 x_{2}{ }^{2}+3 x_{3}{ }^{2}, \quad 2\left(x_{0}+x_{1} / 4+x_{2} / 2+x_{3} / 2\right)^{2} \\
& +\left(15 x_{1}{ }^{2}+28 x_{2}{ }^{2}+28 x_{3}{ }^{2}-12 x_{1} x_{2}-12 x_{1} x_{3}-8 x_{2} x_{3}\right) / 8
\end{aligned}
$$

IV. Forms of determinant 144 . We must now apply transformations of determinant 2 to (18). We thus get the genus of one class containing

$$
F_{48}=x_{0}^{2}+12 x_{3}^{2}+4\left(x_{1}^{2}+x_{1} x_{2}+x_{2}^{2}\right) ;
$$

and the genera with the form-residues

$$
x_{0}^{2}+3 x_{1}^{2}+4 x_{2}^{2}+12 x_{3}^{2}, \quad x_{0}^{2}-x_{3}^{2}+8 x_{1} x_{2},
$$

$\bmod 2 r$

The latter is derivable from $\left(18_{2}{ }^{\prime}\right)$; the former genus contains the form

$$
5 x_{0}{ }^{2}+4 x_{1}{ }^{2}+4 x_{2}{ }^{2}+4 x_{3}{ }^{2}+2 x_{0} x_{1}-4 x_{0} x_{2}+4 x_{0} x_{3}+4 x_{1} x_{3}
$$

of minimum 4.

V. Forms of determinant $144 \cdot 2^{2 s}$. The only genus derived from (19) by suitable transformations of determinant 2 is that containing $x_{0}{ }^{2}+12 x_{1}{ }^{2}+12 x_{2}{ }^{2}$ $+4 x_{3}{ }^{2}$, and being derived also from $\left(19_{1}^{\prime}\right)$, it contains a form of minimum greater than 1 .

Proceeding next from $F_{5}$, whence $c_{2}=1$ and $c_{5}=-1$, we have $F_{5} \sim x_{1}{ }^{2}+3 x_{1}{ }^{2}$ $+5 x_{2}{ }^{2}+15 x_{3}{ }^{2} \bmod 5^{r}$. 
I. Forms of determinant $25 / 4$. The only residue $\bmod 2^{r}$ is $x_{0}{ }^{2}+x_{0} x_{3}+2 x_{1} x_{2}$, and this gives a genus of one class, containing

$$
F_{10}=\left(x_{0}+x_{2} / 2+x_{3} / 2\right)^{2}+\left(12 x_{1}{ }^{2}+7 x_{2}{ }^{2}+7 x_{3}{ }^{2}-4 x_{1} x_{2}-4 x_{1} x_{3}-6 x_{2} x_{3}\right) / 4 .
$$

II. Forms of determinant 25. The possible genera have the form-residues

$$
\begin{gathered}
x_{0}{ }^{2}+x_{1}{ }^{2}-x_{2}{ }^{2}-x_{3}{ }^{2}, x_{0}{ }^{2}+x_{0} x_{3}+x_{3}{ }^{2}+4\left(x_{1}{ }^{2}+x_{1} x_{2}+x_{2}{ }^{2}\right), \\
x_{0}{ }^{2}+x_{0} x_{3}+4 x_{1} x_{2}, \bmod 2^{r} .
\end{gathered}
$$

The first contains the form

$$
F_{20}=x_{0}{ }^{2}+5 x_{1}{ }^{2}+3 x_{2}{ }^{2}+2 x_{2} x_{3}+2 x_{3}{ }^{2}
$$

in a genus of one class; and the other two contain the following forms of minimum greater than 1 :

$$
\begin{aligned}
& 3 x_{0}{ }^{2}+3 x_{1}{ }^{2}+3 x_{2}{ }^{2}+3 x_{3}{ }^{2}+2 x_{0} x_{1}+3 x_{0} x_{2}+3 x_{0} x_{3}-x_{1} x_{2}-x_{1} x_{3}+x_{2} x_{3}, \\
& 3 x_{0}{ }^{2}+3 x_{1}{ }^{2}+2 x_{2}{ }^{2}+2 x_{3}{ }^{2}+2 x_{0} x_{1}-x_{0} x_{2}-x_{0} x_{3}-x_{1} x_{2}-x_{1} x_{3}-x_{2} x_{3} .
\end{aligned}
$$

III. Forms of determinant 100. Suitable transformations on $x_{0}{ }^{2}+x_{1}{ }^{2}-x_{2}{ }^{2}$ $-x_{3}^{2} \bmod 2^{r}$ are $x_{1} \rightarrow 2 x_{1}+x_{2}$; and $x_{2} \rightarrow 2 x_{2}+x_{3}$; the resulting form-residues are $x_{0}{ }^{2}+x_{1}{ }^{2}-4 x_{2}{ }^{2}-4 x_{2} x_{3}-2 x_{3}{ }^{2} \sim x_{0}{ }^{2}+x_{1}{ }^{2}-2 x_{2}{ }^{2}-2 x_{3}{ }^{2}$, and $x_{0}{ }^{2}+4 x_{1}{ }^{2}+4 x_{1} x_{2}-x_{3}{ }^{2}$ $\sim x_{0}^{2}+4 x_{1} x_{2}-x_{3}{ }^{2}$. The following forms in these genera have minimum greater than 1:

$$
\begin{gathered}
3 x_{0}{ }^{2}+2 x_{0} x_{3}+2 x_{3}{ }^{2}+6 x_{1}{ }^{2}+4 x_{1} x_{2}+4 x_{2}{ }^{2}, \\
5 x_{1}{ }^{2}+3 x_{0}{ }^{2}+3 x_{2}{ }^{2}+3 x_{3}{ }^{2}+2 x_{0} x_{2}+2 x_{0} x_{3}+2 x_{2} x_{3} .
\end{gathered}
$$

Proceeding similarly from $F_{7}$, the derived genus of determinant $49 / 4$ with the residue $x_{0}^{2}+x_{0} x_{3}+2 x_{1} x_{2}$ mod $2^{r}$ contains the form

$$
2 x_{0}^{2}+2 x_{1}^{2}+2 x_{2}^{2}+2 x_{3}^{2}+x_{0} x_{2}-x_{0} x_{3}+x_{1} x_{2}+x_{1} x_{3}
$$

of minimum 2. This disposes, for determinant 49 , of the residue $x_{0}{ }^{2}+2 x_{0} x_{8}$ $+2 x_{1} x_{2} \sim\left(x_{0}+x_{3}\right)^{2}-x_{3}{ }^{2}+2 x_{1} x_{2} \sim x_{0}{ }^{2}+x_{1}{ }^{2}-x_{2}{ }^{2}-x_{3}{ }^{2}$ mod $2^{r}$; or else note the form $3 x_{0}{ }^{2}+3 x_{1}{ }^{2}+3 x_{2}{ }^{2}+3 x_{3}{ }^{2}+2 x_{0} x_{2}-2 x_{0} x_{3}+2 x_{1} x_{2}+2 x_{1} x_{3}$ of minimum 3 . The residue $\left(21_{3}{ }^{\prime}\right)$ also is eliminated. But $\left(21_{2}{ }^{\prime}\right)$ gives the form

$$
F_{28}=\left(x_{0}+x_{2} / 2+x_{3} / 2\right)^{2}+\left(32 x_{1}{ }^{2}+11 x_{2}{ }^{2}+11 x_{3}{ }^{2}-8 x_{1} x_{2}-8 x_{1} x_{3}-6 x_{2} x_{3}\right) / 4
$$

in a genus of one class.

All genera of norm-forms derived from $F_{13}$ by transformations of determinant $2^{8}$ contain classes of minimum greater than 1 . For, the residue $x_{0}{ }^{2}+x_{0} x_{3}$ $+2 x_{1} x_{2} \bmod 2^{r}$ and determinant $169 / 4$ belong to the form

$$
2\left(x_{0}+x_{4} / 4-x_{2} / 2-x_{3} / 4\right)^{2}+\left(31 x_{1}{ }^{2}+28 x_{2}{ }^{2}+15 x_{3}{ }^{2}-12 x_{1} x_{2}-14 x_{1} x_{3}-4 x_{2} x_{3}\right) / 8 \text {. }
$$

The residues $\left(21_{1}{ }^{\prime}\right)$ and $\left(21_{3}{ }^{\prime}\right)$ are eliminated as before; but $\left(21_{2}{ }^{\prime}\right)$ yields a genus of determinant 169 containing the form of minimum 3 ,

$$
3\left(x_{0}+x_{1} / 6+x_{2} / 6-x_{3} / 3\right)^{2}+\left(59 x_{1}{ }^{2}+59 x_{2}{ }^{2}+32 x_{3}{ }^{2}-38 x_{1} x_{2}-8 x_{1} x_{3}-8 x_{2} x_{3}\right) / 12 \text {. }
$$


14. The norm-forms permitting factorization with $m$ subject only to $\$ 5(1)$. Every norm-form $G$ is derivable from a norm-form $F$ in which adj $f$ is fundamental. If $F$ is not equivalent to one of the five forms $F_{d}$, the genus of $F$ will contain a class of minimum greater than 1 , and the same will hold for $G$. It is therefore sufficient to consider only forms derived from the $F_{d}$.

Let $F_{d}=\left(x_{0}+2^{-1} \sum \epsilon_{\alpha} x_{\alpha}\right)^{2}+\sum A_{\alpha \beta} x_{\alpha} x_{\beta}$. If $G$ is derived from $F_{d}$ by the application of an integral transformation $T$ of determinant $\tau$ to the $x_{\alpha}$, we have

$$
\begin{aligned}
G=\left(r_{0}+2^{-1} \sum \epsilon_{\alpha}{ }^{*} r_{\alpha}\right)^{2}+\sum B_{\alpha \beta} r_{\alpha} r_{\beta}, \quad B=T^{\prime} A T, \quad \xi=T \rho \\
\text { where } \rho^{\prime}=\left(r_{1}, r_{2}, r_{3}\right), \quad x_{0}+2^{-1} \sum \epsilon_{\alpha} x_{\alpha}=r_{0}+2^{-1} \sum \epsilon_{\alpha}{ }^{*} r_{\alpha} .
\end{aligned}
$$

If we should apply a full transformation of order 4 to $F_{d}$ we would have a more difficult discussion of suitability of transformations and of genera. However, after narrowing the problem a little we shall use such full transformations.

We saw in $\$ 11$ that, for certain integral matrices $S$ of determinant $\sigma$, where $\sigma=4$ if $d=2,5$, and 13 , and $\sigma=2$ if $d=3$ and 7 , the transformation

$$
\eta=S \xi, \quad x_{0}+2^{-1} \sum \epsilon_{\alpha} x_{\alpha}=y_{0} / 2,
$$

replaces $F_{d}$ by the form $H_{d}$, where

$$
4 e^{2} H_{d}=e^{2} y_{0}^{2}+e^{2} d_{1} y_{1}^{2}+e d_{1} y_{2}^{2}+e y_{3}^{2},
$$

$d_{1}=$ odd part of $d, e=1$ for $d=2,3,7, e=2$ for $d=5,13$. Also, (2) replaces the system $\Sigma_{F}$ of integral quaternions associated with $F_{d}$ by the arithmetically equivalent system $\Sigma_{d}(d=2,3,7,5,13)$.

As in Lemma 14, we can for the given $S$ and $T$ choose integral matrices $S_{1}$ and $T_{1}$ of determinants $\sigma$ and $\tau$, such that $S T=T_{1} S_{1}$. Hence $T=S^{-1} T_{1} S_{1}$ and replaces $A$ by $B$. Let $\tau$ be odd. Consider, after (2), the transformations

$$
\begin{aligned}
\eta & =T_{1} \zeta, & y_{0} / 2 & =z_{0} / 2, \\
\zeta & =S_{1} \rho, & z_{0} / 2 & =r_{0}+2^{-1} \sum \epsilon_{\alpha}{ }^{*} r_{\alpha} .
\end{aligned}
$$

On applying (4) to $\Sigma_{d}$ we obtain a subsystem $\Sigma_{d}$ 'by imposing the restriction on the $y_{\alpha}$ that the $z_{\alpha}$ be integers, that is, that $T_{1}^{-1} \eta$ be integral $\bmod \tau$; or if we prefer to carry through the substitution, we obtain a system $\Sigma_{d}{ }^{\prime \prime}$ arithmetically equivalent to $\Sigma_{d}{ }^{\prime}$, with elements expressible as $z_{0} / 2+z_{1} i_{1}{ }^{\prime \prime} / 2+\ldots$ where now the $z_{i}$ are unrestricted $\bmod \tau$ but still satisfy relations mod 2 or 4 due to those on the $y_{i}$ in $\Sigma_{d}$. As an algebra, the system $\Sigma_{d}^{\prime \prime}$ must be the same as that obtained by application of (5) from the system of integral quaternions $\Sigma_{G}$. Further, the conditions of integrality are the same, it being assumed that $\tau$ is odd: for, the condition that $S_{1}^{-1} \zeta(=\rho)$ be integral mod $\sigma$ is the same as the condition that $S^{-1} T_{1} \zeta(=\xi)$ be integral mod $\sigma$, since $S^{-1} T_{1}=T S_{1}^{-1}$ and $T^{-1}$ is integral $\bmod \sigma$.

Hence if we prove by one instance that factorization is not possible in $\Sigma_{d}$, 
the same will follow for $\Sigma_{G}$, whence the genus of $G$ will contain a class of minimum greater than 1 .

Since $T_{1} S_{1}(=S T)$ replaces $\phi=e^{2} d_{1} y_{1}{ }^{2}+e d_{1} y_{2}{ }^{2}+e y_{3}{ }^{2}$ by $4 e^{2} \sum B_{\alpha \beta} r_{\alpha} r_{\beta}$, clearly $T_{1}$ is a suitable tranformation for $\phi$.

We shall now apply to the $y_{\alpha}$ the suitable transformations $T_{1}$ of determinant $p$ or $p^{2}$, where, to begin with, $p \nmid 2 d$. The genus of the form $G$ so obtained must represent 2 . For, $F_{d}$ represents 2 , and since $|T|\left(=\left|T_{1}\right|\right)$ is prime to $2 d$, we can solve the congruences $G \equiv 2\left(\bmod 2^{r}\right.$ and $\left.\bmod d^{r}\right)$. To prove that we can solve $G \equiv 2\left(\bmod p^{r}\right)$, where $p$ is now the only other prime in the determinant of $G$, we note that $G$ is carried by (5) (of determinant prime to $p$ ) into $z_{0}^{2} / 4+\psi / 4 e^{2}$, where $\psi$ is as follows (cf. paragraph containing (11) in \$12):

$$
\begin{array}{ll} 
& e^{2} d_{1}\left(p z_{1}+\lambda z_{2}+\mu z_{3}\right)^{2}+e d_{1} z_{1}{ }^{2}+e z_{3}{ }^{2}, \\
& e^{2} d_{1} z_{1}{ }^{2}+e d_{1}\left(p z_{2}+\nu z_{3}\right)^{2}+e z_{3}{ }^{2}, \\
\text { (6) } & e^{2} d_{1} z_{1}{ }^{2}+e d_{1} p^{2} z_{2}{ }^{2}+e p^{2} z_{3}{ }^{2}, \\
& e^{2} d_{1}\left(p z_{1}+\lambda^{\prime} z_{2}\right)^{2}+e d_{1} z_{2}{ }^{2}+e p^{2} z_{3}{ }^{2}, \\
& e^{2} d_{1}\left(p z_{1}+\mu^{\prime} z_{3}\right)^{2}+e d_{1}\left(p z_{2}+\nu^{\prime} z_{3}\right)^{2}+e z_{3}{ }^{2},
\end{array}
$$

$$
\begin{aligned}
& \text { if } p \mid 1+e \lambda^{2}+e d_{1} \mu^{2} \text {; } \\
& \text { if } p \mid e+e d_{1} \nu^{2} \text {; } \\
& \text { if }\left(-d_{1} \mid p\right)=-1 \text {; } \\
& \text { if } p \mid 1+e \lambda^{\prime 2} \text {; }
\end{aligned}
$$

if $p \nmid e+e^{2} d_{1} \mu^{\prime 2}+e d_{1} \nu^{\prime 2}$.

In each case $\phi$ has a coefficient $m z_{\alpha}{ }^{2}$ where $p \nmid m$, and the solvability of $G \equiv 2$ follows from that of $z_{0}^{2}+m z_{\alpha}^{2} \equiv 2\left(\bmod p^{r}\right)$.

The quaternions of norm 2 in the various $\Sigma_{d}$ are easily found by solving

$$
e y_{0}^{2}+e d_{1} y_{1}^{2}+d_{1} y_{2}^{2}+y_{3}^{2}=8 e,
$$

subject to the restrictions on the $y_{i}$ in $(1),(3),(6),(7)$ of $\$ 11$, and are as follows :

$$
\begin{aligned}
& d=2: \pm\left(1 \pm i_{1}\right), \pm\left(1 \pm i_{2}\right), \pm\left(1 \pm i_{3}\right), \pm\left(i_{2} \pm i_{3}\right), \pm\left(i_{3} \pm i_{1}\right), \pm\left(i_{1} \pm i_{2}\right) ; \\
& d=3: \pm\left(1 \pm i_{3}\right), \pm\left(2 \pm i_{2} \pm i_{3}\right) / 2, \pm\left(1 \pm i_{1} \pm 2 i_{3}\right) / 2, \pm\left(1 \pm i_{1} \pm i_{2} \pm i_{3}\right) ; \\
& d=7: \pm\left(1 \pm i_{3}\right), \pm\left(1 \pm i_{1}\right) / 2, \pm\left(i_{2} \pm i_{3}\right) / 2 ; \\
& d=5: \pm\left( \pm 2 i_{1}+i_{2}+i_{3}\right) / 4, \pm\left( \pm 2+i_{2}+3 i_{3}\right) / 4, \pm\left(1 \pm i_{1} \pm i_{3}\right) / 2, \pm i_{3} ; \\
& d=13: \pm\left( \pm 2+i_{2}-i_{3}\right) / 4, \pm i_{3} .
\end{aligned}
$$

At the same time, we record the quaternions of norm 1:

$$
\begin{aligned}
& d=2: \quad \pm 1, \pm i_{1}, \pm i_{2}, \pm i_{3}, \pm\left(1 \pm i_{1} \pm i_{2} \pm i_{3}\right) / 2 \text {; } \\
& d=3: \pm 1, \pm i_{3}, \pm\left(1 \pm i_{1}\right) / 2, \pm\left(i_{2} \pm i_{3}\right) / 2 ; \quad d=7: \pm 1, \pm i_{3} ; \\
& d=5: \pm\left( \pm 2+i_{2}-i_{3}\right) / 4, \pm 1 ; \quad d=13: \pm 1 \text {. }
\end{aligned}
$$

Suppose that $T_{1}$ is $P_{\lambda \mu}$ of $\S 12$. Then in $\Sigma_{d}{ }^{\prime}, y_{1} \equiv \lambda y_{2}+\mu y_{3} \bmod p$, and this is satisfied by quaternions of norm 2 as follows: by $1 \pm i_{1}$, never; $1 \pm i_{2}$ only if $\lambda \equiv 0 ; 1 \pm i_{3}$ and $i_{3}$ if $\mu \equiv 0 ; i_{2} \pm i_{3}$ and $2 \pm i_{2} \pm i_{3}$ if $\lambda \equiv \pm \mu ; i_{1} \pm i_{3}$ and $1 \pm i_{1} \pm i_{3}$ if $\mu \equiv \pm 1 ; i_{1} \pm i_{2}$ if $\lambda \equiv \pm 1 ; 1 \pm i_{1} \pm 2 i_{3}$ if $2 \mu \equiv \pm 1 ; 1 \pm i_{1} \pm i_{2} \pm i_{3}$ if $\pm \lambda \pm \mu \equiv \pm 1$; 
$\pm 2 i_{1}+i_{2}+i_{3}$ if $\lambda+\mu \equiv \pm 1 ; \pm 2+i_{2}+3 i_{3}$ if $\lambda \equiv-3 \mu ; \pm 2+i_{2}-i_{3}$ if $\lambda \equiv \mu, \bmod p$.

Now the number of solutions $\lambda, \mu \bmod p$ of $1+e \lambda^{2}+e d_{1} \mu^{2} \equiv 0$ is $p-\left(-d_{1} \mid p\right)$. Of these, the number with $\lambda \equiv 0$ is $1+\left(-e d_{1} \mid p\right)$; the number with $\mu \equiv 0$ is $1+(-e \mid p)$; the number with $\lambda \equiv \pm \mu$ is $2[1+(-2 \mid p)]$ if $d=2$ or 7 , $2[1+(-1 \mid p)]$ if $d=3$; the number with $\lambda \equiv-3 \mu$ is 0 if $d=5$ and $p=7$, but $1+(-7 \mid p)$ if $d=5$ and $p \neq 7$; the number with $\lambda \equiv \mu$ is 0 if $d=13$ and $p=7$, $1+(-7 \mid p)$ if $d=13$ and $p \neq 7$; the number with $\mu \equiv \pm 1$ is $2[1+(-2 \mid p)]$ if $d=2,2[1+(-22 \mid p)]$ if $d=5$; the number with $\lambda \equiv \pm 1$ is $2[1+(-2 \mid p)]$ if $d=2$; the number with $2 \mu \equiv \pm 1$ is $2[1+(-7 \mid p)]$ if $d=3$; the number with $\pm \lambda \pm \mu \equiv 1$ is $4[1+(-7 \mid p)]$ if $d=3$; if $d=5$, the number with $\lambda+\mu \equiv \pm 1$ is 2 if $p=3,2[1+(-2 \mid p)]$ if $p>5$. Counting these as they come, not worrying about duplicates, we find at most $n_{d}$ solutions $(\lambda, \mu)$ for which a quaternion of norm 2 may belong to $\Sigma_{d}{ }^{\prime}$, where

$$
n_{d}=\left\{\begin{array}{l}
8+2(-1 \mid p)+6(-2 \mid p) \\
9+3(-1 \mid p)+6(-7 \mid p) \\
3+(-1 \mid p)+2(-2 \mid p) \\
6+3(-2 \mid p)+(-7 \mid p)+2(-22 \mid p) \\
2+(-2 \mid p)+(-7 \mid p)
\end{array}\right.
$$

if $d=2$, if $d=3$, if $d=7$, if $d=5, p>7$, if $d=13, p \neq 7$;

while $n_{d}=0$ if $d=5$ or 13 and $p=7$. Hence there exist solutions of $1+e \lambda^{2}+e d_{1} \mu^{2}$ $\equiv 0 \bmod p$, for which $\Sigma_{d}$ contains no quaternions of norm 2 , in the following cases:

$$
\begin{aligned}
& d=2, \text { if } p=7, p=13, \text { or } p \geqq 19 ; \quad d=3, \text { if } p \geqq 13 ; \\
& d=7, \text { if } p \geqq 3 ; \quad d=5, \text { if } p \geqq 7 ; \quad d=13, \text { if } p \geqq 3 .
\end{aligned}
$$

If $T_{1}$ is $P_{\nu}$ of $\S 12$, which requires that $p \mid 1+d_{1} \nu^{2}$ and $y_{2} \equiv \nu y_{3} \bmod p$, we find a value of $\nu$ for which $\Sigma_{d}$ contains no quaternion of norm 2 in the additional case $d=5$ and $p=3$; that is, if we take $\nu=-1$, no quaternion of norm 2 satisfies $y_{2} \equiv-y_{3} \bmod 3$.

Now only one genus of norm-forms $G$ is obtained from $F_{d}$ by all the suitable transformations of a given determinant $p$ not dividing $2 d$. For, the formresidue $\bmod p_{1}{ }^{r}$ of $G$ is determined by that of $F_{d}$ if $p_{1} \neq p$; and if $p_{1}=p$, of the two apparent possibilities $(1, n, p, n p)$, where $n$ may be a quadratic residue or non-residue, only one is possible, since $c_{p}=(-n \mid p)$, and $c_{p}$ is invariant under all rational transformations. Here, $c_{p}$ is +1 , and we can take $n=-1$.

The preceding paragraph holds also if the transformations of determinant $p$ are of order 4 , the forms so obtained being necessarily in the genus of $G$, though not necessarily norm-forms. If $T_{2}$ is of determinant $p$ and order 4 , and $S_{2}$ denotes the integral transformation of order 4 and determinant $2 \sigma$ which replaces $H_{d}$ by $F_{d}$, then we can choose integral matrices $S_{3}$ and $T_{3}$, of determinants $2 \sigma$ and $p$, such that $T_{2} S_{3}=S_{2} T_{3}$. For if we define $S_{2}{ }^{*}$ by $S_{2}{ }^{*} S_{2}=2 \sigma I$, we 
have $S_{2}{ }^{*} T_{2}=T_{3} S_{4}$, and $2 \sigma T_{2}=S_{2} T_{3} S_{4}$. Hence $2 \sigma S_{4}^{-1}=T_{2}^{-1}\left(S_{2} T_{3}\right)$. The left side is integral $\bmod p$, the right side $\bmod 2$. Hence $2 \sigma S_{4}^{-1}$ is an integral matrix, say $S_{3}$; and $T_{2} S_{3}=S_{2} T_{3}$. Now if $T_{3}$ replaces $F_{d}$ by a form $G_{1}$ with a formresidue $(1,-1, p,-p) \bmod p^{r}$, then $G_{1}$ is in the genus of $G$. Also, the transformation $T_{2} S_{3}$ replaces $H_{d}$ by $G_{1}$.

We thus have four forms: $F_{d}, H_{d}, H_{d}{ }^{\prime}$, and $G_{1}$; where $H_{d}{ }^{\prime}$ is obtained from $H_{d}$ by the transformation $T_{2}$; and hence $G_{1}$ from $H_{d}{ }^{\prime}$ by $S_{3}$. The variables $y_{i}$ in $H_{d}$ are to be subjected to the conditions mod 2 or 4 of the system $\Sigma_{d}$; the variables $z_{i}$ in $H_{d}{ }^{\prime}$ are to be subjected to the conditions mod 2 or 4 such that, under $S_{3}$, the variables of $G_{1}$ are arbitrary integers. If we now prove that $H_{d}{ }^{\prime}$, so conditioned, does not represent 1 , the same holds for $G_{1}$. Since $\left|T_{2}\right|$ is odd, the conditions of integrality on the $z_{i} \bmod 2$ or 4 must be equivalent to the conditions on the $y_{i}$, connected with the $z_{i}$ by $T_{2}$.

Let us therefore apply to $H_{d}$ the transformation

$$
T_{2}: \quad y_{0}=p z_{0}+\lambda_{1} z_{1}+\lambda_{2} z_{2}+\lambda_{3} z_{3}, \quad y_{1}=z_{1}, \quad y_{2}=z_{2}, \quad y_{3}=z_{3} .
$$

LEMMA 17. Let $a_{0} a_{1} a_{2} a_{3}$ be prime to $p, p>2$. Then every third order minor determinant in the matrix of $\psi\left(z_{0}, z_{1}, z_{2}, z_{3}\right)=a_{0}\left(p z_{0}+\sum \lambda_{\alpha} z_{\alpha}\right)^{2}+\sum a_{\alpha} z_{\alpha}{ }^{2}$ is divisible by $p$ if and only if

$$
p \mid a_{1} a_{2} a_{3}+a_{0} a_{2} a_{3} \lambda_{1}{ }^{2}+a_{0} a_{1} a_{3} \lambda_{2}{ }^{2}+a_{0} a_{1} a_{2} \lambda_{3}{ }^{2} .
$$

Hence the form $4 e^{2} H_{d}{ }^{\prime}=e^{2}\left(p z_{0}+\sum \lambda_{\alpha} z_{\alpha}\right)^{2}+e^{2} d_{1} z_{1}{ }^{2}+e d_{1} z_{2}{ }^{2}+e z_{3}{ }^{2}$ has the form-residue $(1, n, p, n p)$ mod $p^{r}$ if and only if

$$
p \mid d_{1}+\lambda_{1}^{2}+e \lambda_{2}^{2}+e d_{1} \lambda_{3}^{2} .
$$

Now 1 will be represented in the form $H_{d}$ ' if and only if the coordinates of one of the units in (9) satisfies $y_{0} \equiv \lambda_{1} y_{1}+\lambda_{2} y_{2}+\lambda_{3} y_{3}$. For $d=2$, this means that either some $\lambda_{\alpha} \equiv 0$ or that $\pm \lambda_{1} \pm \lambda_{2} \pm \lambda_{3}+1 \equiv 0$ for some choice of signs. Now if $p=17$, we have $17 \mid 3^{2}+4^{2}+5^{2}+1$, and $\pm 3 \pm 4 \pm 5+1 \neq 0(\bmod 17)$. Hence $G$ does not represent 1 .

If $d=3,\left(9_{2}\right)$ shows that $H_{d}{ }^{\prime}$ represents 1 if and only if $\lambda_{3} \equiv 0$, or $\lambda_{1} \equiv \pm 1$, or $\lambda_{2} \equiv \pm \lambda_{3}$. Now $11\left|4^{2}+0^{2}+3 \cdot 1^{2}+3,7\right| 2^{2}+2^{2}+3 \cdot 1^{2}+3$, and $5 \mid 2^{2}+0^{2}+3 \cdot 1^{2}$ +3 ; hence if $p$ is 5,7 , or $11, H_{d}{ }^{\prime}$ and $G$ do not represent 1 .

If $d=7$ we need $\lambda_{3} \neq 0$; we have $3 \mid 0^{2}+1^{2}+7 \cdot 1^{2}+7$, eliminating $p=3$.

If $d=13, H_{d}{ }^{\prime}$ cannot represent 1 since $1 \neq 0$.

The transformations (11) of $\$ 12$, of determinant $p^{2}$, lead to two genera, $\Gamma_{1}$ and $\Gamma_{2}$, containing the respective form-residues

$$
\left(1, n, p^{2}, n p^{2}\right) \bmod p^{r},
$$

where $n$ may be either a quadratic residue or non-residue mod $p$. Other apparent possibilities, such as $\left(1, p, n p, n p^{2}\right)$, are excluded by the fact that $\phi$ is primitive in (6), and are indeed derived from the genera obtained previously by transformations of determinant $p$. The genera $\Gamma_{1}$ and $\Gamma_{2}$ are distinguish- 
able by the coefficient $n=e+e^{2} d_{1} \mu^{\prime 2}+e d_{1} \nu^{\prime 2}$ of $z_{3}^{2}$. We seek to determine values of $\mu^{\prime}$ and $\nu^{\prime}$ which make $(n \mid p)$ either +1 or -1 , and such that the conditions

$$
y_{1} \equiv \mu^{\prime} y_{3}, \quad y_{2} \equiv \nu^{\prime} y_{3}(\bmod p)
$$

eliminate all quaternions of norm 2. This is rather easy, since $\mu^{\prime}$ and $\nu^{\prime}$ now satisfy only the incongruence $e+e^{2} d_{1} \mu^{\prime 2}+e d_{1} \nu^{\prime 2} \not \equiv 0(\bmod p)$.

Referring again to (8) we see that (15) fails to hold, for all the quaternions of norm 2, unless: (a) $d=2,\left(\mu^{\prime}, \nu^{\prime}\right) \equiv(0,0),(0, \pm 1)$, or $( \pm 1,0)$; (b) $d=3$, $\left(\mu^{\prime}, \nu^{\prime}\right) \equiv(0,0),(0, \pm 1),( \pm 1 / 2,0)$, or $( \pm 1, \pm 1) ;(\mathrm{c}) d=7,\left(\mu^{\prime}, \nu^{\prime}\right) \equiv(0,0)$, or $(0, \pm 1) ;(\mathrm{d}) d=5,\left(\mu^{\prime}, \nu^{\prime}\right) \equiv( \pm 1,1),( \pm 1,0),(0,0)$, or $\left(3 \mu^{\prime}, 3 \nu^{\prime}\right) \equiv(0,1)$; (e) $d=13,\left(\mu^{\prime}, \nu^{\prime}\right) \equiv(0,0)$ or $(0,-1) \bmod p$. These cases imply, if $d=2$ that $n \equiv 1$ or 2 ; if $d=3$ that $n \equiv 1,4,7 / 4,7$; if $d=7, n \equiv 1,8$; if $d=5, n \equiv 32,28 / 9$, 22 , or 2 ; if $d=13$, that $n \equiv 28$ or $2, \bmod p$. Hence $n$ can certainly be made either a quadratic residue or non-residue, if

$$
d=2, p \geqq 7 ; d=3, p \geqq 7 ; d=7, p \geqq 5 ; d=5, p \geqq 11 ; d=13, p \geqq 7 .
$$

There can also be eliminated the case $d=2, p=5$, since $-\left(1+1^{2}+1^{2} \mid 5\right)=1$ $=\left(1+3 \cdot 1^{2}+3 \cdot 2^{2} \mid 5\right)$; and the case $d=7, p=3,(n \mid p)=-1$, since $\left(1+7 \cdot 1^{2}\right.$ $\left.+7 \cdot 0^{2} \mid 3\right)=-1$. By using $P_{0}{ }^{\prime}$ of $\$ 12$, and the condition $y_{1} \equiv 0, y_{3} \equiv 0(\bmod p)$ we eliminate the case $d=7, p=3$, and $(n \mid 3)=+1$. Let $d=5$; the case $p=7$ is eliminated by $\left(2+20 \cdot 2^{2}+10 \cdot 1^{2} \mid 7\right)=1=-\left(2+20 \cdot 2^{2}+10 \cdot 0^{2} \mid 7\right)$; and the case $p=3$ by $P_{2}^{\prime}$, since no quaternions of norm 2 satisfy $y_{1} \equiv 2 y_{2}, y_{3} \equiv 0(\bmod 3)$. Let $d=13$; the cases $p=3$ and 5 are eliminated by $\left(2+52 \cdot 0^{2}+26 \cdot 1^{2} \mid 3\right)$ $=1=-\left(2+52 \cdot 1^{2}+26 \cdot 1^{2} \mid 3\right)$, and $\left(2+52 \cdot 1^{2}+26 \cdot 0^{2} \mid 5\right)=1=-\left(2+52 \cdot 1^{2}\right.$ $\left.+26 \cdot 2^{2} \mid 5\right)$.

To sum up, we have now eliminated from consideration all cases in which $\tau$ contains a prime factor $p$ not dividing $2 d$, except that it $d=2$, we have to consider the genus obtained by transformations of determinant 3,5 , or 11 , and the genera obtained by transformations of determinant $3^{2}$. (These genera all contain only one class.) We have still to consider genera derived from these, from the genera of $\$ 13$, and from the $F_{d}$ by transformations of determinant divisible by $d$.

Let us consider next the case where $p=d>2$. If $\tau=p$ and the transformation $P_{p}$ of $\S 12$ is used, the genus obtained has the residue $\left(1, p, e p, e p^{2}\right) \bmod p^{r}$. If $p=3$ or 5 , this genus consists of one class. But if $p=7$ or 13 , we can eliminate the genus as follows. The transformation (12) applied to $H_{d}$ produces a form with the residue $\left(1, p, e p, e p^{2}\right) \bmod p^{r}$ if we take $\lambda_{1}=\lambda_{2}=0$ and choose $\lambda_{3}$ so that $\left(e^{2} \lambda_{3}{ }^{2}+e \mid p\right)=1$. If $p=7$ this can be secured with $\lambda_{3}=1$; and it should be noted in $\left(9_{3}\right)$ that no unit satisfies $y_{0} \equiv y_{3}(\bmod 7)$. If $p=13$, take $\lambda_{3}=3$.

If we use transformation $P_{\mu \nu}{ }^{\prime}$, we get the same genus for all $\mu$ and $\nu$, with the residue $\left(1, e, p^{3}, e p^{3}\right) \bmod p^{r}$. This genus represents 2 . The norm- 
form will not represent 2 unless one of the quaternions in (8) satisfies $y_{1} \equiv \mu y_{3}$, $y_{2} \equiv \nu y_{3}, \bmod p$. For $p=5,7$, or 13 , we need merely take $\mu=-\nu=2$. If $p=3$, this method fails; however we easily find in this genus the following form of minimum 2:

$2\left(x_{0}-x_{1} / 4+x_{2} / 2\right)^{2}+\left(31 x_{1}^{2}+28 x_{2}^{2}+16 x_{3}{ }^{2}+4 x_{1} x_{2}+16 x_{1} x_{3}+8 x_{2} x_{3}\right) / 8$.

Thus if $\tau$ contains an odd prime $p$, either $d=2$ and $p=3,5$, or 11 ; or $d=p=3$ or 5 . We must form combinations of these with one another, and the cases of $\$ 13$.

We shall now complete the discussion of the cases arising from $d=2$.

Since $15\left|1+3^{2}+2^{2}+1^{2}, 33\right| 1+9^{2}+4^{2}+1^{2}, 55 \mid 1+7^{2}+2^{2}+1^{4}$, in accordance with (13), we can discard the cases in which $\tau$ is divisible by 15,33 , or 55 , after noting the following.

The genera obtained from $F_{2}$ with $\tau=3^{2}$ contain the respective forms

(16) $F_{18}=\left(x_{0}+x_{1} / 2+x_{2} / 2\right)^{2}+\left(19 x_{1}{ }^{2}+19 x_{2}{ }^{2}+4 x_{3}{ }^{2}+2 x_{1} x_{2}+4 x_{1} x_{3}+4 x_{2} x_{3}\right) / 4$,

(17) $F_{18}{ }^{\prime}=\left(x_{0}+x_{2} / 2\right)^{2}+\left(20 x_{1}{ }^{2}+11 x_{2}{ }^{2}+8 x_{3}{ }^{2}+4 x_{1} x_{2}+8 x_{1} x_{3}+8 x_{2} x_{3}\right) / 4$,

with the form-residues $(1,1,9,9)$ and $(1,2,9,18)$, mod $3^{r}$. Here $F_{18}$ is equivalent to $\left(y_{0}{ }^{2}+9 y_{1}{ }^{2}+9 y_{2}{ }^{2}+y_{3}{ }^{2}\right) / 4$ with the condition $y_{0} \equiv y_{1} \equiv y_{2} \equiv y_{3} \bmod 2$; and $F_{18}{ }^{\prime}$ is equivalent to $\left(y_{0}^{2}+9 y_{1}{ }^{2}+18 y_{2}{ }^{2}+2 y_{3}{ }^{2}\right) / 4$ where $y_{0}=2 x_{0}+x_{2}, y_{1}=x_{2}$, $y_{2}=x_{1}, y_{3}=x_{1}+x_{2}+2 x_{3}$, so that $y_{0} \equiv y_{1}, y_{3} \equiv y_{1}+y_{2}$, mod 2 . Hence the units are \pm 1 and $\pm i_{3}$, and \pm 1 . Further transformations of determinants 5,7 , and 11 give genera containing classes of minimum not 1 . For we have $5 \mid 9+1^{2}+1^{2}$ $+9 \cdot 1^{2}, 7 \mid 9+2^{2}+0^{2}+9 \cdot 2^{2}$, and $11 \mid 9+0^{2}+2^{2}+9 \cdot 1^{2}$, where $\lambda_{3} \neq 0 \bmod p$ (cf. (13)); and 5,7 , and 11 can divide $18+2 \lambda_{1}{ }^{2}+\lambda_{2}{ }^{2}+9 \lambda_{3}{ }^{2}$, without having $\pm 1 \equiv \lambda_{1} \cdot 0+\lambda_{2} \cdot 0+\lambda_{3} \cdot 0 \bmod p$.

Lemma 18. Let $p$ be an odd prime not dividing $d$. Then the norm-forms $G$ derivable from $F_{d}$ by transformations of determinant a power of $p$ have the following form-residues mod $p^{r}$. Here $n, n^{\prime}, n^{\prime \prime}$ denote integers satisfying $(-n \mid p)=1$, $\left(n^{\prime} \mid p\right)=-1,\left(-n^{\prime \prime} \mid p\right)=-1$ :

$$
\begin{gathered}
(1, n, p, n p) ;\left(1,1, p^{2}, p^{2}\right),\left(1, n^{\prime}, p^{2}, n^{\prime} p^{2}\right) ;\left(1, p, n p, n p^{2}\right) ; \\
\left(1, n, p^{3}, n p^{3}\right) ;\left(1, p^{2}, p^{2}, p^{2}\right) ;\left(1, p, n p^{2}, n p^{3}\right),\left(1, n^{\prime} p, n p^{2}, n^{\prime \prime} p^{3}\right) ;
\end{gathered}
$$

and so on. But if $d=p>2$, then the residues $\bmod p^{r}$ are:

$$
\begin{array}{ll}
\left(1, p,-n^{\prime} p,-n^{\prime} p^{2}\right) ; & \left(1,-n^{\prime}, p^{3},-n^{\prime} p^{3}\right) ; \\
\left(1, p,-n^{\prime} p^{2},-n^{\prime} p^{3}\right), & \left(1, n^{\prime} p,-n^{\prime} p^{2},-p^{3}\right) .
\end{array}
$$

These are easily verified, using the properties that $c_{p}=1$ in (18), -1 in (19); that $G$ represents 1 and has the residue $x_{0}^{2}+\phi\left(x_{1}, x_{2}, x_{3}\right)$, where $\phi$ is the adjoint of an integral form.

Besides the forms (16) and (17), we shall now see that $F_{2}$ gives rise by 
transformations of determinant $p^{8}$ to the following forms in genera of one class: if $p=3$;

$$
\begin{aligned}
F_{6}{ }^{\prime}= & x_{0}{ }^{2}+x_{0} x_{3}+x_{3}{ }^{2}+2\left(x_{1}{ }^{2}+x_{1} x_{2}+x_{2}{ }^{2}\right), \\
F_{18}{ }^{\prime \prime}= & x_{0}{ }^{2}+x_{0} x_{3}+x_{3}{ }^{2}+6\left(x_{1}{ }^{2}+x_{1} x_{2}+x_{2}{ }^{2}\right), \\
F_{54}= & x_{0}{ }^{2}+x_{0} x_{3}+7 x_{3}{ }^{2}+6\left(x_{1}{ }^{2}+x_{1} x_{2}+x_{2}{ }^{2}\right), \\
F_{54}{ }^{\prime}= & \left(x_{0}+x_{1} / 2+x_{2} / 2+x_{3} / 2\right)^{2} \\
& +9\left(3 x_{1}{ }^{2}+3 x_{2}{ }^{2}+3 x_{3}{ }^{2}-2 x_{1} x_{2}-2 x_{1} x_{3}-2 x_{2} x_{3}\right) / 4,
\end{aligned}
$$

with the form-residues $(1,2,3,6),(1,3,6,18),(1,6,18,27)$, and $(1,9,9,9)$ $\bmod 3^{r} ;$ and if $p=5$,

$$
\begin{aligned}
& F_{10}^{\prime}=\left(x_{0}+x_{1} / 2+x_{2} / 2\right)^{2}+\left(11 x_{1}{ }^{2}+11 x_{2}{ }^{2}+4 x_{3}{ }^{2}+2 x_{1} x_{2}+4 x_{1} x_{3}+4 x_{2} x_{3}\right) / 4, \\
& F_{50}=\left(x_{0}+x_{1} / 2\right)^{2}+5\left(7 x_{1}{ }^{2}+4 x_{2}{ }^{2}+4 x_{3}{ }^{2}-4 x_{1} x_{2}-4 x_{1} x_{3}\right) / 4,
\end{aligned}
$$

with the residues $(1,1,5,5)$ and $(1,5,5,25), \bmod 5^{r} ;$ and if $p=11$,

$$
F_{22}=x_{0}{ }^{2}+x_{0} x_{3}+3 x_{3}{ }^{2}+2\left(x_{1}{ }^{2}+x_{1} x_{2}+3 x_{2}{ }^{2}\right),
$$

with the residue $(1,2,11,22) \bmod 11^{r}$. But all further genera so obtained contain classes of minimum not 1 .

To prove that the forms above are in genera of one class, we must correct two misprints in Smith [13, vol. II, pp. 666-668]. On page 666, $q_{3}$ in (7) should be $q_{2}$; on page $668, f_{2}$ should be $\theta_{2}$. In our (16), (17), (20), we have respectively $\left(I_{1}, I_{2}, I_{3}\right)=(1,18,1),(1,18,1),(1,6,1),(3,2,3),(3,6,3),(9,2,1)$; in all cases (working with $F_{2}$ since only modulus $2^{r}$ then matters) $f_{2}$ represents 3 $\bmod 8$, so that $I_{1} \theta_{2}$ and $I_{3} \theta_{2}$ are $3, \bmod 8$, and $\zeta=1 / 24$. Also, working with the form-residues mod $3^{r}, f_{2}$ represents respectively $1,2,2,3,6,9$, and $\theta_{2}$ represents $1,2,2,1,2,1, \bmod 3 ; \theta_{1} / 2$ and $\theta_{3} / 2$ both represent $1 \bmod 3$. Hence by Smith's formula (7),

$$
W=(1 / 12)(1 / 24) k \text {, }
$$

where (respectively for $F_{18}, F_{18}{ }^{\prime}, F_{6}{ }^{\prime}, F_{18}{ }^{\prime \prime}, F_{54}, F_{54}{ }^{\prime}$ )

$$
\begin{array}{cc}
k=2^{-1}[1-2 / 3+1 / 9] 18^{2} / 8, & 2^{-1}[1+2 / 3+1 / 9] 18^{2} / 8, \\
2^{-1}[1+2 / 3+1 / 9] 6^{2} / 8, & 4^{-1}[1+1 / 3] 27 \cdot 4 / 9, \\
8^{-1}[1-1 / 9] 27 \cdot 6^{2} / 9, & 2^{-1} \cdot 27 \cdot 4 / 9 .
\end{array}
$$

Hence $W=1 / w$, where $w=32,8,72,72,24,48$. Since $w$ is the number of positive automorphs of $F$ (cf. Theorem 8 ) in each case, these six forms are in genera of one class. The forms in (21) and (22) proceed similarly.

The existence of the following forms shows that all further genera obtained by transformations of determinant $p^{*}$ contain classes of minimum not 1: 


$$
\begin{aligned}
& 2\left(x_{0}+2^{-1} \sum x_{\alpha}\right)^{2}+\left(17 x_{1}^{2}+11 x_{2}^{2}+5 x_{3}^{2}-10 x_{1} x_{2}-4 x_{1} x_{3}-2 x_{2} x_{3}\right) / 2, \\
& 3 x_{0}^{2}+3 x_{1}^{2}+7 x_{2}^{2}+7 x_{3}^{2}-3 x_{0} x_{1}-3 x_{0} x_{2}-3 x_{0} x_{3}+3 x_{1} x_{3}+7 x_{2} x_{3}, \\
& 2 x_{0}^{2}+5 x_{1}^{2}+5 x_{2}^{2}+41 x_{3}^{2}+x_{1} x_{3}-3 x_{1} x_{2}+2 x_{0} x_{1}+2 x_{0} x_{3}, \\
& 6 x_{0}^{2}+7 x_{1}^{2}+10 x_{2}^{2}+13 x_{3}^{2}+6 x_{0} x_{1}+6 x_{0} x_{2}+6 x_{0} x_{3}+8 x_{1} x_{2}+11 x_{1} x_{3}+14 x_{2} x_{3}, \\
& 7 x_{0}^{2}+7 x_{1}^{2}+7 x_{2}{ }^{2}+9 x_{3}^{2}+3 x_{0} x_{3}-5 x_{0} x_{2}-5 x_{0} x_{1}-3 x_{2} x_{3}-3 x_{1} x_{3}-4 x_{1} x_{2}, \\
& 7 x_{0}^{2}+7 x_{1}^{2}+9 x_{2}{ }^{2}+9 x_{3}^{2}+3 x_{0} x_{3}+3 x_{0} x_{2}-5 x_{0} x_{1}+7 x_{2} x_{3}+6 x_{1} x_{3}+3 x_{1} x_{2},
\end{aligned}
$$

with respective residues $(1,2,27,54),(1,3,18,54),(1,1,81,81),(1,6,27$, $162),(1,9,9,81),(1,9,18,162), \bmod 3^{r}$;

$$
\begin{aligned}
& 2\left(x_{0}+x_{2} / 2\right)^{2}+\left(14 x_{1}^{2}+9 x_{2}^{2}+6 x_{3}^{2}+2 x_{1} x_{2}+6 x_{1} x_{3}+4 x_{2} x_{3}\right) / 2, \\
& 2\left(x_{0}+2^{-1} \sum x_{\alpha}\right)^{2}+\left(17 x_{1}^{2}+17 x_{2}^{2}+3 x_{3}^{2}-16 x_{1} x_{2}-2 x_{1} x_{3}-2 x_{2} x_{3}\right) / 2, \\
& \left(x_{0}+x_{1} / 2\right)^{2}+\left(155 x_{1}{ }^{2}+100 x_{2}{ }^{2}+20 x_{3}{ }^{2}-100 x_{1} x_{2}-20 x_{1} x_{3}\right) / 4, \\
& \left(x_{0}+x_{2} / 2+x_{3} / 2\right)^{2}+\left(260 x_{1}^{2}+35 x_{2}^{2}+35 x_{3}^{2}-20 x_{1} x_{2}-20 x_{1} x_{3}-30 x_{2} x_{3}\right) / 4,
\end{aligned}
$$

with residues $(1,1,25,25),(1,2,25,50),(1,5,25,125),(1,10,25,250)$, mod $5^{r}$, the last two forms not representing 11 and 6 respectively;

$$
3\left(x_{0}+x_{1} / 6+x_{2} / 2+x_{3} / 2\right)^{2}+\left(275 x_{1}{ }^{2}+99 x_{2}{ }^{2}+99 x_{3}{ }^{2}-66 x_{1} x_{2}-66 x_{1} x_{3}-66 x_{2} x_{3}\right) / 2 \text {, }
$$

with the residue $\left(1,11,-11,-11^{2}\right) \bmod 11^{r}$, the genera with the residues $\left(1, n, 11^{2}, n 11^{2}\right), n= \pm 1$, having been eliminated earlier.

The last step, for forms arising from $F_{2}$, is to apply transformations of determinants $3^{8}, 5^{8}$, or $11^{*}$ to the nine forms $F_{4}, F_{8}, F_{8}{ }^{\prime}, \ldots$. We shall see that $F_{4}$ yields in this way only two forms in genera of one class:

$$
F_{12}=x_{0}{ }^{2}+3 x_{3}{ }^{2}+2\left(x_{1}{ }^{2}+x_{1} x_{2}+x_{2}{ }^{2}\right), F_{36}=x_{0}{ }^{2}+3 x_{3}{ }^{2}+6\left(x_{1}{ }^{2}+x_{1} x_{2}+x_{2}{ }^{2}\right),
$$

with residues $(1,2,3,6)$ and $(1,3,6,18) \bmod 3^{r}$. The units for $F_{4}$ are \pm 1 , $\pm i_{1}, \pm i_{2}, \pm i_{3}$. If $\tau=p$, it suffices to have $p \mid 1+\lambda_{1}{ }^{2}+\lambda_{2}{ }^{2}+\lambda_{3}{ }^{2}$ with all $\lambda_{\alpha}$ prime to $p$; we have $5\left|1+1^{2}+2^{2}+2^{2}, 11\right| 1+1^{2}+2^{2}+4^{2}$. The case $\tau=p^{2}=5^{2}$ or $11^{2}$ was eliminated by our earlier treatment of $F_{2}$. To show that transformations of determinant $3^{3}$ applied to $F_{4}$ yield only (23), we need to construct forms of minimum greater than 1 with the following residues $\bmod 3^{r}:(1,1,9,9)$, $(1,2,9,18),(1,3,18,54)$, and $(1,6,18,27)$. For $(1,1,9,9)$ take $F_{18}$, replace $x_{3}$ by $2 x_{3}+x_{0}+x_{1}$, and obtain a form not representing 1 , which must be equivalent mod $2^{r}$ to $F_{4}$. For $(1,2,9,18)$ replace $x_{0}$ by $2 x_{0}-x_{1}$ in $F_{18}{ }^{\prime}$. For $(1,6,18,27)$ take $F_{54}$ and replace $x_{0}$ by $2 x_{0}$. The case $(1,3,18,54)$ was excluded even for forms derived from $F_{2}$.

We shall now see that $F_{8}$ and $F_{8}{ }^{\prime}$ yield no forms. (Since $F_{16}, F_{16}{ }^{\prime}$, and $F_{16}{ }^{\prime \prime}$ are all derivable from $F_{8}$ or $F_{8}{ }^{\prime}$ this will end the case $d=2$.) For $F_{8}$, which. has the units \pm 1 and $\pm i_{3}$ and is derived from $F_{4}$, we have only to note that $3 \mid 2+1^{2}+1^{2}+2 \cdot 1^{2}$ with $\lambda_{3}$ prime to 3 . We can write $F_{8}{ }^{\prime}$ as $\left(z_{0}{ }^{2}+z_{1}{ }^{2}+z_{2}{ }^{2}+z_{3}{ }^{2}\right) / 4$ with $z_{0}=2 y_{0}+y_{1}+y_{2}+y_{3}, z_{1}=y_{1}+y_{2}-3 y_{3}, z_{2}=y_{1}+y_{2}+y_{3}, z_{3}=y_{1}-3 y_{2}+y_{3}$. The 
units are given by $\pm\left(z_{0}, z_{1}, z_{2}, z_{3}\right)=(2,0,0,0),( \pm 1,1,1,1)$. We easily find for $p=3,5$, and 11 , solutions of $1+\lambda_{1}{ }^{2}+\lambda_{2}{ }^{2}+\lambda_{3}{ }^{2} \equiv 0 \bmod p$ not satisfying $\pm 1 \equiv \lambda_{1}+\lambda_{2}+\lambda_{3} \bmod p$. For $\tau=3^{2}$ replace $x_{0}$ and $x_{3}$ in $F_{18}$ and $F_{18}{ }^{\prime}$ by $2 x_{0}$ and $2 x_{3}$, and obtain forms equivalent $\bmod 3^{r}$ to $F_{18}$ and $F_{18}{ }^{\prime}, \bmod 2^{r}$ to $F_{8}{ }^{\prime}$.

The case $d=2$ is now complete.

We prove for $F_{3}$ that transformations of determinant $3^{s}$ give rise only to

$$
\begin{aligned}
F_{9}= & x_{0}{ }^{2}+x_{0} x_{3}+x_{3}{ }^{2}+3\left(x_{1}{ }^{2}+x_{1} x_{2}+x_{2}{ }^{2}\right), \\
F_{27}= & \left(x_{0}+x_{1} / 2+x_{2} / 2+x_{3} / 2\right)^{2} \\
& +3\left(5 x_{1}{ }^{2}+5 x_{2}{ }^{2}+5 x_{3}{ }^{2}-2 x_{1} x_{2}-2 x_{1} x_{3}-2 x_{2} x_{3}\right) / 4,
\end{aligned}
$$

with the residues $(1,3,3,9)$ and $(1,-3,9,-27)$, mod $3^{r}$. The proof, using Smith's formula, that these are in genera of one class is left to the reader. We construct the following forms of minimum greater than 1 in the genera derived from $F_{8}$ with the residues $(1,1,27,27),(1,3,9,27)$, and $(1,-3$, $-27,81) \bmod 3^{r}$ :

$$
\begin{aligned}
& 2\left(x_{0}+x_{1} / 4-x_{2} / 2\right)^{2}+\left(31 x_{1}{ }^{2}+28 x_{2}{ }^{2}+16 x_{3}{ }^{2}+4 x_{1} x_{2}+16 x_{1} x_{3}+8 x_{2} x_{3}\right) / 8 \\
& 3\left(x_{0}+x_{1} / 2+x_{2} / 2+x_{3} / 2\right)^{2}+\left(9 x_{1}{ }^{2}+13 x_{2}{ }^{2}+13 x_{3}{ }^{2}-6 x_{1} x_{2}-6 x_{1} x_{3}-10 x_{2} x_{3}\right) / 4 \\
& 7\left(x_{0}+3 x_{1} / 14-x_{2} / 7+5 x_{3} / 14\right)^{2} \\
& \quad+\left(159 x_{1}{ }^{2}+108 x_{2}{ }^{2}+87 x_{3}{ }^{2}-72 x_{1} x_{2}-30 x_{1} x_{3}-36 x_{2} x_{3}\right) / 28 .
\end{aligned}
$$

Next, applying transformations of determinant $2^{s}$ we obtain from $F_{9}$ the two forms in genera of one class

$$
\begin{aligned}
F_{18}{ }^{\prime} & =\left(x_{0}+x_{1} / 2\right)^{2}+3\left(5 x_{1}{ }^{2}+4 x_{2}{ }^{2}+4 x_{3}{ }^{2}-4 x_{1} x_{2}-4 x_{2} x_{3}\right) / 4, \\
F_{36} & =x_{0}{ }^{2}+3 x_{1}{ }^{2}+3 x_{2}{ }^{2}+9 x_{3}{ }^{2} .
\end{aligned}
$$

The genus derived from $F_{9}$ with the same residue $\bmod 2^{r}$ as $F_{12}{ }^{\prime}$ contains the form $3\left(x_{0}^{2}+x_{0} x_{1}+x_{1}^{2}\right)+4\left(x_{2}^{2}+x_{2} x_{3}+x_{3}^{2}\right)$ of minimum 2; and that with the residue $x_{0}^{2}+x_{0} x_{3}+4 x_{1} x_{2}$ mod $2^{r}$ contains the form $3\left(x_{0}+x_{2} / 2+x_{3} / 2\right)^{2}+\left(16 x_{1}{ }^{2}+13 x_{2}{ }^{2}+13 x_{3}{ }^{2}-8 x_{1} x_{2}-8 x_{1} x_{3}-10 x_{2} x_{3}\right) / 4$.

Further cases are excluded like those in the paragraph containing (18) in $\$ 13$.

The forms derived from $F_{27}$ by transformations of determinant $2:(s \geqq 1)$ are derived from that with the residue $x_{0}^{2}+x_{0} x_{3}+2 x_{1} x_{2}$ mod $2^{r}$. Such a form is the following, of minimum 4 :

$$
7\left(x_{0}+2 x_{1} / 7+2 x_{2} / 7+x_{3} / 2\right)^{2}+\left(96 x_{1}^{2}+24 x_{1} x_{2}+96 x_{2}{ }^{2}+63 x_{3}^{2}\right) / 28 .
$$

The case $d=3$ is complete.

From $F_{5}$ we get by transformations of determinant $5^{8}$ only

(28) $F_{25}=\left(x_{0}+x_{1} / 2+x_{2} / 2+x_{3} / 2\right)^{2}+5\left(3 x_{1}{ }^{2}+3 x_{2}{ }^{2}+3 x_{3}{ }^{2}+2 x_{1} x_{2}+2 x_{1} x_{3}+2 x_{2} x_{3}\right) / 4$ 
in a genus of one class, with the residue $(1,5,10,50) \bmod 5^{r}$. From $F_{25}$, the genus with the residue $x_{0}^{2}+x_{0} x_{3}+2 x_{1} x_{2} \bmod 2^{r}$ contains the form

$4\left(x_{0}+x_{1} / 4+x_{2} / 4-3 x_{3} / 8\right)^{2}+\left(60 x_{1}{ }^{2}+60 x_{2}{ }^{2}+55 x_{3}{ }^{2}-40 x_{1} x_{2}-20 x_{1} x_{3}-20 x_{2} x_{3}\right) / 16$.

We have now proved the following two theorems:

THEOREM 11. There are exactly 39 classes of positive, integral forms $f$ for which the genus of the associated norm-form $F$ contains only one class. These forms $f$ are as follows, those for which $F$ is derived from the same $F_{d}(d=2,3,5,7$, 13) being grouped together:

$\begin{array}{rlrl}d & \begin{array}{l}f=(a, b, c, r, s, t) \\ =a x^{2}+b y^{2}+c z^{2}+2 r y z+2 s z x+2 t x y\end{array} & f=(a, b, c, r, s, t) \\ 2 & (1,1,1,1 / 2,1 / 2,1 / 2) & 3 & (1,1,1,-1 / 2,0,0) \\ 4 & (1,1,1,0,0,0) & 6 & (1,1,2,-1 / 2,-1 / 2,0) \\ 6 & (1,1,2,0,0,-1 / 2) & 9 & (1,1,3,0,0,-1 / 2) \\ 8 & (1,1,2,0,0,0) & 12 & (1,1,3,0,0,0) \\ 8 & (1,1,3,1 / 2,1 / 2,1 / 2) & 12 & (1,1,4,0,0,-1 / 2) \\ 10 & (1,1,3,-1 / 2,-1 / 2,0) & 12 & (1,2,2,1 / 2,1 / 2,1 / 2) \\ 12 & (1,2,2,-1,0,0) & 18 & (2,2,2,1 / 2,1,1) \\ 16 & (1,2,2,0,0,0) & 24 & (2,2,2,0,0,-1) \\ 16 & (1,1,4,0,0,0) & 27 & (2,2,2,1 / 2,1 / 2,1 / 2) \\ 16 & (2,2,2,1,1,1) & 36 & (1,3,3,0,0,0) \\ 18 & (1,1,5,-1 / 2,-1 / 2,0) & 48 & (1,4,4,-2,0,0) \\ 18 & (1,2,3,-1,-1 / 2,0) & 5 & (1,1,2,1 / 2,1 / 2,1 / 2) \\ 18 & (1,1,6,0,0,-1 / 2) & 10 & (1,2,2,1,1 / 2,1 / 2) \\ 22 & (1,2,3,0,-1 / 2,0) & 20 & (1,2,3,-1,0,0) \\ 32 & (2,2,2,0,0,0) & 25 & (2,2,2,-1 / 2,-1 / 2,-1 / 2) \\ 32 & (2,2,3,-1,-1,0) & 7 & (1,1,2,-1 / 2,0,0) \\ 36 & (2,2,3,0,0,-1) & 28 & (1,3,3,1,1 / 2,1 / 2) \\ 50 & (2,3,3,1 / 2,1,1) & 13 & (1,2,2,-1 / 2,0,-1 / 2) \\ 54 & (2,3,3,-3 / 2,0,0) & & \\ 54 & (3,3,3,3 / 2,3 / 2,3 / 2) & & \\ 64 & (3,3,3,-1,-1,-1) & & \end{array}$

THEOREM 12. If the genus of a positive norm-form $F$ contains more than one class, then it contains at least one class not representing 1.

Our proof of this very simple result is indeed complicated. There should be some easier way of proving that if $F_{1}$ and $F_{2}$ are inequivalent norm-forms in the same genus, then there exists a third form $G$ in their genus which does not represent 1.

A description of the properties of the 39 systems, which will make them more easily accessible for applications, has been published in the Duke Mathematical Journal by Miss C. S. Williams and the author (vol. 12 (1945) pp. 527-539). 


\section{Theorem 3 is best possible.}

THEOREM 13. If the genus of a norm-form $F$ contains a class not representing 1 , then there exist infinitely many primes $p$ such that: (i) $p$ is represented by the genus of $F$, and (ii) for each $p$ there exist primitive pure quaternions $x$ (in the quaternion system associated with $F$ ) of norms divisible by $p$ but having no right-divisors of norm $p$.

We shall first prove the following lemma.

Lemma 19. If $F_{1}$ and $F_{2}$ are any two inequivalent forms in the genus of a norm-form $F$, there exist infinitely many squarefree numbers $n$, coprime in pairs, for each of which there exist integral matrices $Q$ of determinant $n^{2}$ such that $Q$ replaces $F_{1}$ by $n F_{2}$. We can suppose also that the prime factors of each $n$ are representable by the genus of $F$.

We start with the fact that there exists a transformation $T / s$, where $s$ is a positive integer which can be taken prime to any assignable number, and $T$ is an integral matrix of determinant $s^{4}$, which replaces $F_{1}$ by $F_{2}$. Hence $T$ replaces $F_{1}$ by $s^{2} F_{2}$. Taking $m=s^{2}$, we have transformations $P$ of determinant $m^{2}$ replacing $F_{1}$ by $m F_{2}$. Write $m=p^{2} r^{2}$, where $p$ is a prime not dividing $2 d$. We can factor $P$ as $Q R$, where $|Q|=p^{2}$, and $|R|=p^{2} r^{4}$ (cf. §12). Wr can suppose here that $Q$ replaces $F_{1}$ by $p F_{3}$, where $F_{3}$ is an integral form (necessarily in the genus of $F$ ).

To prove the last statement, we apply an integral transformation of determinant \pm 1 to secure $F_{1} \equiv \lambda\left(t_{0}{ }^{2}+t_{1}{ }^{2}+t_{2}{ }^{2}+t_{3}{ }^{2}\right) \bmod m^{2}$. We can thus study more easily the structure of $P$ and $Q$. Let $p^{2 s}$ be the highest power of $p$ in $m^{2}$, and let $S$ be the left factor of $P$ of determinant $p^{2 s}$. Then $S$ replaces $t_{0}{ }^{2}+t_{1}{ }^{2}+t_{2}{ }^{2}+t_{3}{ }^{2}$ by a form all of whose coefficients are divisible by $p^{8}$. By Hermite's result in $\$ 12$ we can suppose that $S$ has the form

$$
\left[\begin{array}{llll}
p^{e_{1}} & k_{1} & k_{2} & k_{3} \\
0 & p^{e_{3}} & k_{4} & k_{5} \\
0 & 0 & p^{e_{3}} & k_{6} \\
0 & 0 & 0 & p^{e_{4}}
\end{array}\right]
$$

where $2 s=e_{1}+e_{2}+e_{3}+e_{4}, 0 \leqq k_{1}<p^{e_{1}}$, and so on. Hence $p^{*}$ divides $p^{2 e_{1}}, p^{e_{1}} k_{1}$, $k_{1}^{2}+p^{2 e 2}$, and so on. From these facts we can easily deduce that $S$ has a left factor of determinant $p^{2}$, of the form

$$
Q=\left[\begin{array}{llll}
p & h & 0 & 0 \\
0 & 1 & 0 & 0 \\
0 & 0 & p & h \\
0 & 0 & 0 & 1
\end{array}\right] \text { or }\left[\begin{array}{rrrr}
p & 0 & h & k \\
0 & p & -k & h \\
0 & 0 & 1 & 0 \\
0 & 0 & 0 & 1
\end{array}\right]
$$


where $p$ divides $1+h^{2}$ or $1+h^{2}+k^{2}$. Clearly, $Q$ replaces $F_{1}$ by $p G$, where $G$ is integral.

The same argument shows that we can factor $m$ as $p_{1} p_{2} \cdots p_{0}$, where the $p_{i}$ are primes, and $P$ as $P_{1} P_{2} \cdots P_{s}$, where $\left|P_{h}\right|=p_{h}{ }^{2}$, and have $P_{1}$ replacing $F_{1}$ by $p_{1} G_{2}, P_{2}$ replacing $G_{2}$ by $p_{2} G_{3}, \cdots, P_{\text {, replacing }} G$, by $p_{s} F_{2}$. Let $G_{k}$ be the last form in this sequence which is equivalent to $F_{1}$. Then $P_{k}$ replaces $F_{1}$ by either $p_{k} F_{2}$ or $p_{k} F_{3}$, where $F_{3}$ is not equivalent to $F_{1}$. In the last case we proceed with a new transformation $P$ replacing $F_{3}$ by $m F_{2}$, with $m$ prime to $p_{k}$. We now take $G_{k}$ to be the last form in the sequence which is equivalent to either $F_{3}$ or $F_{1}$, and so introduce a new class related to $F_{1}$ either by means of a single prime $p$, or a product of distinct primes $p q$. Eventually, since the number of classes is finite, we reach $F_{2}$. Note finally that each $p$ is represented by the genus of $F$. For if (say) $F_{3}$ represents $p F_{4}$, then the solvability of $F_{4} \equiv 1$ mod $k$ implies that of $F_{3} \equiv p \bmod k$, for every modulus $k$.

Proceeding with the proof of Theorem 13, we may suppose that $F$ is carried by means of a transformation $P=\left(p_{i j}\right)$ of a determinant $m^{2}$ into $m G$, where $m$ is squarefree and prime to $2 d$, and $G$ does not represent 1 . It is now possible to find a primitive pure quaternion $x$ of norm divisible by $m$, such that the congruence system $x \bar{t} \equiv 0 \bmod m$ has the general solution $t_{i}=\sum p_{i j} z_{j}$ ( $z_{j}$ integers). We can secure $F \equiv t_{0}{ }^{2}+t_{1}{ }^{2}+t_{2}{ }^{2}+t_{3}{ }^{2}$ by a slight transformation. For each prime $p$ in $m$ we can write $P=Q R$, where $|Q|=p^{2},|R|$ is prime to $p$, and $Q$ is given by (1). The condition that $t_{i}=\sum p_{i j} z_{j}$ is equivalent for each $p$ to the condition that $Q^{-1} t$ be integral $\bmod p, t$ denoting here a column vector. We can assume that $Q$ has the second form in (1), and on taking $x_{0} \equiv 0, x_{1} \equiv 1$, $x_{2} \equiv-k, x_{3} \equiv h$, mod $p$, see that (5) of $\S 6$ is equivalent to $t=Q u$ with $u$ integral. Theorem 13 follows, since at least one factor $p$ of $m$ will have the properties stated.

It should perhaps be remarked that an $x$ cannot always be found as above if $m$ is not squarefree. For example if the rows of $\left(p_{i j}\right)$ are $(p, 0,0,0)$, $(0, p, 0,0),\left(0,0, p^{2}, k\right)$, and $(0,0,0,1)$, and $F \equiv \sum t_{i}{ }^{2} \bmod p^{4}$, then $x \bar{t} \equiv 0$ $\bmod p^{2}$ requires that $p \mid x$.

\section{REFERENCES}

1. P. Bachmann, Die Arithmetik der quadratischen Formen, I, Leipzig, 1898.

2. H. Brandt, Idealtheorie in Quaternionalgebren, Math. Ann. vol. 99 (1928) pp. 1-29.

3. L. E. Dickson, (a) Algebras and their arithmetics, Chicago, 1923; (b) Algebren und ihre Zahlenthe urie, Zürich, 1927; (c) Studies in the theory of numbers, Chicago, 1930.

4. H. Hasse, Ubber die Äquivalenz quadratischer Formen in Körper der rationalen Zahlen, Journal für Mathematik vol. 152 (1923) pp. 205-224.

5. K. Hensel, Zahlentheorie, Berlin and Leipzig, 1913.

6. C. Hermite, (a) Sur la théorie des formes quadratiques, Journal für Mathematik vol. 47 (1854) pp. 307-342; (b) vol. 41 (1850) p. 192.

7. A. Hurwitz, Vorlesungen über die Zahlentheorie der Quaternionen, Berlin, 1919.

8. B. W. Jones and G. Pall, Regular and semi-regular positive ternary quadratic forms, Acta Math. vol. 70 (1939) pp. 165-191. 
9. A. Korkine and G. Zolotareff, Sur les formes quadratiques positives quaternaires, Math. Ann. vol. 5 (1872) pp. 581-583.

10. C. G. Latimer, The classes of integral sets in a quaternion algebra, Duke Math. J. vol. 3 (1937) pp. 237-247.

11. U. V. Linnik, (a) On certain results relating to positive tenrary quadratic forms, Rec. Math. (Mat. Sbornik) N.S. vol. 5 (1939) pp. 453-471; (b) Über die Darstellung grosser Zahlen durch positive ternäre quadratische Formen, Bull. Acad. Sci. URSS Sér. Math. vol. 4 (1940) pp. 363-402.

12. G. Pall, On the factorization of generalized quaternions, Duke Math. J. vol. 4 (1938) pp. 696-704.

13. H. J. S. Smith, Collected mathematical papers, Oxford, 1894.

14. S. B. Townes, Table of reduced positive quaternary quadratic forms, Ann. of Math. vol. 41 (1940) pp. 57-58.

MCGILL UNIVERSITY, Montreal, Quebec, Canada. 